Florida International University FIU Digital Commons

FIU Electronic Theses and Dissertations

University Graduate School

$10-21-2011$

\title{
The Epidemiology of Prostate Cancer Among Multiethnic Men
}

Nicole Kellier

Florida International University, nkello01@fiu.edu

DOI: $10.25148 /$ etd.FI11120910

Follow this and additional works at: https://digitalcommons.fiu.edu/etd

\section{Recommended Citation}

Kellier, Nicole, "The Epidemiology of Prostate Cancer Among Multiethnic Men" (2011). FIU Electronic Theses and Dissertations. 534. https://digitalcommons.fiu.edu/etd/534

This work is brought to you for free and open access by the University Graduate School at FIU Digital Commons. It has been accepted for inclusion in FIU Electronic Theses and Dissertations by an authorized administrator of FIU Digital Commons. For more information, please contact dcc@fiu.edu. 


\section{FLORIDA INTERNATIONAL UNIVERSITY}

Miami, Florida

THE EPIDEMIOLOGY OF PROSTATE CANCER AMONG MULTIETHNIC MEN

A dissertation submitted in partial fulfillment of the

requirements for the degree of

DOCTOR OF PHILOSOPHY

in

PUBLIC HEALTH

by

Nicole A. Kellier

2011 
To: Interim Dean Michele Ciccazzo

R.Stempel College of Public Health and Social Work

This dissertation, written by Nicole A. Kellier, and entitled The Epidemiology of Prostate Cancer among Multicultural Men, having been approved in respect to style and intellectual content, is referred to you for judgment.

We have read this dissertation and recommend that it be approved.

Marcia Magnus

David Lee

Luther Brewster

Ahmed Albatineh

Mary Jo Trepka, Major Professor

Date of Defense: October 21, 2011

The dissertation of Nicole A. Kellier is approved.

Interim Dean Michele Ciccazzo

R.Stempel College of Public Health and Social Work

Dean Lakshmi N. Reddi

University Graduate School

Florida International University, 2011 


\section{ABSTRACT OF THE DISSERTATION \\ THE EPIDEMIOLOGY OF PROSTATE CANCER}

AMONG MULTIETHNIC MEN

Nicole A. Kellier

2011

Professor Mary Jo Trepka, Major Professor

The research goal was to document differences in the epidemiology of prostate cancer among multicultural men [non-Hispanic White (NHW), Hispanic (H), non-Hispanic Black (NHB)], and Black subgroups, particularly among NHB subgroups [US-born (USB) and Caribbean-born (CBB)]. Study findings will be useful in supporting further research into Black subgroups. Aim 1 explored changes over time in reported prostate cancer prevalence, by race/ethnicity and by birthplace (within the Black subgroups). Aim 2 investigated relationships between observed and latent variables. The analytical approaches included confirmatory factor analysis (CFA for measurement models) and structural equation modeling (SEM for regression models).

National Center for Health Statistics, National Health Interview Survey (NHIS) data from $1999-2008$ were used. The study sample included men aged 18 and older, grouped by race/ethnicity. Among the CBB group, survey respondents were limited to the English-speaking Caribbean.

Prostate cancer prevalence, by race showed a higher trend among NHB men than NHW men overall, however differences over time were not significant. CBB men reported a higher proportion of prostate cancer among cancers diagnosed than USB men overall. Due to small sample sizes, stable prostate cancer prevalence trends could not be assessed over time nor could trends in the receipt of a PSA exam among NHB men when stratified by birthplace. 
USB and CBB men differ significantly in their screening behavior. The effect of SES on PSA screening adjusted for risk factors was statistically significant while latent variable lifestyle was not. Among risk factors, family history of cancer exhibited a consistent positive effect on PSA screening for both USB and CBB men. Among the CBB men, the number of years lived in the US did not significantly affect PSA screening behavior.

When NHB men are stratified by birthplace, CBB men had a higher overall prevalence of prostate cancer diagnoses than USB men although not statistically significant. USB men were 2 to 3 times more likely to have had a PSA exam compared to CBB men, but among CBB men birthplace did not make a significant difference in screening behavior. Latent variable SES, but not lifestyle, significantly affected the likelihood of a PSA exam. 


\section{TABLE OF CONTENTS}

CHAPTER

PAGE

I. INTRODUCTION

Epidemiology of prostate cancer

Research aims and objectives

II. LITERATURE REVIEW

Prostate cancer race/ethnicity

Prostate cancer screening

Prostate cancer Risk Factors

III.

METHODOLOGY

Overview

Sample and description of data source 14

Inclusion and exclusion criteria

Variables included in the analysis

Data management and preparation 20

Statistical Analysis $\quad 22$

Aim 1: Description $\quad 23$

Aim 2: Description 26

IV. $\quad$ RESULTS 39

Overview $\quad 39$

General characteristics of the study sample $\quad 39$

Prostate cancer prevalence trends over time by race/ethnicity 47

Prostate cancer risk factors and PSA screening behaviors 56

Structural Equation Modeling : Socioeconomic Status 57

Complete mediation of the effects of exogenous variables $\quad 59$

Partial mediation of the effects of exogenous variables $\quad 62$

PSA screening and socioeconomic status within Caribbean-born 67

Complete mediation of the effects of exogenous variables 67

Structural equation model for Caribbean-born group: Partial mediation of $\quad 69$

the effects of exogenous variables

Structural Equation Modeling : Lifestyle $\quad 71$

Complete mediation of the effects of exogenous variables 74

Partial mediation of the effects of exogenous variables 76

PSA screening and Lifestyle within Caribbean-born $\quad 78$

Complete mediation of the effects of exogenous variables 78

Structural equation model for Caribbean-born group: Partial mediation of 79

the effects of exogenous variables

Partial mediation of the effects of exogenous variables : Full Model 82

Partial mediation of the effects of exogenous variables without 83

covariation between latent variables SES and lifestyle

Partial mediation of the effects of exogenous variables with covariation 88

between latent variables SES and lifestyle

Structural equation model for Caribbean-born group: Partial mediation of the effects of exogenous variables without covariation between latent 
variables SES and lifestyle

Structural equation model for Caribbean-born group: Partial mediation of the effects of exogenous variables and no covariation between latent variables lifestyle and SES

V.

DISCUSSION 100

Overview

100

Prostate cancer prevalence trends

101

Risk factors associated with prostate cancer screening behavior

103

Caribbean birthplace

107

Overall characteristics

107

Study limitations

VI. CONCLUSIONS

REFERENCES

APPENDICES 


\section{LIST OF TABLES}

TABLE

PAGE

1. Characteristics of NHIS male respondents $(1999-2008)$

2. Characteristics of male NHIS respondents ever diagnosed with cancer (all 42 types) and prostate cancer (1999-2008)

3. Characteristics of non-Hispanic Black male NHIS respondents ever diagnosed with cancer (all types) and prostate cancer (1999 - 2008)

4. Characteristics of non-Hispanic White male NHIS respondents ever diagnosed with cancer (all types) and prostate cancer (1999 - 2008)

5. Characteristics of Hispanic male NHIS ever diagnosed with cancer (all types) and prostate cancer $(1999-2008)$

6. Lifetime prevalence of cancer (all types) diagnosis: Pooled data from the 1999 -2008 NHIS

7. Lifetime prevalence of prostate cancer diagnosis: Pooled data from the $1999-$ 2008 NHIS

8. Percentage of non-Hispanic Black males who had a PSA exam by place of birth, NHIS (2005)

9. Structural equation model for predicting the probability of PSA screening as a function of the socioeconomic status latent variable

10. Structural equation model assessing the effect of SES on prostate cancer screening behavior while adjusting for birthplace, age group, family history of cancer, and years lived in the US: Complete mediation

11. Structural equation model assessing the effect of SES on prostate cancer screening behavior while adjusting for birthplace, age group, and family history of cancer: Complete mediation

12. Structural equation model for assessing the effect of SES on prostate cancer screening behavior while adjusting for birthplace, age group, family history of cancer, and years lived in the US: Partial mediation

13. Structural equation model for assessing the effect of SES on prostate cancer screening behavior while adjusting for birthplace, age group, family history of cancer, and years lived in the US: Partial mediation

14. Structural equation model of exogenous variables (family history of cancer, birthplace, and years lived in the United States), latent variable socioeconomic status, and PSA screening: Partial mediation 
15. Structural equation model assessing the effect of SES on prostate cancer screening behavior for the Caribbean-born group while adjusting for family history of cancer, age group, Caribbean birthplace, and years lived in the US: Complete mediation

16. Structural equation model assessing the effect of SES on prostate cancer screening behavior for the Caribbean-born group while adjusting for family history of cancer, age group, Caribbean birthplace, and years lived in the US: Partial mediation

17. Structural equation model for predicting the probability of PSA screening as a function of the latent variable lifestyle

18. Structural equation model assessing the effect of the lifestyle latent variable on the prostate cancer screening while adjusting for family history of cancer, age group, and birthplace: Complete mediation

19. Structural equation model assessing the effect of the lifestyle latent variable on the prostate cancer screening while adjusting for family history of cancer, age group, and birthplace: Partial mediation

20. Structural equation model assessing the effect of the latent variable lifestyle on prostate cancer screening while adjusting for family history of cancer, Caribbean birthplace, and years lived in the United States): Complete mediation

21. Structural equation model assessing the effect of the latent variable lifestyle on prostate cancer screening while adjusting for family history of cancer, age group, Caribbean birthplace, and years lived in the United States: Partial mediation

22a. Structural equation model assessing the effect of both latent variables lifestyle and SES on prostate cancer screening while adjusting for family history, age group, birthplace, and years lived in the United States

22b. Structural equation model assessing the effect of both latent variables lifestyle and SES on prostate cancer screening while adjusting for family history, age group, and birthplace

23. Structural equation model assessing the effect of both latent variables lifestyle and SES on prostate cancer screening with covariation between latent variables SES and lifestyle while adjusting for family history, age group, birthplace, and years lived in the United States

24. Structural equation model of exogenous variables (family history of cancer, age group, Caribbean birthplace, and years lived in the United States), latent variables lifestyle and socioeconomic status, and PSA screening 
25. Structural equation model assessing the effect of both latent variables lifestyle and SES on prostate cancer screening with a correlation between SES and lifestyle while adjusting for family history, age group, Caribbean birthplace, and years lived in the United States 


\section{LIST OF FIGURES}

\section{FIGURE}

PAGE

1. The probability of PSA screening as a function of the socioeconomic status latent variable

2. Prostate cancer screening as a function of the socioeconomic status latent variable adjusting for the exogenous variables: Complete mediation

3. Prostate cancer screening as a function of the socioeconomic status latent variable adjusting for the exogenous variables: Partial mediation

4. Structural equation model for predicting the probability of PSA screening as a function of the lifestyle latent variable

5. Prostate cancer screening as a function of the lifestyle latent variable, adjusting for exogenous variables: Complete mediation

6. Prostate cancer screening as a function of the lifestyle latent variable, adjusting for the exogenous variables: Partial mediation

7. Prostate cancer screening as a function of SES and lifestyle with correlation between lifestyle and SES while adjusting for exogenous variables: Full model

Percentage of men ever diagnosed with cancer (all types) by race/ethnicity, NHIS year 1999-2008

9. Age adjusted prevalence (per 100,000) of men ever diagnosed with cancer (all types), aged 45+, by race/ethnicity, NHIS year 1999-2008

10. Percentage of men ever diagnosed with prostate cancer among those diagnosed with cancer (all types) by race/ethnicity, NHIS years 1999-2008

11. Age Adjusted prevalence prostate cancer (per 1,000) by race/ethnicity and by survey year among men aged 45+, NHIS years 1999-2008

12. The probability of PSA screening as a function of the socioeconomic status latent variable

13. Prostate cancer screening as a function of the socioeconomic status latent variable adjusting for the exogenous variables: Complete mediation

14. Prostate cancer screening as a function of the socioeconomic status latent variable adjusting for the exogenous variables: Complete mediation

15. Prostate cancer screening as a function of the socioeconomic status latent variable adjusting for the exogenous variables: Partial mediation 
16. Prostate cancer screening as a function of the socioeconomic status latent variable adjusting for the exogenous variables: Partial mediation

17. Prostate cancer screening as a function of the socioeconomic status latent variable adjusting for the exogenous variables (family history, birthplace, years lived in the US: Partial mediation

18. The prostate cancer screening for Caribbean-born group as a function of the socioeconomic status latent variable adjusting for the exogenous variables:

Complete mediation

19. The prostate cancer screening as a function of the socioeconomic status latent variable for Caribbean-born group adjusting for the exogenous variables: Partial mediation

20. Structural equation model for predicting the probability of PSA screening as a function of the latent variable lifestyle

21. The prostate cancer screening as a function of the lifestyle latent variable adjusting 75 for the exogenous variables: Complete mediation

22. Prostate cancer screening as a function of the latent variable lifestyle while 76 adjusting for exogenous variables: Partial mediation

23. Structural equation model for prostate cancer screening as a function of the latent variable lifestyle for the Caribbean-born group adjusting for exogenous variables: Complete mediation

24. Structural equation model for prostate cancer screening as a function of the latent variable lifestyle for the Caribbean-born group, adjusting for the exogenous variables: Partial mediation

25a. Prostate cancer screening as a function of SES and lifestyle while adjusting for exogenous variables and assuming no covariation between SES and lifestyle

26b. Prostate cancer screening as a function of SES and lifestyle while adjusting for exogenous variables, excluding years in the US, and assuming no covariation between SES and lifestyle

26. Prostate cancer screening as a function of SES and lifestyle with correlation between lifestyle and SES while adjusting for exogenous variables

27. Prostate cancer screening as a function of latent variables SES and lifestyle while adjusting for exogenous variables among Caribbean-born men without covariation between latent variables SES and lifestyle

28. Prostate cancer screening as a function of SES and lifestyle while adjusting for exogenous variables within the Caribbean-born group with covariation between latent variables 


\section{LIST OF ACRONYMS}

\section{ACRONYM}

ACS

AIC

BIC

BMI

CAIC

CBB

CFA

CFI

DRE

FMX

GLM

$\mathrm{H}$

HHX

HMO

ML

NHB

NHIS

NHW

PPO

PSA

PSU

RMSEA

\section{DESCRIPTION}

American Cancer Society

Akaike Information Criteria

Bayesian information criterion

Body mass index

Consistent Akaike information criterion

Caribbean-born

Confirmatory factor analysis

Comparative fit index

Digital rectal examination

Family serial number

Generalized linear models

Hispanic

Household serial number

Health maintenance organization

Maximum likelihood

Non-Hispanic Black

National Health Interview Survey

Non-Hispanic White

Preferred provider organizations

Prostate specific antigen test

Primary sampling units

Root mean square error of approximation 
SEM

SES

TLI

USB
Structural equation modeling

Socioeconomic status

Tucker-Lewis index

Born in the United States 


\section{CHAPTER I.}

\section{INTRODUCTION}

\section{Epidemiology of prostate cancer}

In the United States, prostate cancer was the most common non-skin cancer among men and the second leading cause of cancer deaths among men in 2009 [1-3]. According to the American Cancer Society (ACS) approximately 240,890 men will be diagnosed with prostate cancer in 2011 (approximately 1 in 4 male cancers diagnosed annually), a higher estimate compared to 2009 , an estimate of 192,280 cases $[4,5]$. The estimated number of prostate cancer deaths for 2011 is 33,720 which is higher than the 2009 estimate of 27,360 deaths [4, 5]. From 2001 to 2005, the cancer incidence rates for all cancers among men was estimated to be 562.3 per 100,000 men and prostate cancer alone accounted for 158.2 per 100,000 men [3, 5]. Prostate cancer survival rates differ greatly by stage at diagnosis which highlights the importance of early detection. By the time a man has symptoms, the disease will have already advanced to late stage disease. Data from 1996 to 2004 indicate that the five-year survival rate for early stage diagnosis is generally $100 \%$ but drops to approximately $32 \%$ when diagnosed in late/distant stages [6].

Current established risk factors for the development of cancer overall, including prostate cancer, include overweight and obesity, low physical activity levels, high fat diet, lifestyle, and family history of cancer $[2,6]$. A significant portion (14\%-20\%) of incidence cases of cancer in the US can be attributed to overweight and obesity, and approximately $10 \%$ of prostate cancer cases can be attributed to family history of cancer [6]. The ACS emphasizes physical activity as a means of balancing food intake and energy consumption $[3,5]$. It is recommended that adults engage in 30 to 60 minutes of physical activity daily [3, 5]. 
In the United States, prostate cancer disparities exist, and there are clear differences in both incidence and mortality rates by race/ethnicity $[5,7,8]$. Prostate cancer is generally a slow growing disease and for some can have a long latency period. Prostate cancer has approximately a 100\% 5-year survival rate when diagnosed and treated in the early stages [5]. As a result of such a high 5-year survival rate, prostate cancer mortality rates should be very low. During this same time period the ACS found that among African-Americans the 5-year survival rate was 4\% lower than all other races [5]. Studies have hypothesized that this can be the result of factors such as delays in diagnosis due to low participation in prostate cancer screening, biological differences and/or environmental factors [8-10].

Over the years, cancer screening has been used as a method for reducing the probability of late-stage cancer diagnosis [5, 11-13]. The American Cancer Society currently recommends that men make an informed decision on whether or not to be screened by discussing their risk with their doctor. Men at high risk for developing prostate cancer should discuss screening with their health care provider beginning at age 45 , and men with an average risk of prostate cancer should begin having this discussion at age 50. For men who decide to be screened after consulting with their health care provider, the PSA exam is recommended which can be accompanied by the digital rectal examination (DRE). Regular participation in screening is difficult to institute, however, among all populations, particularly within minority groups and immigrant populations. There is little information available that outlines successful methods of promoting screening and increasing screening rates among minority and immigrant groups. Minority and immigrant groups are known to participate in screening activity at much lower rates $[10,14,15]$. The factors identified that may contribute to low participation rates in prostate cancer screening include: lack of access to health care, lack of education on prostate cancer and the importance of early detection, and cultural barriers to initiation and maintenance of screening 
practices $[9,10,16,17]$. Reduced access to care can be attributed to various factors including poverty $[16,18]$. Poverty determines where you live, how you pay for care, and how you physically get to and from doctor visits [18].

Factors explaining the observed disparities in incidence, mortality, and screening behaviors are not extensively studied and well understood including the role of race/ethnicity and culture $[10,17]$. Cultural identity can be used to describe a beliefs and practices of a group, which includes health behavior [19-21]. Race/ethnicity however, is used to identify a physical attributes of a group. Grouping by physical attributes lends itself to within group variation which can be defined by differences in culture. A study conducted by Gonzalez et al. (2008) found that Black men had the lowest frequency of ever having a prostate specific antigen (PSA) screening test [14]. This study population was stratified into two major groups, US-born White men, and men of African descent. Within the group of African descent, the author further stratified this cohort into 3 groups: 1) US-born black men; 2) men born in Jamaica, and; 3) men born in Trinidad and Tobago. Best-Plummer et al. (2009), analyzed the population-based cancer registry in Guyana and showed that among all cancer cases, Afro-Guyanese lead in rates of prostate, breast, and cervical cancers [22].

\section{Research aims and objectives}

The goal of this research project is to document differences in the epidemiology of prostate cancer among multicultural men (non-Hispanic White, Hispanic, non-Hispanic Black, and Black subgroups), particularly among non-Hispanic Black subgroups (US-born, foreign-born Jamaicans and other Caribbean islanders). The findings from this study will be used to support further research into Black subpopulations. The identification of varying needs within Black subpopulations can be used to create tailored interventions that serve the unique needs of each 
group. Tailored interventions and epidemiologic studies to identify disparities within Black subpopulations may raise awareness of the disease, increase screening and early detection rates, and therefore increase the probability of the reduction of morbidity and mortality rates associated with late-stage prostate cancer diagnosis among Black men. This type of information can positively impact multiethnic men in that once unique needs are identified, they can be addressed.

\section{Aim 1. To describe prostate cancer prevalence trends over time.}

Objective 1a. Describe prostate cancer prevalence trends over time by race/ethnicity (non-Hispanic White, Hispanic, non-Hispanic Black).

Objective 1b. Describe prostate cancer prevalence trends over time by non-Hispanic Black subgroups (US-born, foreign born Jamaicans and other Caribbean islanders).

Objective 1c. Document the role of ethnicity in the risk factor modeling of prostate cancer prevalence over time.

\section{Aim 2. To explore the relationship between prostate cancer risk factors and prostate cancer screening behavior.}

Objective 2a. Document the association between socioeconomic status (SES) indicators and prostate cancer screening behavior.

Objective 2b. Explore the relationship between prostate cancer screening behavior and socioeconomic status while adjusting for age group, family history of cancer, birthplace, and years lived in the United States (among those foreign born).

Objective 2c. Document the association between lifestyle indicators and prostate cancer screening behavior. 
Objective 2d. Explore the relationship between prostate screening behavior and lifestyle indicators while adjusting for age group, family history of cancer, birthplace, and years lived in the United States (among those foreign born).

Objective 2e. Explore the relationship between prostate cancer screening behavior, latent variables socioeconomic status and lifestyle, exogenous variables age group, family history of cancer, birthplace, and years lived in the United States. This objective explores the full model which incorporates all models from Objectives 2a-d. 


\section{CHAPTER II.}

\section{LITERATURE REVIEW}

\section{Prostate cancer and race/ethnicity}

It is well established that prostate cancer incidence and mortality rates are higher for African-Americans than for their White counterparts [2, 3, 23]. Not only do African-American men experience higher prostate cancer incidence, but they also present at later stages [8, 24-33]. The prostate cancer incidence rate for African-American men from 2002 to 2006, was 231.9/100,000, 1.6 times higher than the prostate cancer incidence rate for White men $(146.3 / 100,000)$ for the same period [3]. From 2002 to 2006, Hispanic/Latino men and American Indian and Alaska native men experienced incidence rates of 131.1/100,000 and 108.8/100,000 compared to $140.8 / 100,000$ and $68.2 / 100,000$ respectively for the period from 2000 to 2004 [3, 5].

Mortality rates match the trends observed with the incidence rates but with greater differences between ethnic groups. African-American men experience 1.6 times greater incidence rates than their White counterparts and approximately 2.4 times greater mortality rates with 56.3 per 100,000 deaths per year [3]. A study conducted by Fowler et al. (1999) found that even when stage of diagnosis is adjusted for, Black men with prostate cancer still experience a higher case fatality rate than do men of other races [31].

A study conducted by Glover et al. (1998), found that Jamaican men had the highest prostate cancer incidence rates $(300$ per 100,000) in the world from 1989 to 1993 [32]. It is not well understood why this group had such a high rate of prostate cancer. It is also unclear if there is an environmental influence which may impact prostate cancer risk for immigrants to the US 
who adopt a western culture. It is reported that in the United States, participation rates in screening programs among immigrant populations are lower than among native-born individuals [28]. It is not clear, however, if this is true among Caribbean born men in relation to prostate cancer screening. Taking this into account, one can postulate that as a result of less screening activity among immigrants, delays in diagnosis will be more common, resulting in a higher probability of disease progression. Undetected disease progression will then lead to a higher probability of prostate cancer mortality.

This study responds to a need for an evaluation of the epidemiology of prostate cancer among foreign born Black men who are living in the United States compared to US-born men. The proposed study is a retrospective cross-sectional study looking at prostate cancer prevalence and screening behavior among men stratified by birthplace and race/ethnicity with an emphasis on Black subgroups (namely Caribbean islanders) from 1999 to 2008. Ethnicity is defined by birthplace and will take into account cultural factors which differ by country.

Currently, all Blacks in the United States are generally categorized as African-American which does not allow for an accurate assessment of what is happening health-wise within this heterogeneous group. There are cultural differences within the group identified as AfricanAmerican as there are among Hispanics. Culture and identity greatly impact health-related beliefs, practices and risk behaviors [34-36]. Therefore, when the Black subgroups are looked at individually, differences in disease prevalence and screening behavior may emerge.

\section{Prostate cancer screening}

Although the effectiveness of prostate cancer screening is currently being debated, current diagnostic tests for prostate cancer allow the opportunity for early detection. Delays in 
diagnosis often lead to more radical and aggressive treatment choices and as a result, the likelihood of poor outcomes increases $[18,37,38]$. The cost of care increases as well when more aggressive treatment options are required. African-American participation in cancer screening (all cancer types) is reportedly lower than other racial groups [39]. Immigrants, in particular, are less likely to participate in cancer screening than any other group $[39,40]$. A study investigating differences in health seeking behavior by race/ethnicity and by birthplace found difference in the rate of pap-smears (US-born $86 \%$ vs. Foreign-born 74\%, mammogram US-born 73\% vs. 66\%, sigmoidoscopy US-born 30\% vs. Foreign-born 23\%) [41]. Low participation of immigrants in cancer screening in general can be attributed to various factors including distrust of the healthcare system, the lack of similar screening programs in their native countries and therefore the lack of awareness of current recommendations and benefits of screening [42]. Various explanations for screening disparities such as differences in socioeconomic status, access to care (which includes insurance coverage), and lack of knowledge of risks and of screening guidelines have been cited $[11,24,30,40]$. It is not well understood what factors are significant predictors of participation in prostate cancer screening among men [11].

Men at high risk of developing prostate cancer are defined by the ACS as older than age 45, African-American, and/or a positive family history of prostate cancer [3, 43]. The ACS recommends that men at high risk for the development of prostate cancer, who have at least a 10year life expectancy, and a family history of prostate cancer, begin screening at age 45 while those without a family history begin screening at age $50[6,43]$. Studies have found that men with a family history of prostate cancer tend to present with prostate cancer at earlier ages $[5,44$, 45]. A study conducted by Cotter et al. (2002), found that those men with a positive family history of at least one first degree relative diagnosed with prostate cancer had a significantly higher risk of developing prostate cancer [45]. In 2010, the American Cancer Society revised the 
prostate cancer recommendations which include incorporation of informed decision making for men who fall within the at risk guidelines (family history of prostate cancer, African-American race, and/or meet age threshold) [43]. After establishing a baseline PSA level, it is recommended that men with a PSA lower than $2.5 \mathrm{ng} / \mathrm{mL}$ screen every two years and men with a PSA $2.5-4.0$ $\mathrm{ng} / \mathrm{mL}$ screen annually and work through a risk assessment with their health care provider [43]. Further evaluation is recommended for a PSA of $4.0 \mathrm{ng} / \mathrm{mL}$ or higher.

\section{Prostate cancer risk factors}

\section{$\underline{\text { Lifestyle }}$}

As obesity and being overweight become more prevalent among the American population, lifestyle as a risk factor for prostate cancer has become increasingly important. Kushi et al. (2006) reported that approximately 3 in 10 cancer deaths annually can be attributed to lifestyle factors including diet and physical activity [7]. On the other hand, according to the ACS, approximately one third of cancer deaths annually can be attributed to lifestyle factors such as poor nutrition, lack of physical activity, and being overweight [6]. Proper nutrition guidelines to reduce the risk of developing prostate cancer are published by the ACS every 5 years [7]. The ACS generally recommends a higher daily intake of nutrient rich foods and lower daily intake of processed foods and foods high in fat [7]. Although adopting a healthy lifestyle can reduce the probability of developing cancer, it does not guarantee that a person will not develop prostate cancer in their lifetime [7]. Since culture plays a role in dietary choices and levels of physical activity each ethnic group's BMI was explored to compare ethnic group and to compare each group's trends to what is recommended to reduce the risk of developing cancer.

In the Caribbean, one in every two adults is overweight [46]. This trend can be attributed to diets high in fat and sugar and a lack of leisure physical activity in these communities [46]. As 
a means of controlling overweight and obesity, physical activity recommendations include at least thirty minutes of some form of leisure physical activity daily for at least 5 days per week $[5,7]$.

Cigarette smoking has been linked to various chronic diseases, and as of 2008, 45 million Americans were active smokers of cigarettes [6]. The use of tobacco was related to 1 in 5 deaths in the United States from 1997-2001 [6]. There is an inverse relationship between smoking status and education, the higher the education attainment the lower the prevalence of smoking [6]. The relationship between smoking and prostate cancer has not been fully elucidated. A study by Watters et al. (2009) found that men who smoked in their lifetime were less likely to be diagnosed with prostate cancer but were more likely to die from prostate cancer than men who had not smoked in their lifetime [47].

\section{$\underline{\text { Socioeconomic Status }}$}

Socioeconomic status (SES) is a well studied indicator in cancer incidence and survival for cancers such as breast, cervical and prostate cancer [48-52]. A person's SES is often classified using factors such as income level, if income their income is below the poverty line, occupation, education, and the neighborhood in which they live $[49,52,53]$. In the case of breast cancer, lower socioeconomic status is associated with lower incidence rates of breast cancer and worse survival rates. As a result, one can assume that socioeconomic status itself can be used as a predictor of cancer burden as well as screening behavior [53].

The cost for cancer diagnosis and treatment can be a significant barrier to obtaining the care necessary to ensure the best outcome for men diagnosed with prostate cancer. Out-of-pocket costs for those that are uninsured are high, and the out-of-pocket costs for those who are insured can be considered high as well due to deductibles and copayments [6]. These financial barriers 
can lead to delays in diagnosis and the receipt of treatment [6]. The high cost of screening also impacts behavior in that those with limited resources may allocate their resources to immediate needs rather than to disease prevention and/or early detection activities.

In terms of health insurance, African-Americans and Hispanics are more likely to be uninsured than non-Hispanic Whites [6]. The majority of those who are insured have employerbased health insurance. Commonly those who are working and are uninsured are low-wage workers, part-time employees, employed by small companies, or are self-employed [6]. According to the ACS, a number of private physicians will not accept new patients who are not insured by a private insurance carrier or will not accept a patient if they are not able to pay the total cost of their visit up front [6]. This practice discourages the use of non-emergency care.

In terms of education, in 2001, among non-Hispanic Whites, 25 to 64 years old, those with fewer than 12 years of education had more than twice the risk of dying from prostate cancer than those with greater than 12 years education [6]. Among African-Americans, the risk of death was 1.5 times greater for those with less than 12 years of education [6]. The results of the current study will be useful to determine whether the Caribbean-born population has the same type of relationship with educational attainment as those born in the United States. For those born in the United States, higher educational attainment is correlated with higher income. This may not be true, however, for immigrants.

Tewari et al. (2009) also explored race/ethnicity as a risk factor for prostate cancerspecific mortality controlling for clinical characteristics, demographics, and socioeconomic status [16]. This study found that Black men presented with significantly higher baseline PSA values $($ PSA $>10)$ than Whites. Although there are no distinct guidelines as to what an "abnormal" PSA 
level is, elevated PSA levels have been found to be a determinant for later stage prostate cancer diagnosis [54]. The Black men in the Tewary et al. study were found to be at increased risk of mortality related to prostate cancer. This finding is consistent with what would be expected for men with elevated PSA levels and advanced stage disease [16]. The investigators found that when assessing survival, the impact of differences in grade, stage, and comorbidities between racial groups was reduced after controlling for income and insurance status. Initial clinical characteristics such as PSA levels, stage, and grade at diagnosis as well as treatment choices were significantly different by race/ethnicity.

There are other race-related factors such as fear, worry, and emasculation concerns affecting screening behavior. A study conducted by Consedine et al. (2009), found that there are differences in fear related to cancer screening among Black subgroups [15]. This study found that differences in screening behavior within subpopulations of African descent exist. Factors such as fear and worry vary in importance by ethnic group and vary in their ability to influence screening behavior, particularly avoidance of cancer screening [15]. In addition, the study found differences in cancer-related emotional characteristics between US-born African-Americans, USborn European Americans, and English-speaking Caribbean groups. In their assessment of cancer-related fear, they found that among US-born African-Americans and US-born Europeans, cancer worry was the strongest indicator for cancer screening behavior. Among the Englishspeaking Caribbean group, however, cancer worry was the weakest indicator while emasculation concerns emerged as the strongest indicator.

In Europe, studies have been conducted that explore differences in health status and health seeking behavior among Caribbean-born individuals in comparison to others born in their host country. A study conducted by Chinegwundoh et al., found that the prostate cancer incidence rate was approximately two times greater among Caribbean-born men than for White 
men [55]. When the rates were age-adjusted, the incidence rate for Caribbean-born Black men jumped to more than three times that of White men [55]. The differences highlighted in this study show a greater disparity than what is reported among African Americans here in the United States. Here in the United States African American men are approximately 1.6 times more likely to be diagnosed with prostate cancer than Whites [3]. The authors of the study conducted by Chinegwundoh et al., recognize that the likelihood that these first generation immigrants could possibly have maintained their risk of developing prostate cancer from their country of origin as many migrated to the United Kingdom (UK) at most 50 years prior to that study period [55]. The current study sample includes years lived in the United States, unlike the study sample in the UK. 


\section{CHAPTER III.}

\section{METHODOLOGY}

\section{Overview}

This study used data from the National Center for Health Statistics, National Health Interview Survey (NHIS) from 1999 - 2008. The NHIS is a large-scale household interview survey of a statistically representative sample of the US civilian non-institutionalized population [56]. Interviewers visit 35,000 - 40,000 households across the country and collect data about $75,000-100,000$ individuals annually [56].

\section{Sample and description of data source}

The National Health Interview Survey (NHIS) is a data collection program conducted by the National Center for Health Statistics division of the Centers for Disease Control and Prevention. The NHIS is used by the Department of Health and Human Services as a tool to measure progress in achieving national health objectives by monitoring trends in health conditions such as illness and disability [56].

This cross-sectional household interview survey follows a multistage area probability sampling design. The sampling plan allows for representative sampling of households and noninstitutionalized group housing such as dormitories. The first stage of the sampling plan is taken from 1,900 geographically defined primary sampling units (PSU) in the US. This consists of a sample of 428 PSUs which are made up of one county, a group of adjacent counties, or a metropolitan statistical area[56]. Two types of second stage units are used within each PSU. A PSU includes: 1)area segments, which are defined geographically and contain as many as 16 
addresses and; 2)permit segments, which contain four addresses and use updated lists of building permits and [56].

In order to provide reliable information on minority groups the NHIS oversamples Black and Hispanic persons. The oversampling rate was as follows: 1.5:1 Blacks and 2:1 Hispanics [57]. Although NHIS samples are drawn from each state and the District of Columbia the NHIS sample is too small to provide state-level data. Therefore the data allow for estimates to be made using geographic aggregation of larger than the State level.

The NHIS collects data on timely public health needs using supplements. The main NHIS survey consists of two major parts: 1) a core questionnaire and;2) one or more sets of questions on current health topics. The core questionnaire is comprised of four major components:

1. Household survey which provides limited demographic information on all individuals in a household.

2. Family survey which collects additional demographic information on each member from each family in the household. This section collects data on health status and limitations, injuries, healthcare access and utilization, health insurance, and income and assets. From each family one sample adult and one sample child are randomly selected to be surveyed.

3. Sample adult survey which collects information on health status, health care services, and health behaviors. This survey contains information from one randomly selected adult per family.

4. Sample child survey which collects information on health status, health care services, and health behaviors. This survey contains information from one randomly selected child per family. 
In order to identify men born in the Caribbean, the variable country of birth was used. Any region or continent characterized as Latin America and the Caribbean, which includes the Central America/Caribbean region was isolated and the Central Intelligence Agency (CIA) World Factbook was used to determine the primary language for each country [58]. The CIA World Factbook is a source of information provided by the United States Central Intelligence Agency, which publishes demographic information on countries all over the world. Information available from this source includes, but is not limited to, country population, age structure, median age, ethnic groups, and languages spoken. Only countries whose primary language is listed as English were included in this analysis as the English-speaking Caribbean (Bahamas, British Virgin Islands, Cayman Islands, Dominica, Turks and Caicos, Grenada, Jamaica, St. Kitts, St. Lucia, St. Vincent, Trinidad and Tobago).

\section{Inclusion and exclusion criteria}

The study sample included men aged 18 and older who participated in the NHIS sample adult survey between $1999-2008$. The men were grouped by race/ethnicity categories which included non-Hispanic Black, non-Hispanic White, and Hispanic men. Within the non-Hispanic Black group, the men were further stratified into groups by birthplace (US-born, Caribbean-born). Non-Hispanic Black men who were born in any other country were excluded. Among the Caribbean-born group the survey respondents were limited to the English-speaking Caribbean, which include the following countries (Bahamas, British Virgin Islands, Cayman Islands, Dominica, Turks and Caicos, Grenada, Jamaica, St. Kitts, St. Lucia, St. Vincent, Trinidad and Tobago).

The NHIS sample adult survey was used for this study. The sample adult file collects information on health status, health care services, and health behaviors and includes more specific 
information collected in other surveys. The adult socio-demographics section was used to obtain information on employment. The adult file adult conditions section was used to obtain information on whether or not the men have or had cancer. The cancer diagnosis questions allow respondents to report up to three types of cancer. This increased the probability of collecting accurate information on cancer diagnosis history even if prostate cancer was not the first cancer diagnosed during the survey participant's lifetime. The conditions section also includes questions related to age at diagnosis of cancer. Those men indicating ever being diagnosed with prostate cancer were included regardless of any other cancer diagnoses. All other cancer diagnoses were excluded from this analysis. The adult behaviors section of the sample adult file contains information on smoking status and leisure physical activity. The questions on physical activity obtain details on the frequency and duration of usual leisure time physical activity.

For Aim 2, the 2005 Sample Adult file was used. In 2005, the Sample Adult file included a module on cancer control which was administered to all respondents for the sample adult questionnaire. This study utilized 5 of the 6 sections included in the cancer control module (diet and nutrition, physical activity, tobacco, cancer screening, and family history of cancer). The section on cancer screening included questions on ever receiving the prostate specific antigen test (PSA).

\section{Variables included in the analysis}

Age: The reported age was continuous. This variable was converted into a binary categorical variable as follows: 1) $<45$ years and, 2) 45 years and older.

Age of prostate cancer diagnosis: The reported age when a man was diagnosed with prostate cancer was continuous. This variable was converted into a binary categorical variable as follows: 1) $<45$ years and, 2) 45 years and older. 
Birthplace: This variable was derived from two variables: region of birth and ethnicity. The birthplace variable was designated as follows: 1) US-born and, 2) English-speaking Caribbean (Not Hispanic born in Latin America and the Caribbean).

BMI: Survey participants provided information on their height and weight which was recoded into the BMI variable. The calculated BMI value was categorized using ranges specified by the World Health Organization. The categories are as follows: underweight $(<18.5)$; healthy weight $(18.5 \leq \mathrm{BMI}<25)$; overweight, but not obese $(25 \leq \mathrm{BMI}<30)$; overweight, including obese (BMI $>=25)$; and obese $(\mathrm{BMI}>=30)$.

Caribbean birthplace: Survey participants provided information on their country of birth which was recoded as Caribbean birthplace for those born in the English-speaking Caribbean. Then each country within the Caribbean was stratified by country of birth.

Education: This variable was derived from a question on educational attainment. Anyone who reported completing high school by attaining a diploma or GED was coded as 1 . Anyone reporting not completing high school was coded as 0 .

Family history of cancer: This variable included a family history of cancer among firstdegree relatives (mother, father, sisters, and brothers). This variable was coded as follows: 1) Yes; 0) No.

FMX \& HHX: These variables were used in the process of combining datasets. FMX (family serial number) and HHX (household serial number) were used to link individual respondents' data during the appending process.

History of cancer: Survey participants were asked if they were ever told by a doctor that they had cancer. This variable was coded 0 ) never told by a doctor they had cancer; 1) yes a doctor has said I have cancer.

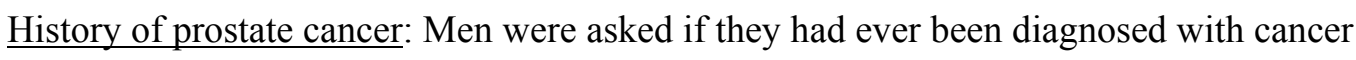
and a follow-up question on what kind of cancer. This variable was a combination of two 
variables. If respondents indicated they had not been diagnosed with cancer, then history of prostate cancer was coded as 0 ) No, not diagnosed with prostate cancer. If a respondent indicated they had been diagnosed with cancer and at least one cancer type was prostate cancer, then this variable was coded 1) Yes.

Insurance: This variable was coded 0) uninsured, self pay. The uninsured was comprised of individuals who indicated they paid for healthcare out of pocket; 1) Insured, public or private coverage. The insured group was comprise of men who indicated they were covered by a private insurer (HMO,PPO) as well as those covered by Medicare and Medicaid.

Physical activity: This variable is a combination of three questions. This variable included exercise frequency in terms of days per week, intensity, and also duration. The recommendation is moderate to vigorous physical activity at least 3 times per week. This variable was coded as follows: 1) Yes, at least 30 minutes, 3 times per week and; 0) No, less than 30 minutes, 3 times per week.

Primary Sampling Units (PSU) and strata variables: The 1995 - 2004 survey design included 358 sample PSU's [57]. State was used to form the strata for the PSUs in order to increase the probability of state level estimates for more populous states when combined with other data sources [57]. The 52 largest metropolitan areas contained one PSU each making them self-representing strata.

Prostate Specific Antigen (PSA) test: This variable was taken from the 2005 Cancer survey. This variable captured a binary response to the question "Have you ever had a PSA test (yes/no). This variable was coded 0)No, 1)Yes.

Race: Non-Hispanic White coded as 0, African-American (Black) coded as 1, and Hispanic coded as 2. Race/ethnicity included traditional (US census 2000) stratification groups. Two variables in the survey were combined to create the race/ethnicity variable for this analysis. Both race and ethnicity variables were combined to create one race/ethnicity variable. The new 
race/ethnicity variable was originally defined as follows: 1) White Non-Hispanic, 2)

Black/African American Non-Hispanic, 3) Asian, 4) Hispanic, 5) Other race, 6) Multiple race, 99) Unknown. Records with race reported as Asian, other race, and multiple races were dropped from the analysis as they were outside of the scope of the analysis. The new race/ethnicity variable was reduced to be defined as follows: 1) White Non-Hispanic, 2) Black/African American Non-Hispanic, 3) Hispanic, -99) Unknown.

Sample weight variables: In order to make inferences about the population in the US, the NHIS sample design includes national weights. The weights applied allow for design-based estimation of the US target population that is unbiased. Several years of data were pooled to conduct this analysis, and as a result, standard statistical procedures could not be used. The sampling weights provided with the dataset were adjusted as indicated by NHIS as a method of variance estimation for pooled analysis of adjacent years of data [59].

Smoking status: Smoking status was dichotomized as 1) Ever smoked, includes ever smoked during lifetime for greater than 30 days, 0) Never smoked.

Survey year: This variable was used to stratify the analysis by year.

\section{Data management and preparation}

The public use NHIS files were downloaded from the Centers for Disease Control National Center for Health Statistics website (http://www.cdc.gov/nchs/nhis/quest_data_related_1997_forward.htm). The desired variables were identified within each year and each data set was reduced to those variables needed to conduct this study. Some variables were renamed for consistency as some labels for certain questions changed over time. The files were then appended on variables. 
This study also included the use of restricted variables that were not available in the public use file. Restricted variables include geographic variables, genetic variables, temporal variables (exact dates), and detailed race/ethnicity. Caribbean birthplace was so specific that this data was included in the restricted category. As a result the study proposal was submitted for approval to access this data. Once approved, secure access files were accessed at the NCHS, NHIS office in Hyattsville, MD. This access was necessary to conduct analysis for place of birth among the Caribbean-born population. In order to protect participant confidentiality, this information is not available in the public use data files.

\section{Missing Data}

Missing data from the surveys were coded as $\mathbf{- 9 9}$. Survey questions where the study participants responded "I don't know" were coded as -99 and were treated as missing data. These missing data were not included in the analysis. If any cell during tabulations had only one person or missing information, the data was not used to calculate percentages.

In Mplus software[60], the MISSING option was used to designate data that were missing or invalid. This option allowed Mplus to use the missing data method in the analysis. Mplus provides the maximum likelihood estimation of parameters for data that are missing completely at random [60]. Missing completely at random means that missing data patterns for the predictor and outcome variables are independent of one another [61]. The data were binary, categorical, and counts. Descriptive analysis was conducted initially to identify the percentage of missing data which allowed for the assessment of the randomness of the missing data. 


\section{Statistical Analyses}

\section{$\underline{\text { STATA }}$}

Aim 1 was completed using STATA 10 statistical software. STATA allows for tabulation of frequencies, proportions, in one and two way tables. STATA is capable of completing complex statistical analysis for primary datasets as well as incorporating weights and PSUs in survey data.

The goal of Aim 1 was to explore changes over time in prostate cancer prevalence, in general, by race/ethnicity and by ethnicity within the Black subgroups. It was expected that prostate cancer prevalence profiles would differ by race/ethnicity and by ethnicity within Black subgroups. Aim 1 also intended to examine the potential correlates of the probability of prostate cancer diagnosis. The hypothesis was that correlates of prostate cancer prevalence would vary in magnitude by ethnic group over time.

\section{$\underline{\text { MPLUS }}$}

Aim 2 was completed using Mplus version 5 [60], which allows for the analysis of cross sectional and multilevel data. Mplus was used to test the theoretical Structural Equation Models. The Mplus modeling capabilities allow for models to include latent variables as well as observed variables. This software provides analysis of multiple regressions simultaneously in each specified model. It allows for modeling complex sample data and computes standard errors and chi-square tests of model fit. Since survey data incorporate weights, modeling complex sample options parameters are estimated by maximizing a weighted log-likelihood function [60].

Aim 2 analyses investigated the relationships between the observed variables as well as latent variables. The models used included confirmatory factor analysis (CFA for measurement 
models) and structural equation modeling (SEM for regression models). Measurement models state the relationships between a set of observed variables (SES and lifestyle indicators) to their respective latent variables. The structural equation model, also known as the Simultaneous Equations Model, described the relationships (pathways) between latent variables (SES and lifestyle) and observed variables (exogenous and endogenous), that are not latent factor indicators [60]. Each path or relationship is a linear regression equation for dependent numeric variables and a set of logistic regression equations for binary or ordered categorical observed dependent variables [60]. Structural equation modeling allows for more than one regression model to be run simultaneously which minimizes type one error $[60,62]$.

\section{Aim 1: Description}

Aim 1: To describe prostate cancer prevalence trends over time.

Abstraction of prostate cancer prevalence trends over time allows for the characterization of the study sample. The element of time in this analysis was survey year, which allows for the collection of prevalence data and not incidence data. The data were collected as ever diagnosed with prostate cancer and the cross section of time was when the data were collected rather than when the diagnosis occurred.

Objective 1a: Describe prostate cancer prevalence trends over time by race/ethnicity (nonHispanic White, Hispanic, non-Hispanic Black)

Prostate cancer prevalence information was extracted from the NHIS sample adult file questionnaire. Men surveyed were asked if they had ever been diagnosed with prostate cancer. The standardized age-adjusted prevalence was calculated for all reported prostate cancer diagnoses by birthplace and by race/ethnicity for survey years 1999 through 2008 . This was calculated by using the age-specific prevalence (count/age group population) $\mathrm{x}$ standard 
population (total population by race/ethnicity using year 2000 estimations) then divided by 100,000 [63-65]. This provided the age-adjusted expected prevalence per 1,000 in the population for that year.

The pattern of change in the prevalence over time was described in terms of simple polynomial trends (e.g. linear trend) and comparisons of race/ethnicity profiles were assessed. The relationship between race/ethnicity and a binary variable prostate cancer diagnosis (Y/N) was evaluated, and the results are displayed in the results section. There are several statistical assumptions that were met in order to carry out this type of analysis: existence, independence, linearity, homoscedascticity, and normality [66]. These analyses were performed using generalized linear models (GLM) approach (Poisson and/or Logistic regression models). These GLM methods allowed for determining the probability of or the assessment of race/ethnicity as a predictor of a history of prostate cancer diagnosis.

Objective 1b: Describe prostate cancer prevalence trends over time for Black subgroups

In order to assess within group variation for Black subpopulations, stratification by birthplace within the non-Hispanic Black group was performed. Black men born in the United States were compared to Caribbean-born Black men (Jamaican and other Caribbean islanders). Based on the number of men who responded yes to the question "have you ever been told by a doctor that you have prostate cancer", the men were grouped by region of birth (Caribbean, US) or by country (each Caribbean island). When stratified by country, it was found that the number of men per country was not large enough to perform meaningful analysis.

The within group variation in prostate cancer rates were modeled and assessed using generalized linear models (Poisson regression models). Linear trends over time were assessed 
and compared as described in Objective 1a. The relationship between birthplace and history of prostate cancer was evaluated. In this model, birthplace was assessed as a predictor of a history of prostate cancer diagnosis.

Objective 1c: Document the role of birthplace in the risk factor modeling of the prostate cancer prevalence over time

The planned group comparisons were the larger group (non-Hispanic Black US-born compared to non-Hispanic Black Caribbean-born) and then the subgroup analysis among nonHispanic Black Caribbean-born individuals. Subgroups were determined using birthplace which was used as a proxy to determine participant's ethnic group (Caribbean, American). To determine the probability of prostate cancer, given established risk factors in the same proportions for the non-Hispanic Black US-born versus Black subgroups (including non-Hispanic Black Caribbean-born), odds ratios were calculated as the measure of association between prostate cancer and potential risk factors (e.g. smoking status, family history of cancer, physical activity, age).

A logistic regression model was used to estimate the probability of prostate cancer diagnosis for Black subgroups adjusted for potential risk factors (smoking status, physical activity, family history of cancer, birthplace, insurance status, income, and age group) of lifetime prostate cancer diagnosis. This allowed for the assessment of risk differences between ethnic groups. To determine if the effects of each of the independent covariates were the same across each ethnic group, interaction terms (between ethnicity and each covariate) were included in the modeling process and tested for statistical significance. 


\section{Aim 2: Description}

Aim 2: To explore the relationship between prostate cancer risk factors and prostate cancer $\underline{\text { screening behavior }}$

The NHIS survey collects national survey data on each of the following variables: birthplace (US versus Caribbean born), family history of cancer (Yes/No), age, education, income, insurance, physical activity ( $\geq 30 \mathrm{~min} /$ day: Yes/No), body mass index (normal, overweight), smoking status (Yes/No), history of PSA exam (Yes/No), and years lived in the United States ( $<10$ years, $10+$ years). Two measurement models defined the two latent variables: lifestyle and socioeconomic factors. The lifestyle latent variable was defined by physical activity and smoking status as indicators. The socioeconomic latent variable was defined by income and insurance status as indicators. Finally, age, family history of cancer, years lived in the US, and birthplace were used as exogenous variables.

Confirmatory factor analysis (CFA) was used to build the measurement models related to socioeconomic status and lifestyle latent variables. This tool allowed for confirmation or rejection of the preconceived theory as it applied to this population. The theory was that lifestyle, socioeconomic status, family history of cancer, age, birthplace, and years lived in the United States (for Caribbean-born individuals) would have an impact on participation in prostate cancer screening, namely the PSA test. The path diagram illustrates the theoretical relationship between each of the indicator variables (family history of cancer, age group, birthplace, and years lived in the United States) and the latent factors (socioeconomic status and lifestyle). By running the measurement models, the CFA method used the variance and covariance structure in the data to provide parameter estimates and the corresponding standard errors, as well as all the information necessary to assess the model fit. 
The goal was to determine the relationships between variables in the model as they relate to the receipt of a PSA exam among these men. The structural equation modeling (SEM) approach was used for the assessment of relationships between multiple variables simultaneously as opposed to performing a series of multiple regression equations (logistic and linear regression) $[60,67]$. This method is powerful in that measurement error is reduced as regressions models are run simultaneously instead of several independent models [68].

Structural equations modeling is a method that uses complete data. In order to properly analyze the data and deal with missing data, the maximum likelihood (ML) estimation approach was used. The ML estimation approach consists of using available information (non-missing data) to provide a ML estimate of the sample means and covariates since the actual data are unavailable. Assumptions in using the ML estimation approach include multivariate normality, which if violated would not have a serious impact on estimates [69]. The ML estimation with robust standard errors estimation was used since indicators in this study are non-normal continuous variables. Advantages to using this approach include: 1)fewer problems with convergence; 2) under most conditions chi-square shows little bias; 3)produces the least bias under conditions of random missing data [61].

For all SEM models, the following goodness of fit indices are usually reported: a) Chisquare test along with its p-value; b) comparative fit index (CFI) and Tucker-Lewis index (TLI); c) root mean square error of approximation (RMSEA); and d) coefficient of determination or Rsquare for latent variables. The specific criteria of acceptable goodness of fit are usually: a) chisquare values that are small and non-significant; b)CFI/TLI that is $>0.95$; and c) RMSEA that is $<0.05$ [67]. However, there are other fit indices known as predictive fit indices, namely, Akaike information criterion (AIC), Consistent Akaike information criterion (CAIC) and Bayesian 
information criterion (BIC) [67] used in the SEM approach. These other methods are usually used when using the maximum likelihood estimation. They assess the model fit in a hypothetical population of replicated samples (of the same size as the sample at hand) drawn randomly from the same population. They are based on the theory of information, and the principle of minimizing the loss of information. In this sense, the smaller (close to zero), the better [67]. The AIC index was used in this study as the model fit index. The advantage of using the AIC index was that it is used as a fit index as well as an index to compare models. To test for the parsimonious model, comparisons of models (models from objectives, 2b, 2c, and 2d) were performed using the AIC index.

Univariate statistics were provided for each of the indicator and exogenous variables as well as the screening behavior variable in order to describe groups by birthplace and race/ethnicity and to compare them. The men were grouped by age $(<45,45+)$ to allow for the assessment of possible differences in screening behavior due to differences in age and more specifically recommended screening age. Proportions were calculated for the categorical variables, namely physical activity, smoking status, family history of cancer, insurance status, and education. The groups were compared in relation to physical activity once the percentage of those who participate in at least 30 minutes of leisure physical activity was calculated. Smoking status was classified as ever smoker (yes or no), and body mass index.

The homogeneity of group proportions was assessed by the Chi-square test. Comparisons of group means were performed using either t-test for independent samples, or oneway analysis of variance, or the non-parametric equivalent tests, such as, the Wilcoxon rank sum test and Kruskal-Wallis test, in case assumptions are not met to use parametric tests. Odds ratios were calculated as the measure of association between prostate cancer screening behavior and 
each of the indicators (SES and lifestyle) and exogenous variables (family history of cancer, age, race/ethnicity, years lived in the US, and birthplace).

\section{$\underline{\text { Structural Equation Model Descriptions }}$}

Objective 2a: Document the association between socioeconomic status (SES) indicators and

prostate cancer screening behavior

Figure 1. The probability of PSA screening as a function of the socioeconomic status latent variable.

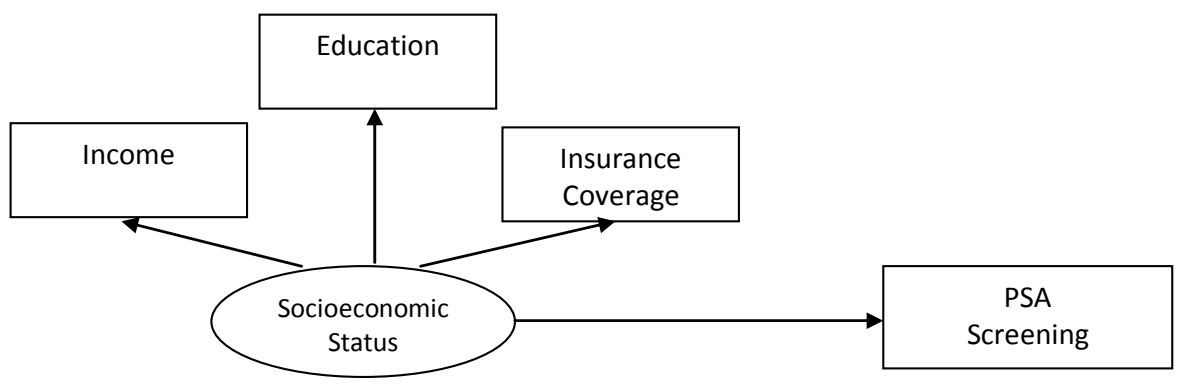

The SEM model for this objective is represented in Figure 1 above with PSA screening as the dependent variable. The latent variable focused on in this objective was socioeconomic status as defined by income, education, and insurance status (Figure 1). The SEM model is intended to predict the probability of PSA screening as a function of the SES latent variable. 
Objective 2b: Explore the relationship between prostate cancer screening behavior and $\underline{\text { socioeconomic status while adjusting for age group, family history of cancer, birthplace, and }}$ years lived in the United States (among those foreign-born)

Two SEM models are represented in Figures 2 and 3. The model in Figure 2 assessed the effect of SES on the receipt of a PSA exam while adjusting for the exogenous variables, birthplace, age group ( $<45$ years vs. $45+$ years), family history of cancer, and years lived in the United States (among those that are foreign born). The model assumed that there were no direct effects of the exogenous variables on PSA screening, that is, covariate effects impact the SES latent variable which in turn affects the probability of PSA screening.

Figure 2. Prostate cancer screening as a function of the socioeconomic status latent variable adjusting for the exogenous variables: Complete mediation.

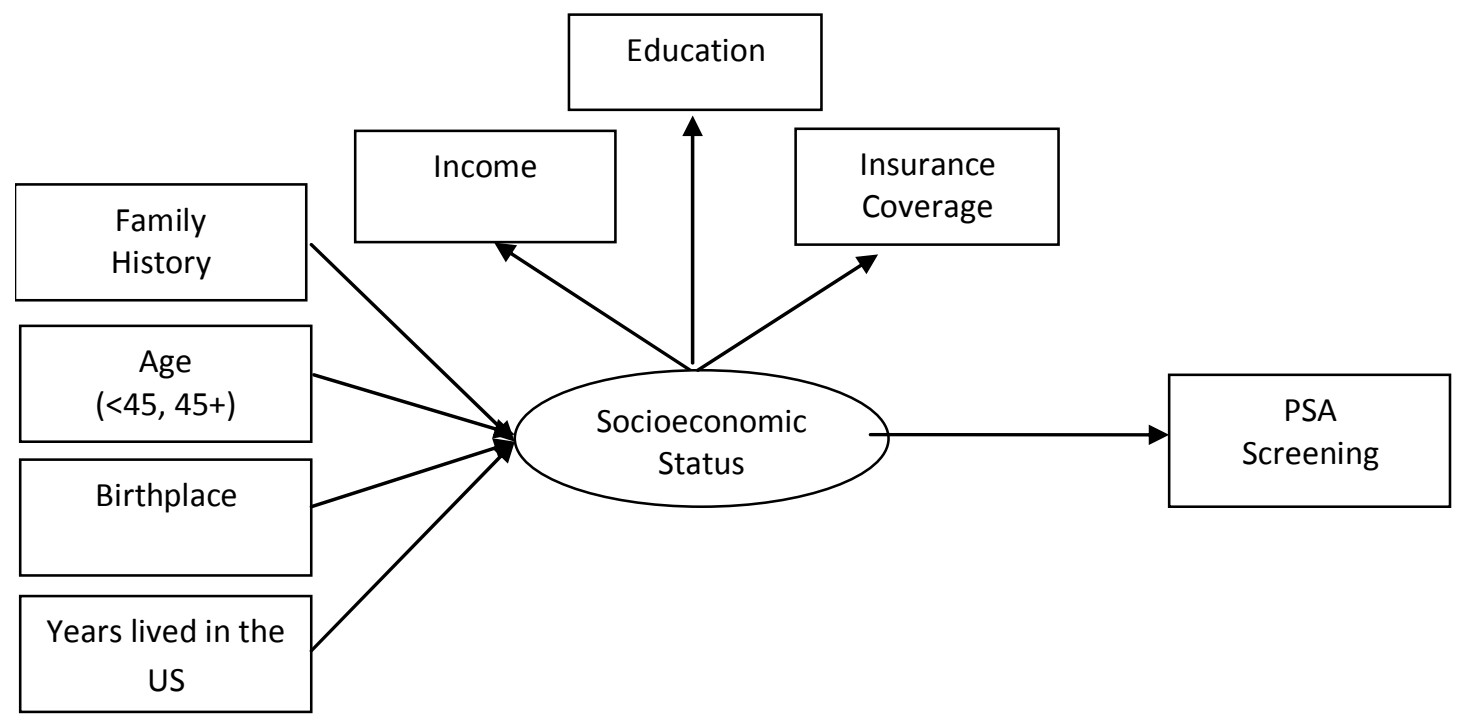


Figure 3. Prostate cancer screening as a function of the socioeconomic status latent variable adjusting for the exogenous variables: Partial mediation

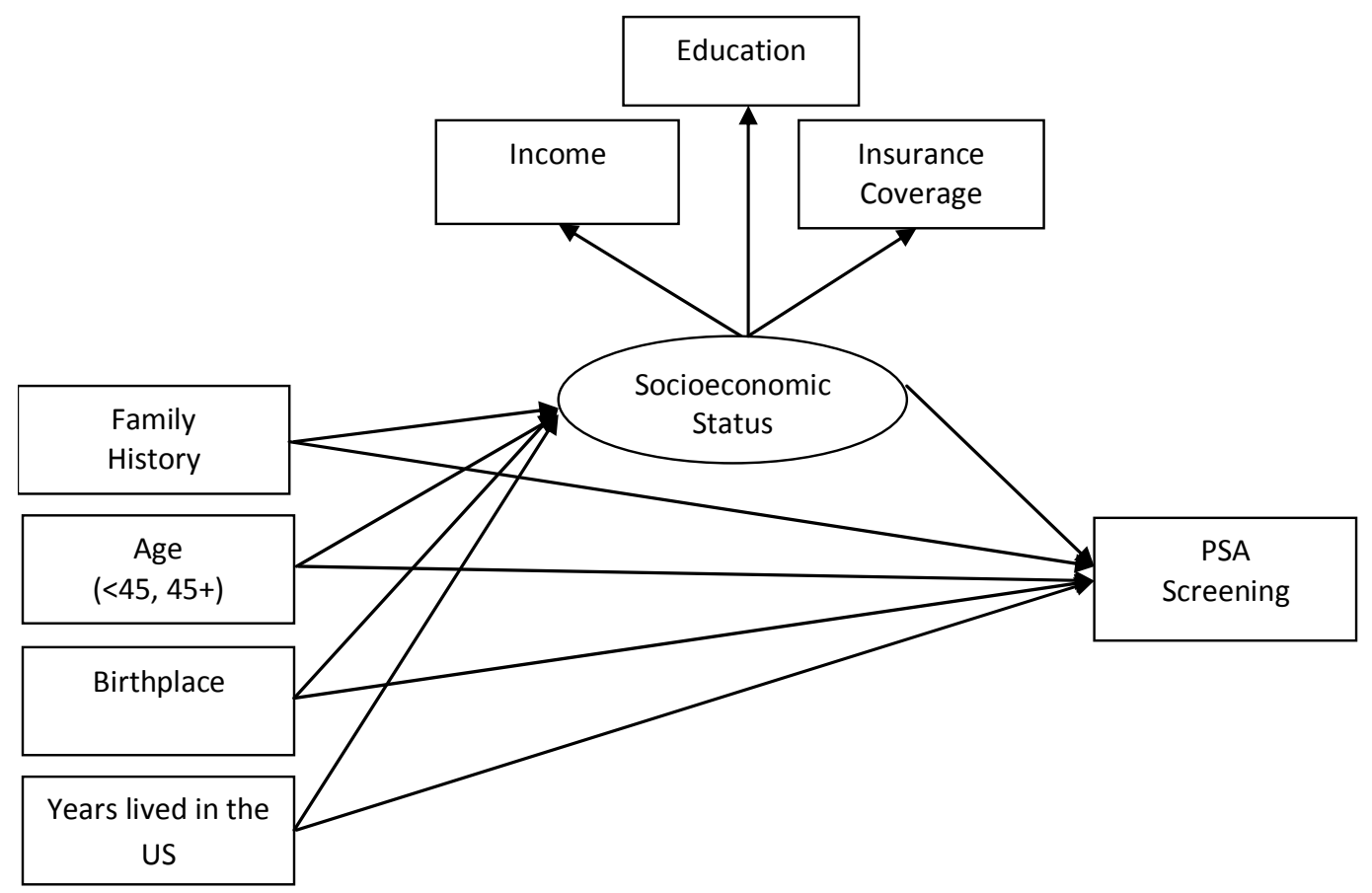

The model in figure 3 looked at the latent variable socioeconomic status as a partial mediator to exogenous variables family history of cancer, age group ( $<45$ years vs. $45+$ years $)$, birthplace (US-born vs. Caribbean-born, within Caribbean variation), and years lived in the US. This model (Figure 3) allows direct pathways (direct effects) of each exogenous variable on PSA screening behavior, as well as the indirect effects (pathways to SES latent variable). The comparison of both models was assessed by the AIC measure of model parsimony (the lower the AIC value indicates a more parsimonious model). 
Objective 2c: Document the association between lifestyle indicators and prostate cancer screening $\underline{\text { behavior }}$

In this section, the latent variable Lifestyle was evaluated to confirm the relationship with indicator variables smoking status, BMI and physical activity. This model measured the ability of the indicator to adequately define the latent factor lifestyle. Lifestyle factors generally associated with all cancers, including prostate cancer include smoking status, physical activity, and BMI. In the process of evaluating this model, if any variable was found to make the model unstable, it was removed to improve model fit. The second step was to adjust the model based on the fit indices in the original model. In this section the adjusted model evaluated latent variable lifestyle defined by indicator variables smoking status and physical activity to assess whether these two variables properly define the latent factor lifestyle.

Figure 4. Structural equation model for predicting the probability of PSA screening as a function of the lifestyle latent variable.

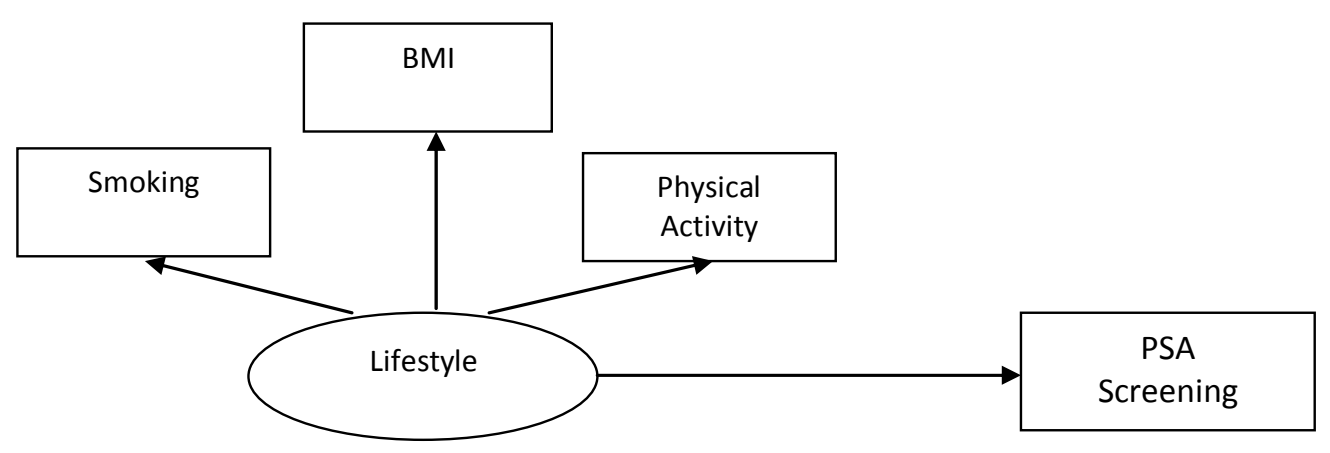


Figure 4 presents the SEM model relating PSA screening and the lifestyle latent variable.

This model assessed the affect of lifestyle on prostate cancer screening behavior.

Objective 2d: Explore the relationship between prostate cancer screening behavior and lifestyle indicators while adjusting for age group, family history of cancer, birthplace, and years lived in the United States (among those foreign-born)

Figure 5. Prostate cancer screening as a function of the lifestyle latent variable, adjusting for exogenous variables: Complete mediation

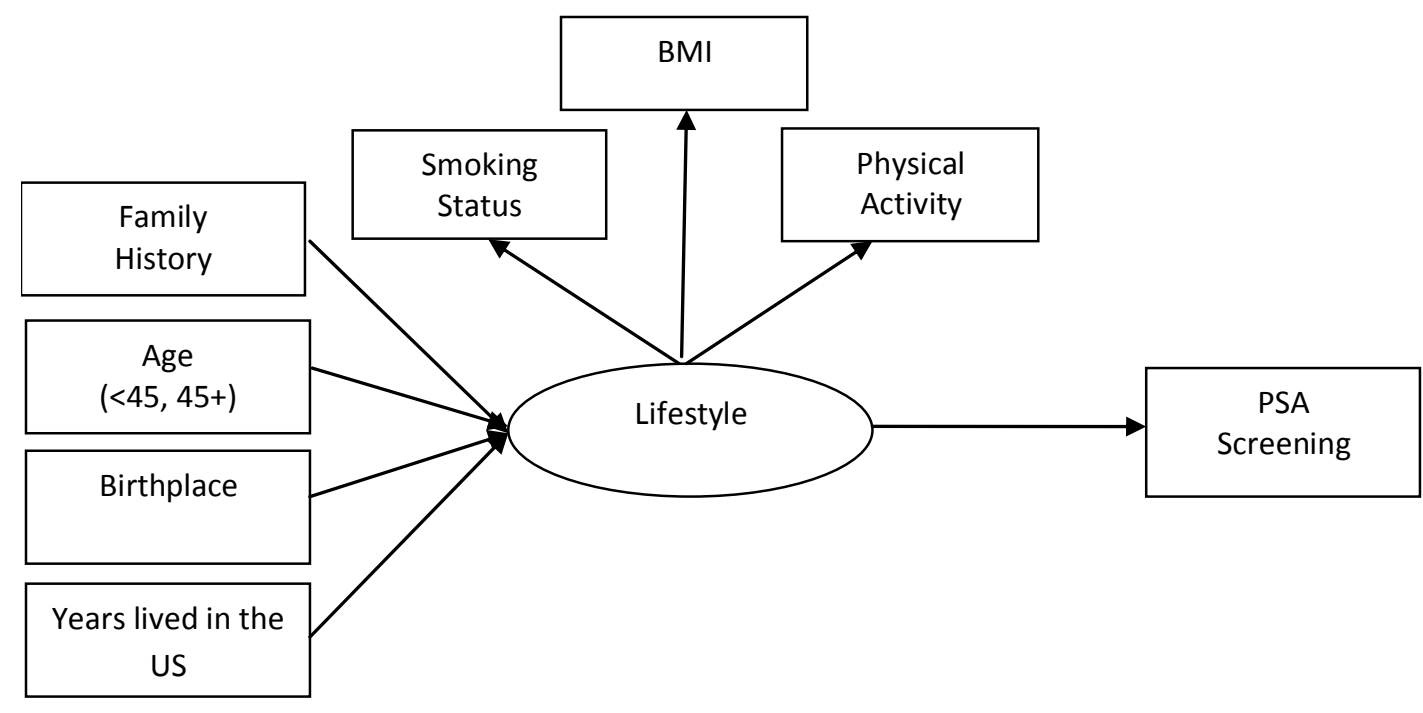

The model in Figure 5 confirms the relationship between latent variable lifestyle, defined by indicator variables smoking status and physical activity, and exogenous variables family history of cancer, age group $(<45,45+)$, birthplace, and years lived in the United States. 
The family history variable is defined by first-degree family member with a cancer diagnosis. This included history of any type of cancer for the respondent's mother, father, brother, and/or sister. Age group was defined as any man younger than age 45 versus any man in the sample aged 45 and older. This age group was chosen keeping in mind that non-Hispanic Black men are at higher risk after age 45 as opposed to age 50 for non-Hispanic White men. Birthplace is defined as born in the United States versus born in the Caribbean. Years lived in the United States is defined as born in the United States, Caribbean-born and living in the United States for less than 10 years versus Caribbean-born and living in the United States for 10 years or more. As for the lifestyle latent variable model, this model assumes that there are no direct effects of the exogenous variables on the PSA screening, that is, covariate effects impact latent variable lifestyle, which in turn affects the probability of PSA screening. 
Figure 6. Prostate cancer screening as a function of the lifestyle latent variable, adjusting for the exogenous variables: Partial mediation

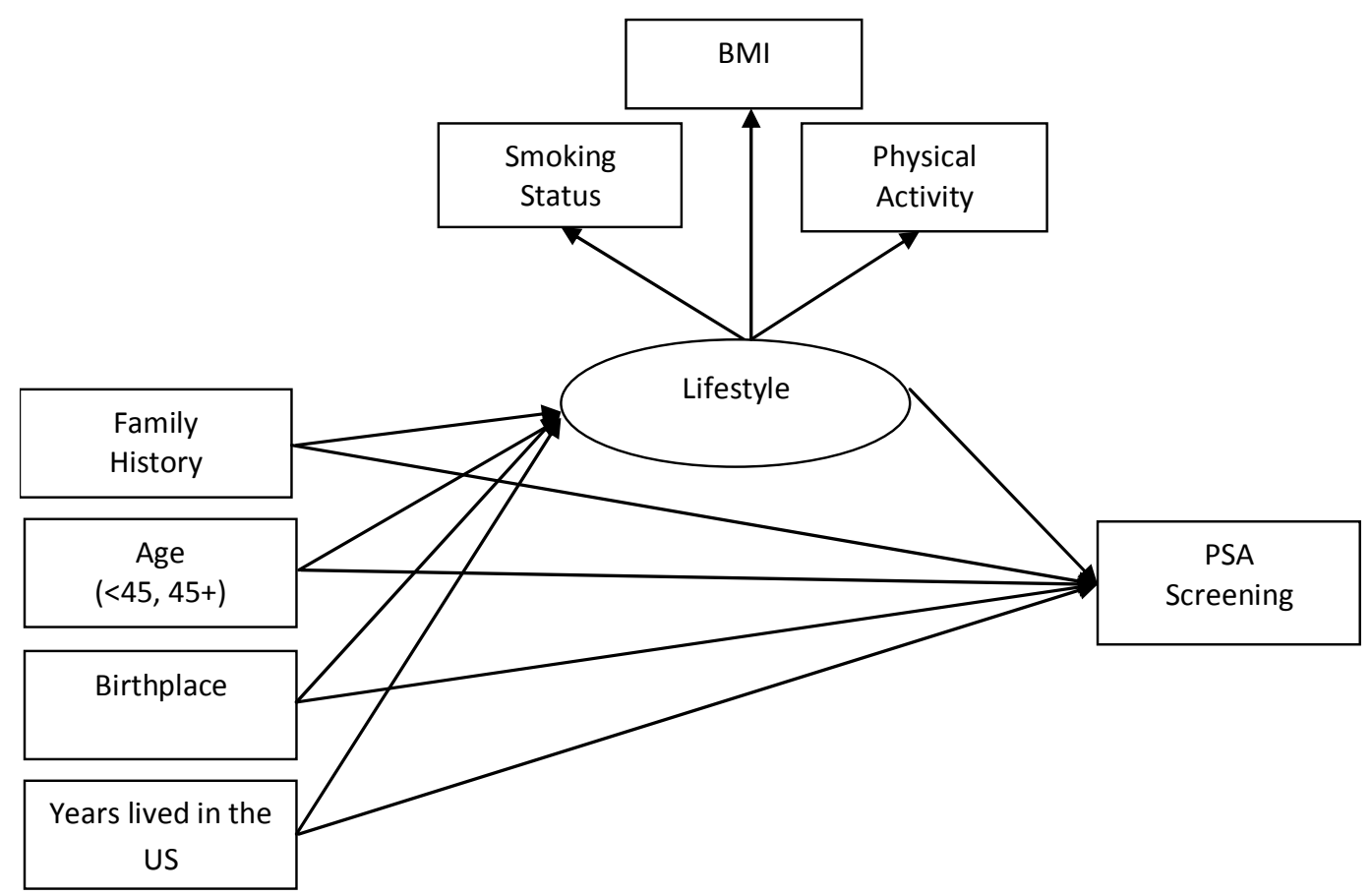

In figure 6, this model explores the relationship between the latent variable lifestyle, defined by indicator variables smoking status and physical activity, and exogenous variables family history of cancer, age group $(<45,45+)$, birthplace, and years lived in the United States. The variable birthplace takes into account differences by birthplace (US-born versus Caribbeanborn).

The SEM model was performed to assess the relationships between prostate cancer screening behavior and the lifestyle latent factor variable. It assumed lifestyle was a partial 
mediator of the relationship between exogenous variables (age, family history of cancer, race/ethnicity, and years lived in the US) and PSA screening.

Objective 2e: Explore the relationship between prostate cancer screening behavior, latent variables socioeconomic status and lifestyle, exogenous variables age group, family history of cancer, birthplace, and years lived in the United States

This objective combines objectives $2 \mathrm{a}$ through $2 \mathrm{~d}$ in order to assess the entire model (Figure 7) using both latent variables (lifestyle and socioeconomic status) while controlling for exogenous variables, family history of cancer, age group ( $<45$ years vs. $45+$ years), birthplace, and years lived in the United States. 
Figure 7. Prostate cancer screening as a function of SES and lifestyle with correlation between lifestyle and SES while adjusting for exogenous variables: Full model

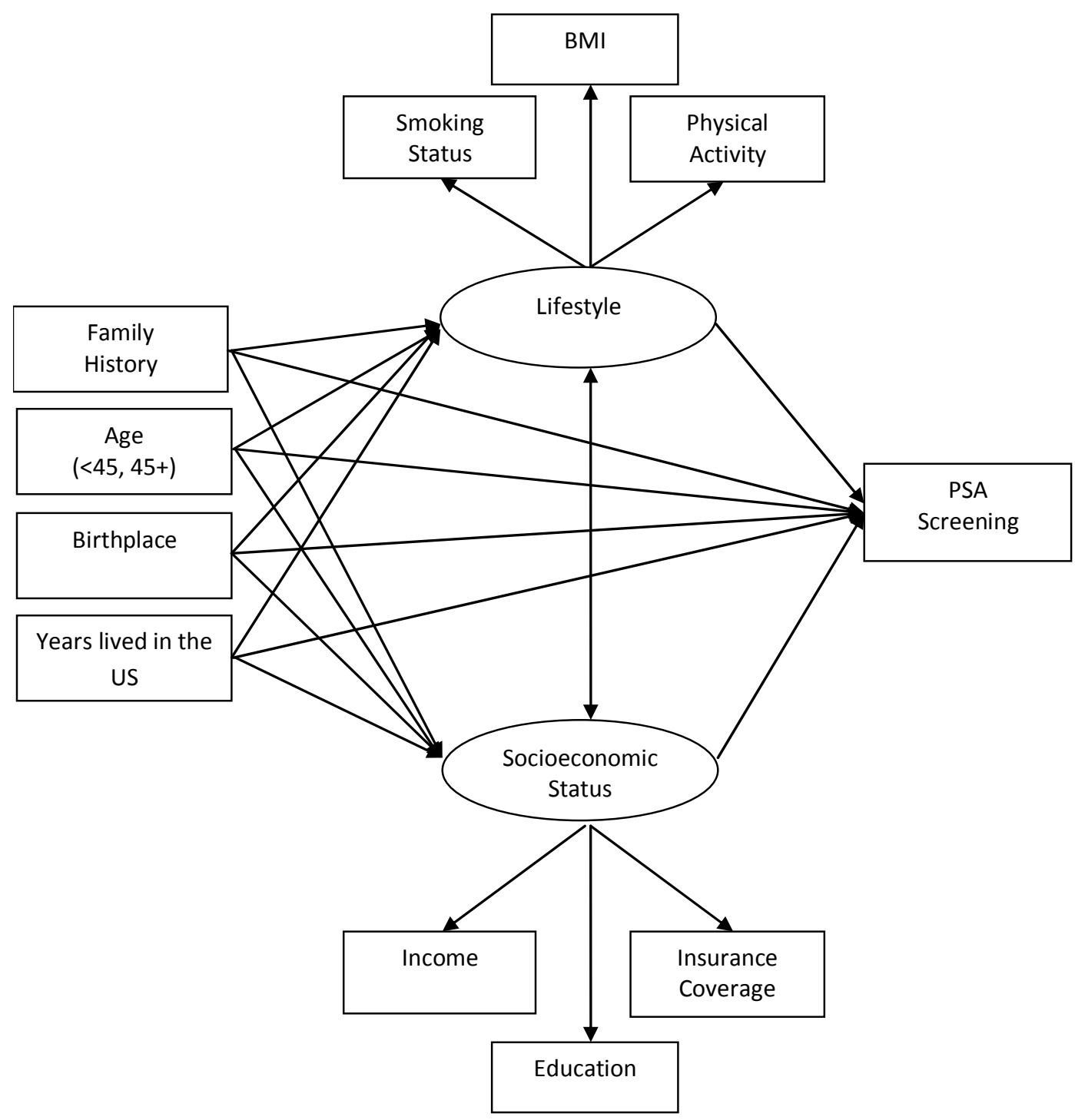


This model explored the relationships between established risk factors for prostate cancer (indicator variables) and screening behavior by conducting regression type analysis using structural equation modeling [70]. This focuses on model parsimony while evaluating the model outputs. 


\section{CHAPTER IV.}

\section{RESULTS}

\section{Overview}

All statistical analyses for the present research study were performed on a sample of adult men aged 18 and older and were conducted using weighted statistical methods. Descriptive statistics for all survey participants are shown first (Tables 1-6). The results are presented in order of each of the research aims and objectives. The main results from exploring each objective are embedded in the text below that objective.

As stated earlier, statistical analysis for Aim 1 was conducted using STATA 10 software. Statistical analysis for Aim 2 was conducted using a combination of STATA 10 software and Mplus software. In Aim 2, STATA was used to prepare the data, and Mplus was used to conduct the structural equation modeling and analysis. A summary of STATA and MPLUS commands used to conduct the analyses can be found in Tables 1a and 2a in the appendix.

\section{General characteristics of study sample}

This study sample included a total of 121,041 (unweighted sample), and over 9 million (weighted sample) men aged 18 years or older who participated in the NHIS surveys from the years 1999 through 2008. The study sample was limited to males, as the focus of this study was on prostate cancer and history of PSA test. Of these survey participants, 83,330 (72\%) were NonHispanic Whites, 15,678 (11\%) were Non-Hispanic Blacks, 21,033 (13\%) were Hispanic, and finally, $782(1 \%)$ Caribbean-born Blacks, which are a subset of the non-Hispanic Black group. 
Table 1. Characteristics of NHIS male respondents $(1999$ - 2008)

\begin{tabular}{|c|c|c|c|c|}
\hline & $\begin{array}{c}\text { Non-Hispanic } \\
\text { White(\%) }\end{array}$ & $\begin{array}{c}\text { Non-Hispanic } \\
\text { Black }(\%)\end{array}$ & $\begin{array}{c}\text { Caribbean- born } \\
\text { Black }(\%)\end{array}$ & Hispanic(\%) \\
\hline Characteristic & $\begin{array}{c}\text { Unweighted } \\
\mathrm{n}=83,330 \\
\text { Weighted } \\
\mathrm{n}=735,000,000\end{array}$ & $\begin{array}{c}\text { Unweighted } \\
\mathrm{n}=15,678 \\
\text { Weighted } \\
\mathrm{n}=108,000,000\end{array}$ & $\begin{array}{c}\text { Unweighted } \\
\mathrm{n}=782 \\
\text { Weighted } \\
\mathrm{n}=5,650,751\end{array}$ & $\begin{array}{c}\text { Unweighted } \\
\mathrm{n}=22,033 \\
\text { Weighted } \\
\mathrm{n}=130,000,000\end{array}$ \\
\hline \multicolumn{5}{|l|}{ Age group } \\
\hline$<45$ years & 48.80 & 59.52 & 56.10 & 69.93 \\
\hline $45+$ years & 51.20 & 40.48 & 43.90 & 30.07 \\
\hline Unknown & 0 & 0 & 0 & 0 \\
\hline \multicolumn{5}{|l|}{ Education } \\
\hline Some high school or less & 12.12 & 21.07 & 17.90 & 42.53 \\
\hline High school graduate & 87.02 & 77.32 & 78.18 & 55.28 \\
\hline Unknown & 0.86 & 1.61 & 3.92 & 2.19 \\
\hline \multicolumn{5}{|l|}{ Insurance } \\
\hline None, self pay & 6.91 & 12.09 & 14.24 & 24.45 \\
\hline Public or private & 86.95 & 76.84 & 71.93 & 57.79 \\
\hline Unknown & 6.14 & 11.07 & 13.83 & 17.76 \\
\hline \multicolumn{5}{|l|}{ Annual income } \\
\hline Less than 15,000 & 3.55 & 3.90 & 2.12 & 3.20 \\
\hline 15,000 or more & 49.61 & 41.67 & 50.36 & 45.17 \\
\hline Unknown & 46.84 & 54.43 & 47.52 & 51.65 \\
\hline \multicolumn{5}{|l|}{ Ever smoker } \\
\hline No & 46.38 & 55.35 & 72.60 & 60.40 \\
\hline Yes & 52.53 & 42.86 & 25.04 & 38.59 \\
\hline Unknown & 1.0 & 1.79 & 2.36 & 1.01 \\
\hline \multicolumn{5}{|l|}{ Physical activity† } \\
\hline No & 54.47 & 58.28 & 60.83 & 63.43 \\
\hline Yes & 29.80 & 25.77 & 22.73 & 21.42 \\
\hline Unknown & 15.73 & 15.95 & 16.44 & 15.15 \\
\hline \multicolumn{5}{|l|}{ Cancer diagnosis (All types) } \\
\hline No & 92.20 & 96.93 & 98.42 & 98.38 \\
\hline Yes & 7.69 & 2.99 & 1.53 & 1.58 \\
\hline Unknown & 0.11 & 0.08 & 0.05 & 0.04 \\
\hline \multicolumn{5}{|c|}{ Prostate cancer as a cancer diagnosis } \\
\hline No & 75.16 & 44.11 & 29.79 & 63.41 \\
\hline Yes & 24.44 & 55.03 & 70.21 & 36.20 \\
\hline Unknown & 0.40 & 0.86 & 0 & 0.39 \\
\hline $\begin{array}{l}\dagger \text { Physical activity is defined } \\
\text { average. }\end{array}$ & as completing at & 30 minutes of $\mathrm{pl}$ & al activity at least & nes per week on \\
\hline
\end{tabular}

Table 1 shows that among non-Hispanic White men approximately $50 \%$ are aged 45 years and older. The majority of non-Hispanic White men (87\%) have graduated from high school and almost $90 \%$ of reported being insured. Almost half of non-Hispanic White men have 
ever been smokers and less than 30\% engage in at least 30 minutes of physical activity 3 times per week. The non-Hispanic Black men in this sample are younger than the non-Hispanic White men $(\mathrm{p}<0.05)$, as approximately 60 percent are younger than 45 years old compared to approximately $48 \%$ of non-Hispanic White men in this age group. Hispanic men are the youngest group overall $(\mathrm{p}<0.05)$, with the highest proportion $(70 \%)$ of men younger than age 45 . A higher proportion of the Caribbean-born group (44\%) is aged 45+ than non-Hispanic Black men (40\%). Approximately $77 \%$ of non-Hispanic Black men (77\%) completed their high school education while approximately $87 \%$ of non-Hispanic White men and $55 \%$ of Hispanic men reported obtaining a high school education. Educational attainment for the Caribbean-born group was similar to the non-Hispanic Black subgroup (78\% and 77\% respectively). Approximately $77 \%$ of Non-Hispanic Black men reported being insured while Caribbean-born black men reported a lower proportion of coverage at $72 \%$. The group with the lowest reported proportion of being insured was among Hispanic men (24.45\%). Among Caribbean-born Black men, only 25\% reported ever being smokers while 53\% of non-Hispanic White men, $43 \%$ of non-Hispanic Black men, and $39 \%$ of Hispanic men reported ever being smokers. In terms of physical activity as recommended, $21 \%$ of Hispanic men, $23 \%$ of Caribbean-born Black men, $26 \%$ of non-Hispanic Black men, and $30 \%$ of non-Hispanic White men exercise as recommended. Non-Hispanic White men reported ever being diagnosed with cancer more often $(8 \%)$ than any other group (3\% non-Hispanic Black, $2 \%$ of Caribbean-born Black, $2 \%$ Hispanic) in this study $(\mathrm{p}<0.05)$. The other groups reported proportions of ever being diagnosed with cancer at less than $3 \%(2.99 \%$ non-Hispanic Black, 1.53\% Caribbean, 1.58 Hispanic). Among those who are diagnosed with cancer, however, more than half of non-Hispanic Black men indicate that they have been diagnosed with prostate cancer. Among Caribbean-born men approximately $70 \%$ of the reported cancer diagnoses were prostate cancer. 


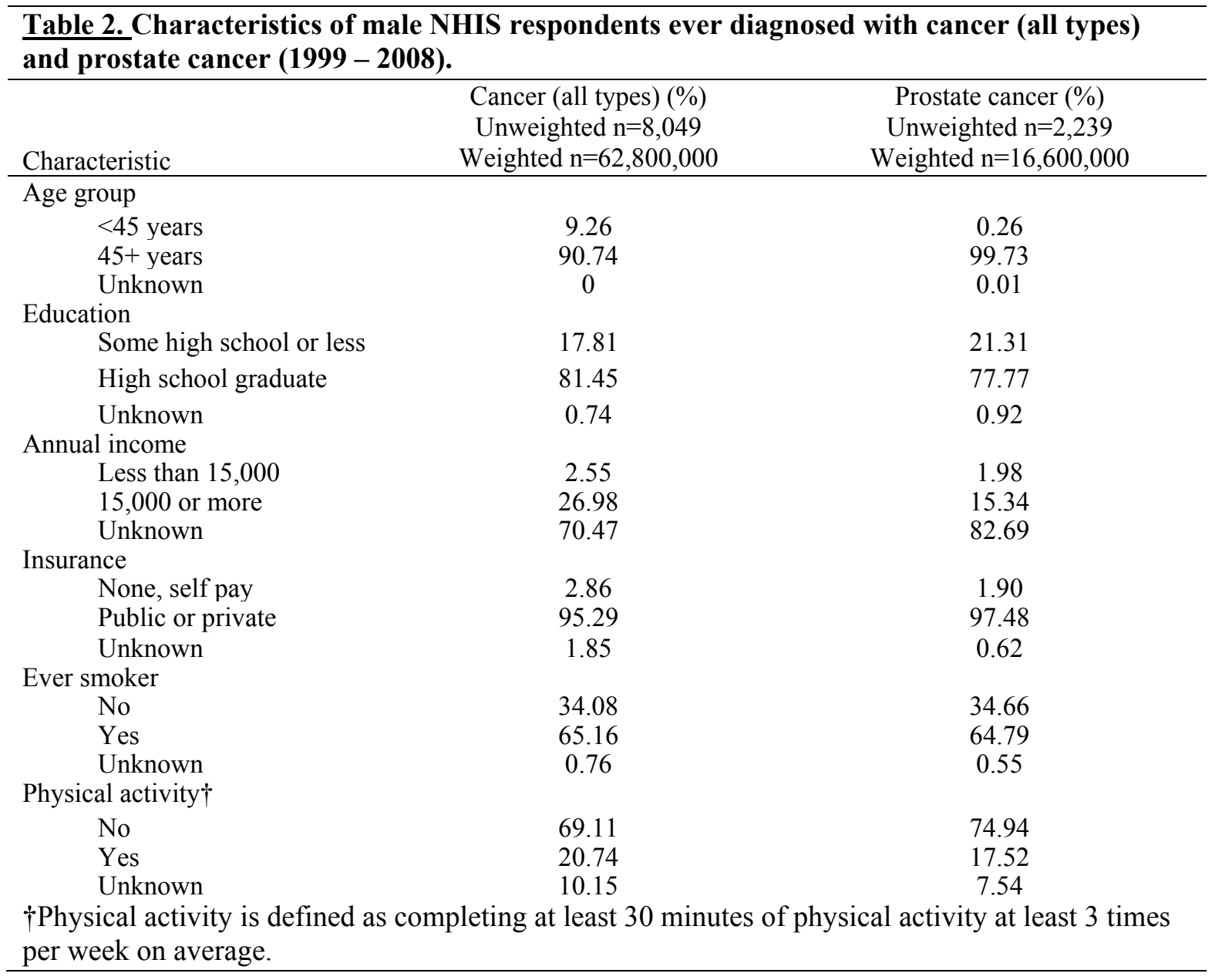

Table 2 shows the proportion of men diagnosed with cancer (all types) and more specifically prostate cancer in the 10 -year sample of men. As expected, almost all $(99.73 \%)$ prostate cancer diagnoses were reported among men older than 45 years of age. The same holds true for cancer (all types) diagnoses, although the proportion was a little lower (91\%). Almost all of the men in this sample reported being insured. A small percentage of the men diagnosed with cancer $(20.74 \%)$ exercise as often as recommended and even less $(17.52 \%)$ of the men diagnosed with prostate cancer engage in physical activity as recommended. 


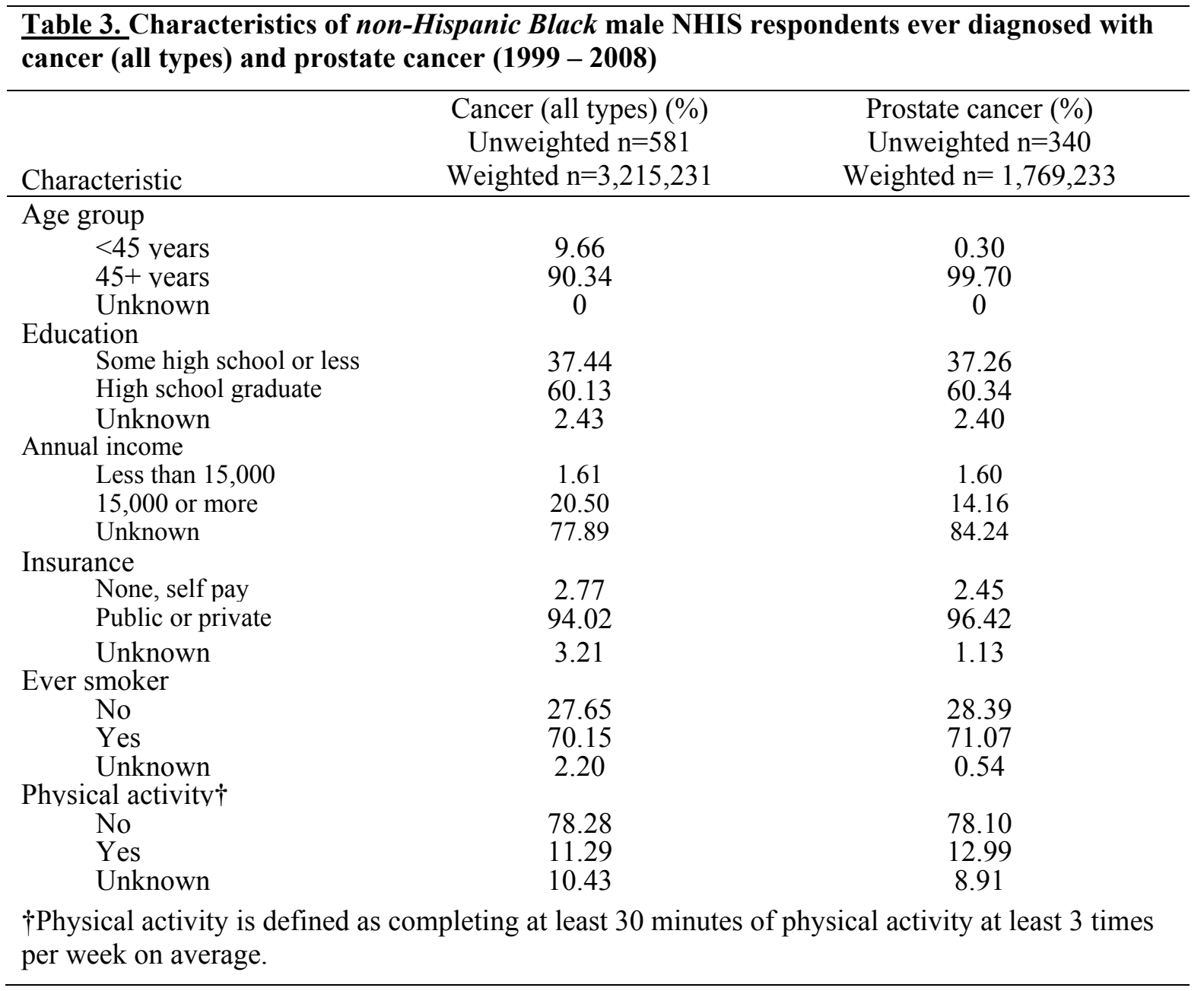

Table 3 shows the characteristics among non-Hispanic Black men diagnosed with cancer and more specifically prostate cancer. The proportion of men diagnosed with cancer (all types) and more specifically prostate cancer are very similar because many of the men diagnosed with cancer were diagnosed with prostate cancer. Within this group of non-Hispanic Black men, over $90 \%$ were 45 years and older, and almost $40 \%$ did not finish high school. A large percentage (71\%) of these men diagnosed with prostate cancer reported being ever smokers, and only $13 \%$ engaged in recommended physical activity. 


\begin{tabular}{|c|c|c|}
\hline Characteristic & $\begin{array}{c}\text { Cancer (all types) }(\%) \\
\text { Unweighted } n=6936 \\
\text { Weighted } n=56,500,000\end{array}$ & $\begin{array}{c}\text { Prostate cancer }(\%) \\
\text { Unweighted } n=1727 \\
\text { Weighted } n=13,800,000\end{array}$ \\
\hline \multicolumn{3}{|l|}{ Age group } \\
\hline$<45$ years & 9.02 & 0.28 \\
\hline $45+$ years & 90.98 & 99.72 \\
\hline Unknown & 0 & 0 \\
\hline \multicolumn{3}{|l|}{ Education } \\
\hline Some high school or less & 15.84 & 17.94 \\
\hline High school graduate & 83.58 & 81.38 \\
\hline Unknown & 0.58 & 0.68 \\
\hline \multicolumn{3}{|l|}{ Annual income } \\
\hline Less than 15,000 & 2.62 & 2.03 \\
\hline 15,000 or more & 27.45 & 15.03 \\
\hline Unknown & 69.92 & 82.94 \\
\hline \multicolumn{3}{|l|}{ Insurance } \\
\hline None, self pay & 2.79 & 1.75 \\
\hline Public or private & 95.53 & 97.75 \\
\hline Unknown & 1.68 & 0.50 \\
\hline \multicolumn{3}{|l|}{ Ever smoker } \\
\hline No & 34.09 & 34.60 \\
\hline Yes & 65.26 & 64.82 \\
\hline Unknown & 0.65 & 0.58 \\
\hline \multicolumn{3}{|l|}{ Physical activity $\dagger$} \\
\hline No & 68.30 & 74.65 \\
\hline Yes & 21.54 & 18.00 \\
\hline Unknown & 10.16 & 7.35 \\
\hline $\begin{array}{l}\dagger \text { Physical activity is defined a } \\
\text { on average. }\end{array}$ & ing at least 30 minutes of ph & ivity at least 3 times per weel \\
\hline
\end{tabular}

Table 4 shows that among non-Hispanic White men, the majority (>90\%) diagnosed with cancer (all types) and with prostate cancer were over the age of 45. As with the other racial/ethnic groups, the majority of non-Hispanic White men diagnosed with cancer were insured. This trend is the same for men diagnosed with cancer regardless of race/ethnicity. Over 80 percent of non-Hispanic White men in this sample completed high school. Approximately $65 \%$ of non-Hispanic White men reported ever being a smoker. As seen with other racial/ethnic groups, the majority of non-Hispanic White men do not engage in physical activity as recommended. 


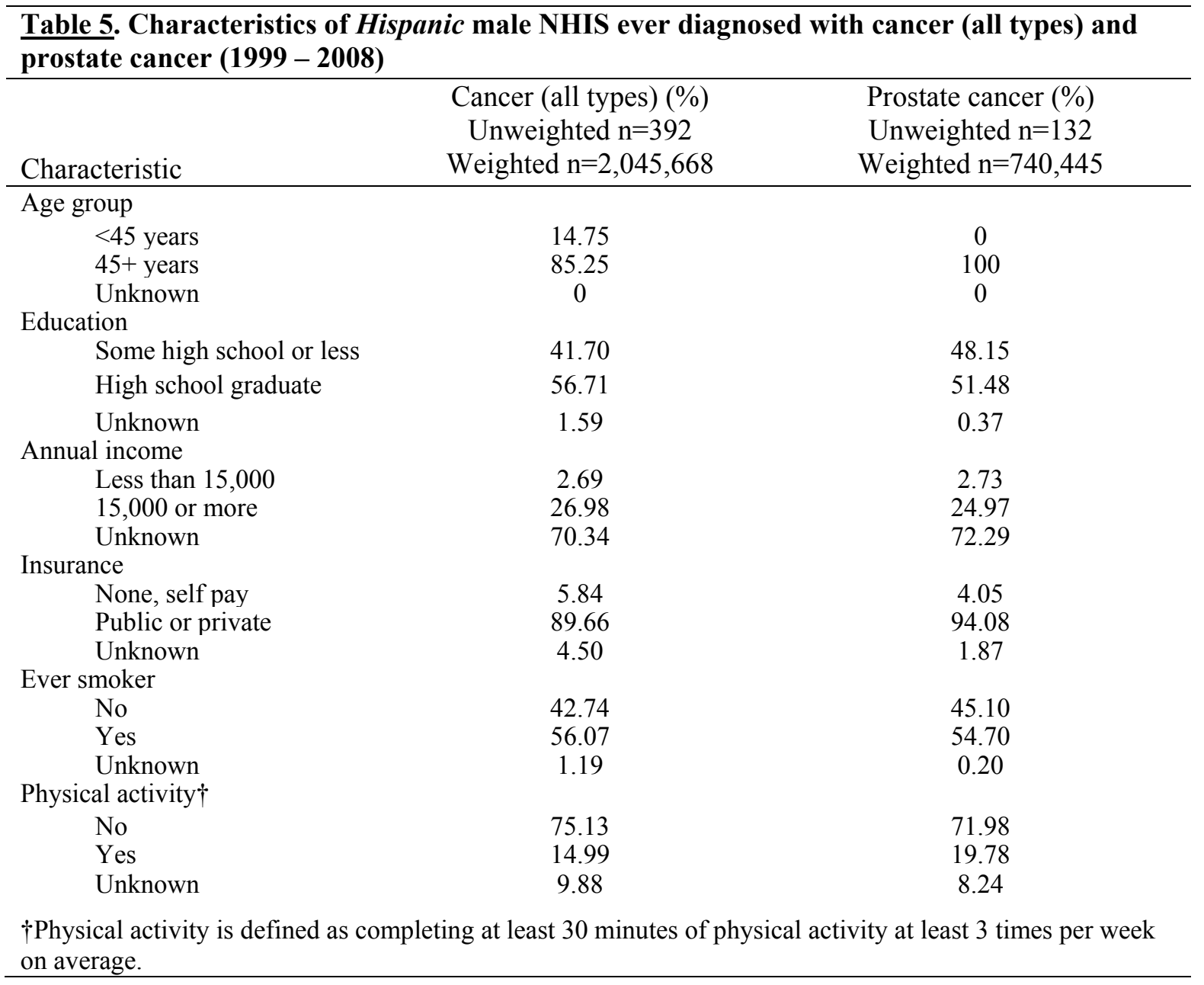

Table 5 shows that among Hispanic men, 100\% of those diagnosed with prostate cancer were aged 45 and older while only $85 \%$ of Hispanic men diagnosed with cancer (all types) were aged 45 or older. Of note, only $51 \%$ of Hispanic men reported completing high school. As with other racial/ethnic groups in this study, the majority of Hispanic men diagnosed with cancer were insured. Among the Hispanic group less that $45 \%$ reported never being smokers. Recommended levels of physical activity were reported by less than 20 percent of Hispanic men diagnosed with prostate cancer, and by less than 15 percent of those diagnosed with any cancer. 
The demographic characteristics of the Caribbean-born males in this sample are not shown due to small numbers in each category. The unweighted sample size of Caribbean born men ever diagnosed with cancer and more specifically men diagnosed with prostate cancer was 14 and 8 respectively. In order for estimates to be reliable the National Center for Health Statistics suggests not to report estimates when the number of cases is less than 50 and/or the standard error of the estimate is greater than $30 \%$ [71]. 


\section{Prostate cancer prevalence over time by race/ethnicity}

OBJECTIVE 1a: Describe prostate cancer prevalence trends over time by race/ethnicity (non-

Hispanic White, non-Hispanic Black, and Hispanic)

Figure 8. Percentage of men ever diagnosed with cancer (all types) by race/ethnicity, NHIS year 1999-2008

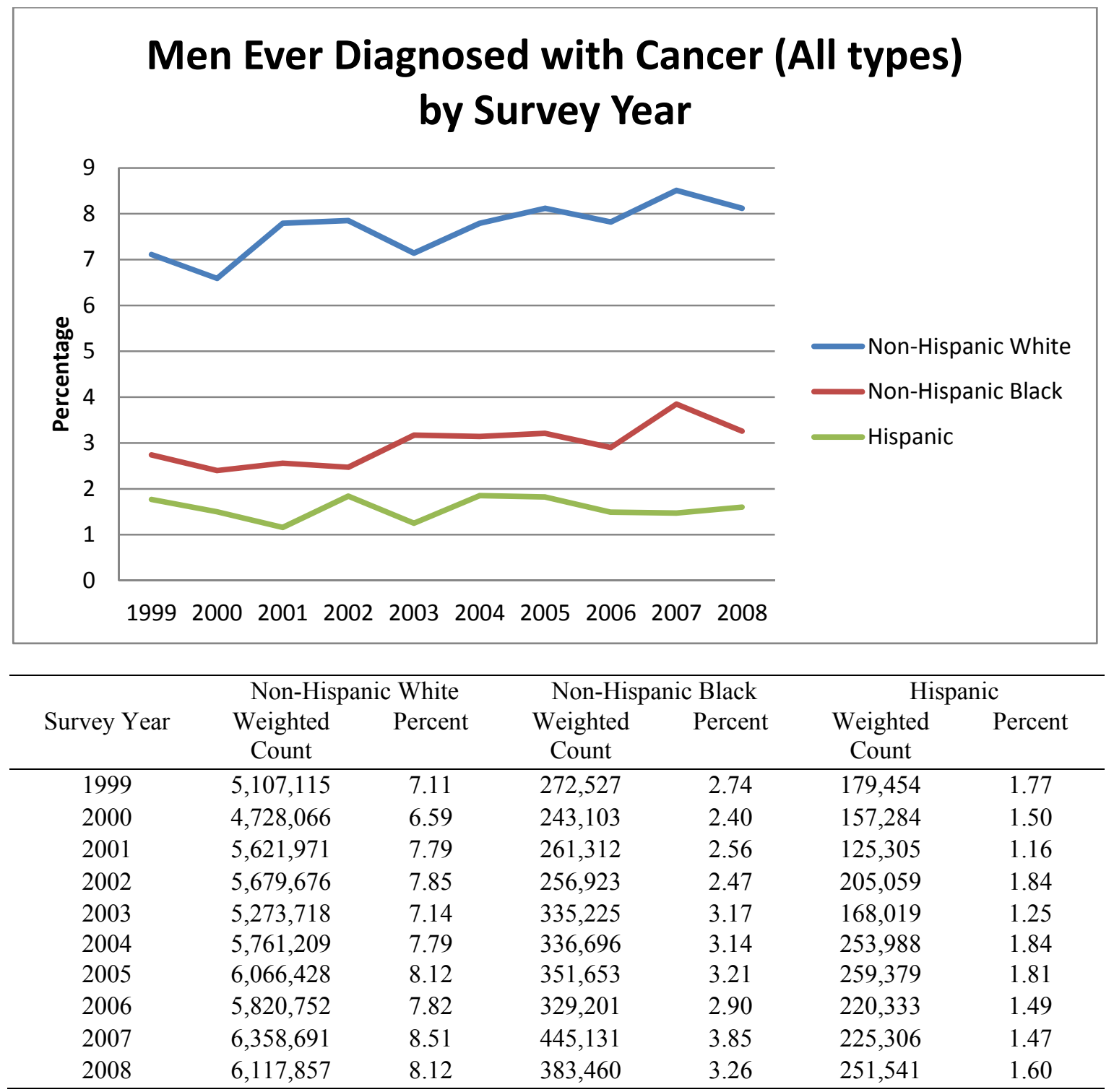


Figure 8 describes the prevalence of ever being diagnosed with cancer in survey respondents from 1999 - 2008 for all cancers. Overall non-Hispanic Whites reported ever being diagnosed with cancer, all types, more often than non-Hispanic Blacks and Hispanics. The percentage of non-Hispanic Whites indicating ever being diagnosed with cancer is 2 to 2.5 times greater than that of non-Hispanic Blacks for each year. Among non-Hispanic Whites, the percentage of responders diagnosed with cancer has risen from 1999-2008. In 1999, the percentage of non-Hispanic White respondents ever diagnosed with cancer was $7.11 \%$ and by 2008 rose to $8.12 \%$. Among non-Hispanic Black men the proportion of respondents ever diagnosed with cancer increased slightly while among Hispanic men the overall rate remained the same. Among non-Hispanic Black men the proportion overall of ever being diagnosed with cancer was between $2 \%$ and $4 \%$ during the 10 year survey period. In 2007 , the reported prevalence increased sharply from what was reported in 2006. This same trend was seen among non-Hispanic White men but not among Hispanic men. 
Figure 9. Age adjusted prevalence (per 100,000) of men ever diagnosed with cancer (all types), aged 45+, by race/ethnicity, NHIS year 1999-2008

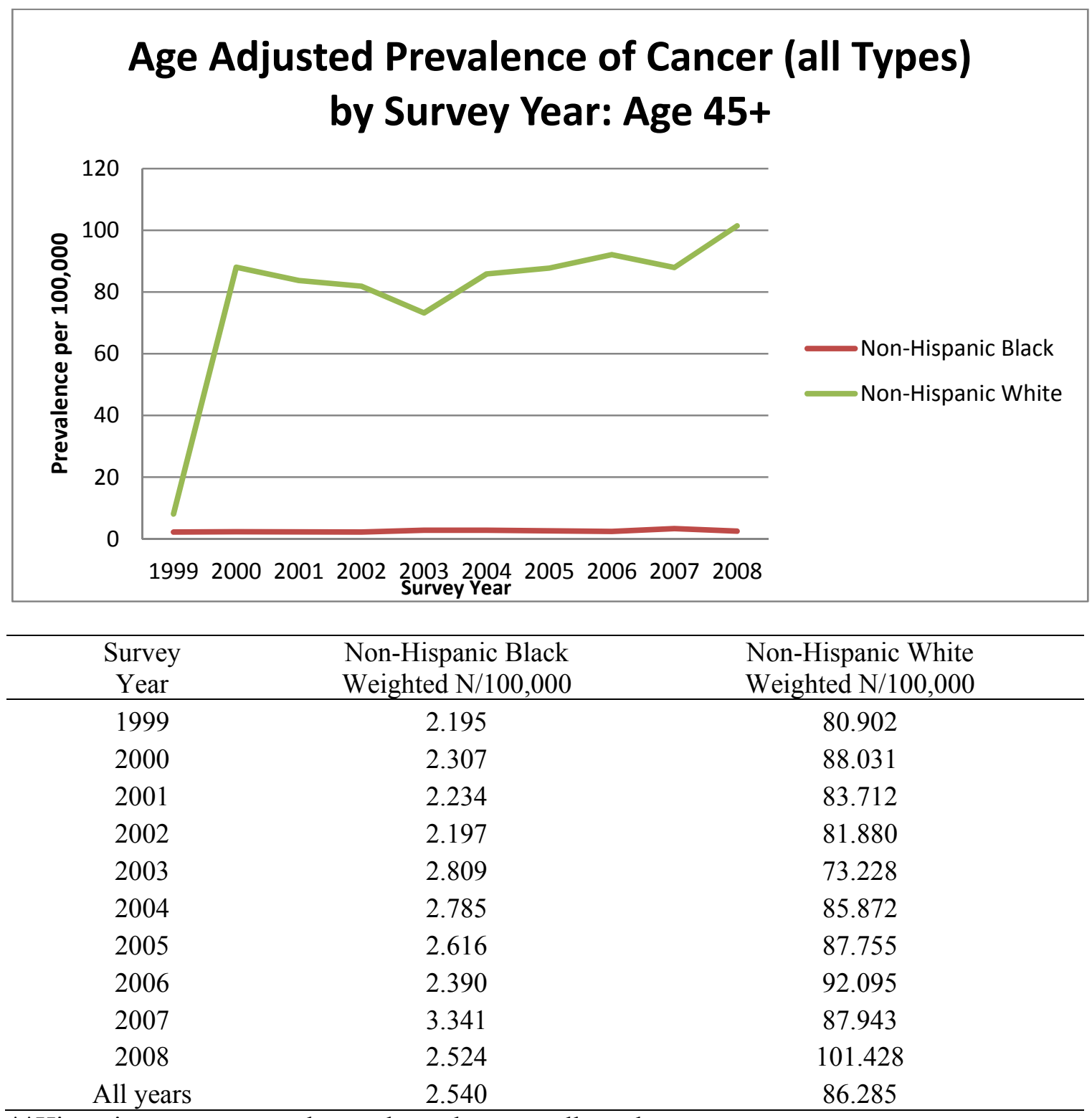

**Hispanic men were not shown above due to small numbers

The age adjusted prevalence of cancer (all types) among non-Hispanic Black men in this study sample is between 2 and 3 per 100,000 annually. In 2007, the rate was the highest (3.3/100,000). Among non-Hispanic White men the age adjusted rate of cancer was much higher. 
Among non-Hispanic White men the age adjusted rate of cancer ranged between 80 and 101 per $100,000$.

Table 6. Lifetime prevalence of cancer (all types) diagnosis: Pooled data from the 1999 2008 NHIS

\begin{tabular}{lrrr} 
& Rate ratio & p-value & $95 \%$ CI \\
\hline Survey year & 1.007 & 0.143 & $0.998,1.016$ \\
Age $(<45,45+)$ & 10 & 0.000 & $9.046,11.057$ \\
Race & & & $* * *$ \\
$\quad$ Non-Hispanic Black & 2.134 & 0.000 & $1.927,2.376$ \\
$\quad$ Non-Hispanic White & 0.659 & 0.000 & $0.564,0.771$ \\
$\quad$ Hispanic & & & \\
$* * *$ Reference group & & & \\
\hline
\end{tabular}

Poisson regression showed that there was a statistically significant difference in the overall (all years) reported diagnoses of cancer (all types) between non-Hispanic White men and non-Hispanic Black men ( $\mathrm{p}=0.000, \mathrm{CI}: 1.852,3.040)$. The difference between Hispanic men and non-Hispanic Black men however, was not significant ( $\mathrm{p}=0.121, \mathrm{CI}: 0.547,1.073)$. The differences in the rate of change by race/ethnicity over time were not significant. 
Figure 10. Percentage of men ever diagnosed with prostate cancer among those diagnosed with cancer (all types) by race/ethnicity, NHIS years 1999-2008

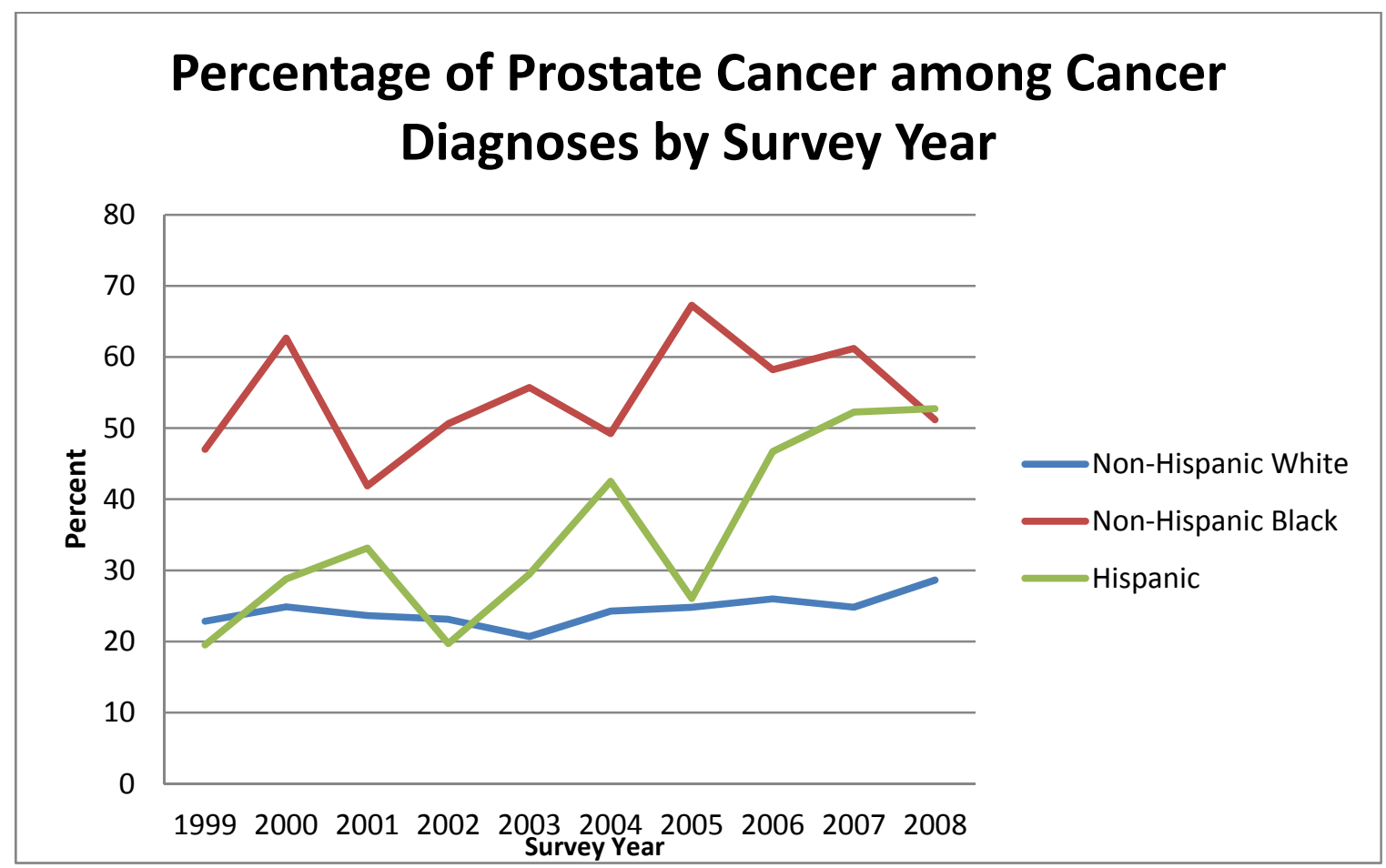

\begin{tabular}{|c|c|c|c|c|c|c|}
\hline \multirow[b]{2}{*}{ Survey Year } & \multicolumn{2}{|c|}{ Non-Hispanic White } & \multicolumn{2}{|c|}{ Non-Hispanic Black } & \multicolumn{2}{|c|}{ Hispanic } \\
\hline & $\begin{array}{c}\text { Weighted } \\
\text { Count }\end{array}$ & Percent & $\begin{array}{c}\text { Weighted } \\
\text { Count }\end{array}$ & Percent & $\begin{array}{c}\text { Weighted } \\
\text { Count }\end{array}$ & Percent \\
\hline 1999 & $1,166,605$ & 22.84 & 128,109 & 47.01 & 35,026 & 19.52 \\
\hline 2000 & $1,175,192$ & 24.86 & 152,325 & 62.66 & 45,273 & 28.78 \\
\hline 2001 & $1,328,823$ & 23.64 & 109,404 & 41.87 & 41,498 & 33.12 \\
\hline 2002 & $1,313,070$ & 23.12 & 130,030 & 50.61 & 40,393 & 19.70 \\
\hline 2003 & $1,090,389$ & 20.68 & 186,729 & 55.70 & 49,551 & 29.49 \\
\hline 2004 & $1,396,855$ & 24.25 & 165,790 & 49.24 & 107,979 & 42.51 \\
\hline 2005 & $1,503,126$ & 24.78 & 236,570 & 67.27 & 67,533 & 26.04 \\
\hline 2006 & $1,513,576$ & 26.00 & 191,660 & 58.22 & 102,882 & 46.69 \\
\hline 2007 & $1,578,910$ & 24.83 & 272,390 & 61.19 & 117,714 & 52.25 \\
\hline 2008 & $1,752,040$ & 28.64 & 196,226 & 51.17 & 132,596 & 52.71 \\
\hline
\end{tabular}

Figure 10 shows the percentage of men diagnosed with prostate cancer among those diagnosed with cancer. Among Black men who had been diagnosed with cancer, on average, approximately $55 \%$ were diagnosed with prostate cancer. In 2005 , almost $70 \%$ of non-Hispanic Black men with a cancer diagnosis indicated a past prostate cancer diagnosis which increased 
from the approximately $49 \%$ the previous year. Among Non-Hispanic White men, however, who reported a higher rate of cancer diagnosis for all cancers, approximately $24 \%$ were ever diagnosed with prostate cancer from 1999 - 2008. For each survey year the percentage of men reporting that at least one of their cancer diagnoses was prostate cancer is at least two times higher among non-Hispanic Black men than among Hispanic men. Hispanic men, indicated that among those diagnosed with cancer, approximately $36 \%$ had been diagnosed with prostate cancer. Over time, although the reported prostate cancer diagnosis percentage among Hispanic men is unstable, it is rising over time. At the beginning of the survey analysis, prostate cancer diagnoses among Hispanic men was lower than among non-Hispanic White men and in the last year of the analysis, the proportion of Hispanic men reporting prostate cancer has surpassed that of non-Hispanic Black men who had the highest rates for all other years.

For all years except for year 2008 the percentage of non-Hispanic Black men reporting positive prostate cancer diagnosis among those diagnosed with cancer show the highest proportion affected. The majority of non-Hispanic Black men, $50 \%$ or more of those diagnosed with cancer, had been diagnosed with prostate cancer. In 2005 , approximately $70 \%$ of cancer diagnoses among non-Hispanic Black men were prostate cancer. 
Figure 11. Age adjusted prevalence prostate cancer (per 1,000) by race/ethnicity and by survey year among men aged 45+, NHIS years 1999-2008

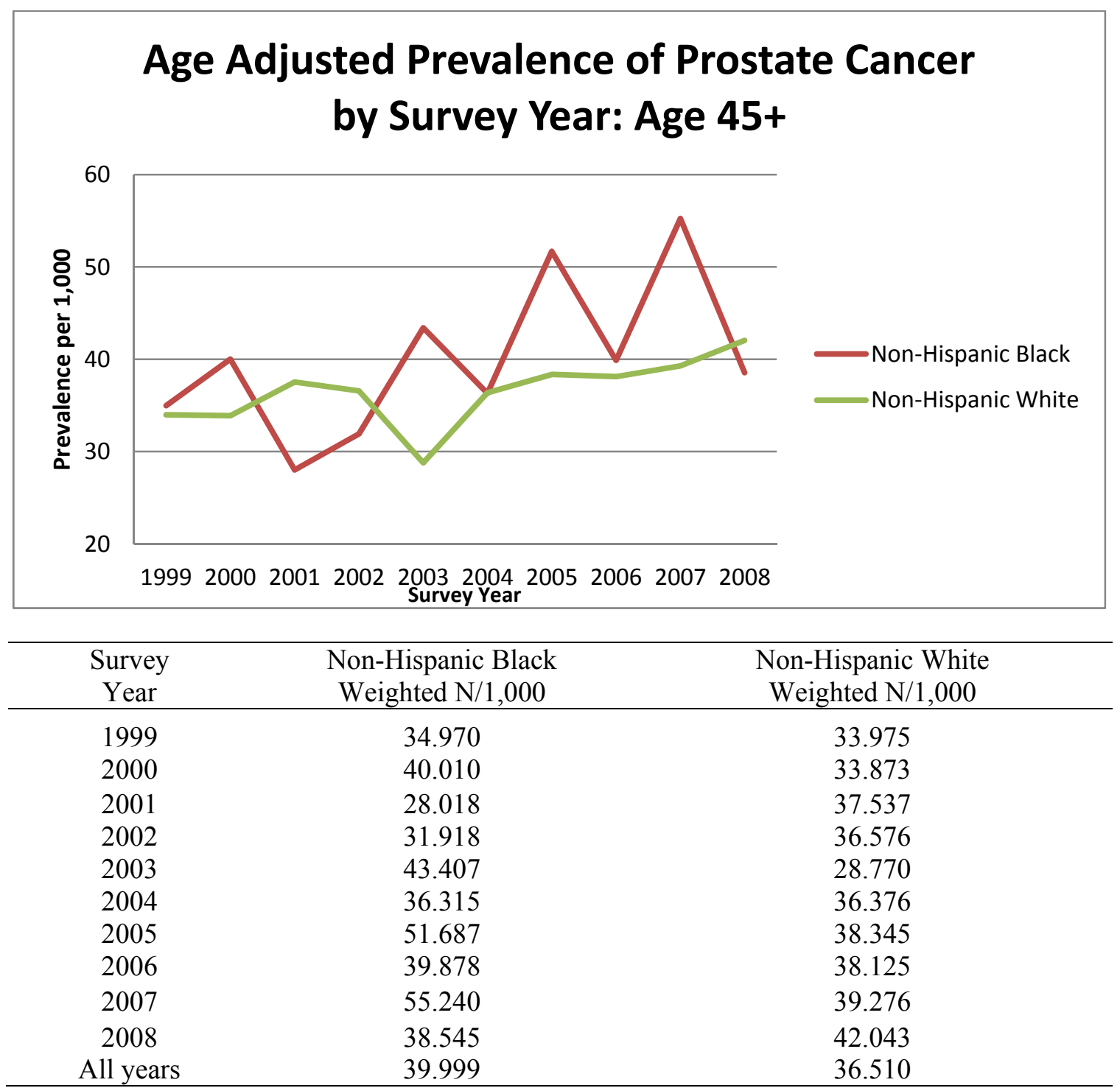

Figure 11 shows that overall the age adjusted prevalence of prostate cancer reported is higher among non-Hispanic Black men than among non-Hispanic White men over time. During survey years 2001 and 2002 the reported rate of cancer was higher among non-Hispanic White men than among non-Hispanic Black men and in the year 2004 the reported rates were found to be similar. 


\begin{tabular}{|c|c|c|c|}
\hline & Rate ratio & p-value & $95 \% \mathrm{CI}$ \\
\hline Survey year & 1.027 & 0.002 & $1.010,1.045$ \\
\hline Age $(<45,45+)$ & 394.20 & 0.000 & $116.03,1339.27$ \\
\hline \multicolumn{4}{|l|}{ Race/Ethnicity } \\
\hline Non-Hispanic Black & $* * *$ & $* * *$ & $* * *$ \\
\hline Non-Hispanic White & 0.909 & 0.183 & $0.789,1.046$ \\
\hline Hispanic & 0.464 & 0.000 & $0.355,0.606$ \\
\hline
\end{tabular}

Poisson regression showed that there was no statistically significant difference in the overall (all years) reported diagnoses of prostate cancer between Hispanic and non-Hispanic Black men ( $\mathrm{p}=0.194, \mathrm{CI}: 0.99,1089)$. The reported rate of prostate cancer was not significantly different between non-Hispanic White men and non-Hispanic Black men overall (all years) $(p=0.990, C I: 0.728,1.368)$. The differences in the rate of increase by race/ethnicity over time were not significant and as a result the interaction terms were removed from the model.

Objective 1b. Describe prostate cancer prevalence trends over time by Black subgroups (USborn, foreign born- Caribbean islanders)

When stratified analysis (by birthplace) over time (survey year) was attempted, the sample sizes per year were too small among black subgroups to report reliable annual estimates. As a result the trends observed in 1a for non-Hispanic Black men will remain the same among US-born Black men. 
Objective 1c. Document the role of ethnicity in the risk factor modeling of prostate cancer prevalence over time.

When stratified analysis over time (survey year) was attempted, the sample sizes per year were too small among black subgroups (stratified by birthplace) to perform risk factor modeling and to produce reliable results. 
Prostate cancer risk factors and PSA screening behaviors

\begin{tabular}{|c|c|c|}
\hline Characteristic & $\begin{array}{c}\text { United States } \\
\text { Weighted } n=1072667 \\
\text { Unweighted } n=192\end{array}$ & $\begin{array}{c}\text { Caribbean } \\
\text { Weighted } n=123242 \\
\text { Unweighted } n=16\end{array}$ \\
\hline \multicolumn{3}{|l|}{ Age group (\%) } \\
\hline$<45$ years & 4.79 & 2.83 \\
\hline $45+$ years & 47.06 & 69.37 \\
\hline \multicolumn{3}{|l|}{ Education(\%) } \\
\hline Some high school or less & 26.80 & 30.85 \\
\hline High school graduate & 20.72 & 33.52 \\
\hline \multicolumn{3}{|l|}{ Insurance(\%) } \\
\hline None, self pay & 5.78 & 13.28 \\
\hline Public or private & 28.68 & 47.21 \\
\hline \multicolumn{3}{|l|}{ Ever smoker(\%) } \\
\hline No & 15.54 & 23.87 \\
\hline Yes & 31.06 & 63.72 \\
\hline \multicolumn{3}{|l|}{ Physical activity(\%) } \\
\hline No & 22.73 & 42.99 \\
\hline Yes & 21.46 & 22.52 \\
\hline $\begin{array}{l}* * \text { Proportions may not equa } \\
* * * \text { Percentages are weighte }\end{array}$ & ue to missing data & \\
\hline
\end{tabular}

Among Caribbean born men aged 45+, approximately $70 \%$ reported having a PSA examination in their lifetime while among US born men aged $45+$ approximately $47 \%$ reporting ever having a PSA examination. Among US born men who were insured, approximately $29 \%$ indicated ever having a PSA exam while approximately $47 \%$ of Caribbean born insured men ever had a PSA examination. Among men who reported ever smoking, 31\% of US born men and approximately $64 \%$ of Caribbean born men had a PSA exam. 
Objective 2a: Document the association between socioeconomic status (SES) indicators and prostate cancer screening behavior

\section{Structural Equation Modeling: Socioeconomic status}

As shown in Figure 12, structural equation modeling was used for predicting the probability of PSA screening as a function of the latent variable socioeconomic status. The measurement model (confirmatory analysis performed prior to the SEM modeling) confirmed income and insurance coverage as indicator variables for the latent variable socioeconomic status. Education, in the original model as another indicator of SES, was not retained in the model. Education was highly correlated with income and its inclusion as an indicator resulted in an unstable CFA model (results not shown, both insurance and income were statistically significant $(\mathrm{p}<0.0001)$, with insurance being positively correlated with the SES latent variable and income being negatively correlated with the SES latent variable).

Figure 12. The probability of PSA screening as a function of the socioeconomic status latent variable

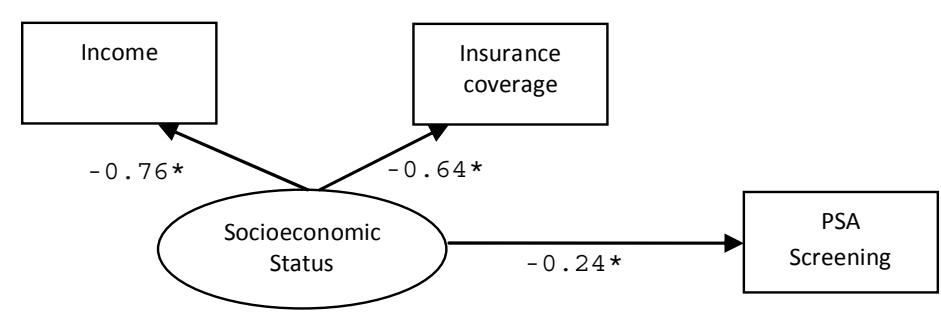

*Statistically significant path coefficient 
Table 9. Structural equation model for predicting the probability of PSA screening as a function of the socioeconomic status latent variable.

\begin{tabular}{lrrrr}
\hline Indicator Variables & Estimate & S.E. & t value & $\begin{array}{r}\text { Two Tailed } \\
\text { p-value }\end{array}$ \\
\hline Insurance & & & & 0.000 \\
Income & 0.643 & 0.045 & 14.233 & 0.000 \\
PSA - SES & -0.762 & 0.218 & -3.494 & 0.012
\end{tabular}

Measurement Models: Evaluation of goodness of fit (Coefficients)

Akaike (AIC) 5884.027

The latent variable socioeconomic status is defined by variables income and insurance. A higher score on SES indicates that the respondent was insured and had a higher income. A lower SES score indicates the respondent is uninsured and has low income. Results in Table 9 show that SES is negatively associated with PSA screening behavior. That is, the odds of PSA screening decrease as the SES latent score increases. The direct path of latent variable socioeconomic status on PSA screening is statistically significant $(\mathrm{p}<.05)$.

This model confirmed the relationship between latent variable socioeconomic status and its indicator variables as well as PSA screening behavior. Income and insurance are related factors and are commonly included in the literature as factors associated with socioeconomic status. 
Objective 2b: Explore the relationship between prostate cancer screening behavior and socioeconomic status while adjusting for age group, family history of cancer, birthplace, (USborn and Caribbean-born), and years lived in the United States.

\section{Complete mediation of the effects of exogenous variables}

As shown in Figure 13 below, a structural equation model was used to assess the effect of SES on prostate cancer screening behavior while adjusting for exogenous variables family history of cancer (yes/no), age group ( $<45$ years versus $45+$ years), birthplace (US-born versus Caribbean-born), and years lived in the United States (among those that are foreign born). The initial assumption was that the effects of the exogenous variables were completely mediated by the SES latent variable. That is, all pathways of exogenous variables (indirect effects) went through the latent variable socioeconomic status and the pathway (direct effect) of the SES latent variable went to PSA screening.

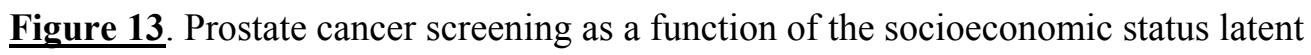
variable adjusting for the exogenous variables: Complete mediation

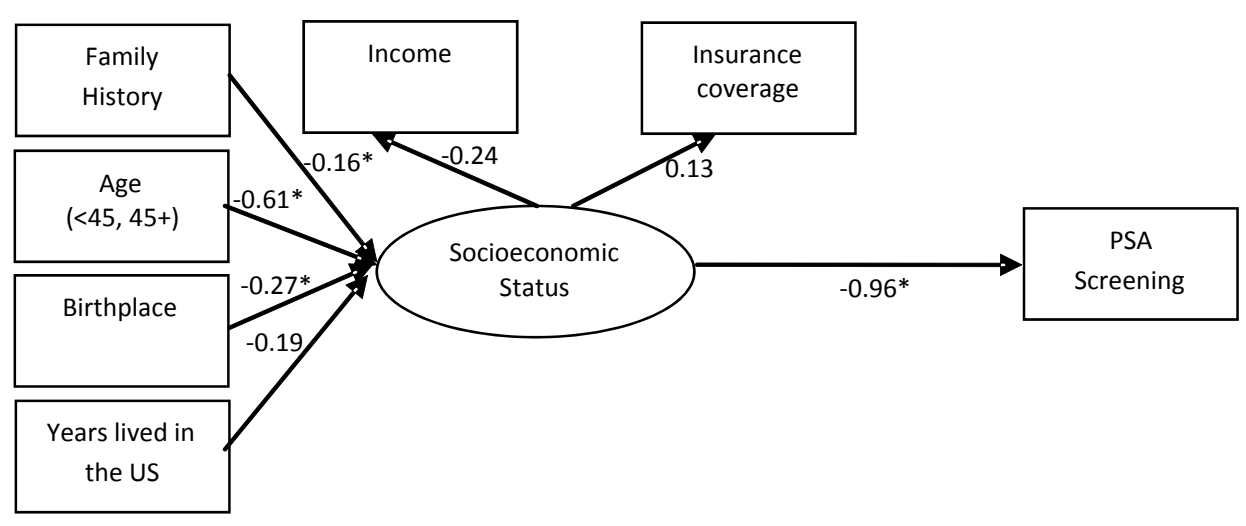

*Statistically significant path coefficient 
Table 10. Structural equation model assessing the effect of SES on prostate cancer screening behavior while adjusting for birthplace, age group, family history of cancer, and years lived in the US: Complete mediation.

\begin{tabular}{lrrrr}
\hline Indicator Variables & Estimate & S.E. & t value & $\begin{array}{r}\text { Two Tailed } \\
\text { p-value }\end{array}$ \\
\hline SES - Insurance & 0.128 & 0.087 & 1.483 & 0.138 \\
SES - Income & -0.242 & 0.141 & -1.718 & 0.086 \\
SES - Family history & -0.164 & 0.064 & -2.573 & 0.010 \\
SES - Age $(<45,45+)$ & -0.613 & 0.228 & -2.694 & 0.007 \\
SES - Birthplace & -0.265 & 0.137 & -1.929 & 0.054 \\
SES - Years lived in US & -0.186 & 0.121 & -1.539 & 0.124 \\
PSA - SES & -0.960 & 0.031 & -31.219 & 0.000
\end{tabular}

Measurement Models: Evaluation of goodness of fit (Coefficients) Akaike (AIC) 5354.772

This model intended to establish the relationship between exogenous variables family history of cancer, age group, birthplace, years lived in the US and latent variable socioeconomic status on PSA screening behavior. As in the literature the structural equation model in this study found that socioeconomic status, defined here as the combination of income and insurance coverage, was a statistically significant predictor of receipt of PSA test $(p<0.05)$. The addition of exogenous factors family history of cancer, age group, birthplace, and years lived in the US, completely mediated by latent variable socioeconomic status increased the odds of a man not receiving a PSA test (odds ratio increased from 1.344 to 2.119). The model fit changed indicating a better fit $(\mathrm{AIC}=5354.772$ compared to 5884.027). This model also showed that all exogenous variables were negatively associated with the latent SES variable. They were all statistically significant (or for birthplace very close to the significance level) except for the number of years lived in the US. 
Figure 14. Prostate cancer screening as a function of the socioeconomic status latent variable, adjusting for exogenous variables, except for the number of years lived in the US: Complete mediation

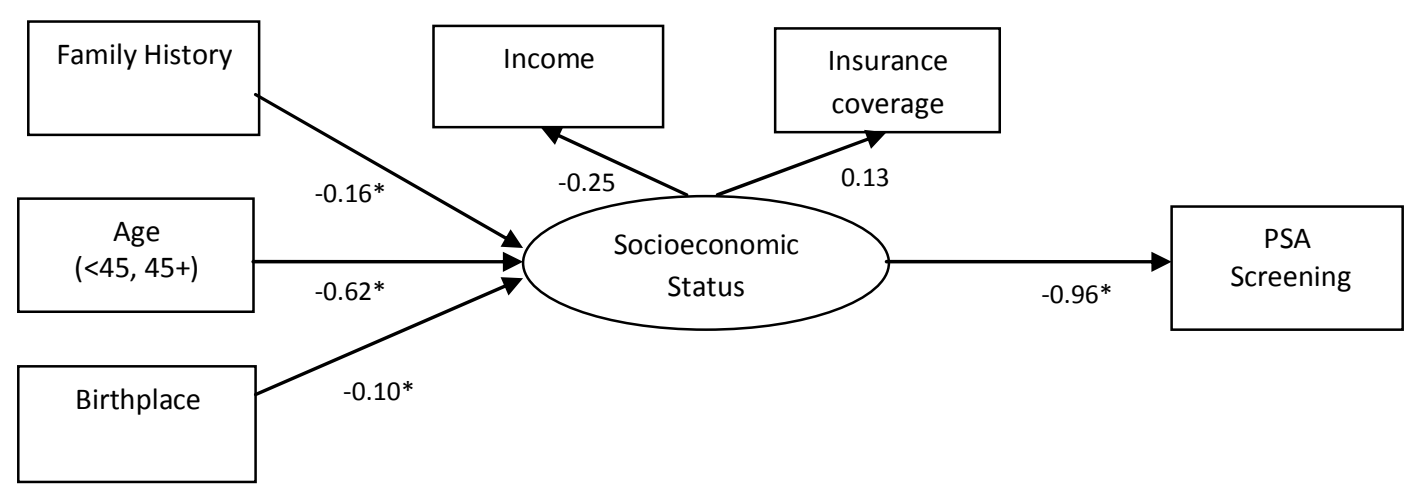

*Statistically significant path coefficient

Table 11. Structural equation model assessing the effect of SES on prostate cancer screening behavior while adjusting for birthplace, age group, and family history of cancer: Complete mediation.

\begin{tabular}{lrrrr}
\hline Indicator Variables & Estimate & S.E. & t value & $\begin{array}{r}\text { Two Tailed } \\
\text { p-value }\end{array}$ \\
\hline SES - Insurance & 0.133 & 0.091 & 1.469 & 0.142 \\
SES - Income & -0.249 & 0.141 & -1.772 & 0.076 \\
SES - Family history & -0.163 & 0.063 & -2.605 & 0.009 \\
SES - Age $(<45,45+)$ & -0.616 & 0.225 & -2.742 & 0.006 \\
SES - Birthplace & -0.100 & 0.039 & -2.542 & 0.011 \\
PSA - SES & -0.962 & 0.028 & -34.822 & 0.000
\end{tabular}

Measurement Models: Evaluation of goodness of fit (Coefficients) Akaike (AIC) 5357.297

In an attempt to improve model fit from Figure 13, years lived in the US was removed from the model in Figure 14. This model assessed the relationship between exogenous variables 
family history, age, and birthplace and PSA screening completely mediated by SES. The AIC value increased slightly from 5354 in Figure 13 to 5357 in Figure 14 which indicates that the removal of years lived in the US did not have a large impact in improving model fit. In this model, the significant inverse relationship between SES and PSA screening remained the same. Family history of cancer, age group, and birthplace all had significant inverse relationships with latent variable socioeconomic status as in the previous model (Figure 13). The removal of years lived in the US changed the partial effect of birthplace on latent variable SES, which decreased in absolute value, but was statistically significant.

\section{Partial mediation of the effects of exogenous variables}

Both indirect effects (mediated by latent variable SES) and direct effects of exogenous variables on to PSA screening were allowed in this model, along with the direct effect of the SES latent variable (see pathways in Figure 15). 
Figure 15. Prostate cancer screening as a function of the socioeconomic status latent variable adjusting for the exogenous variables: Partial mediation

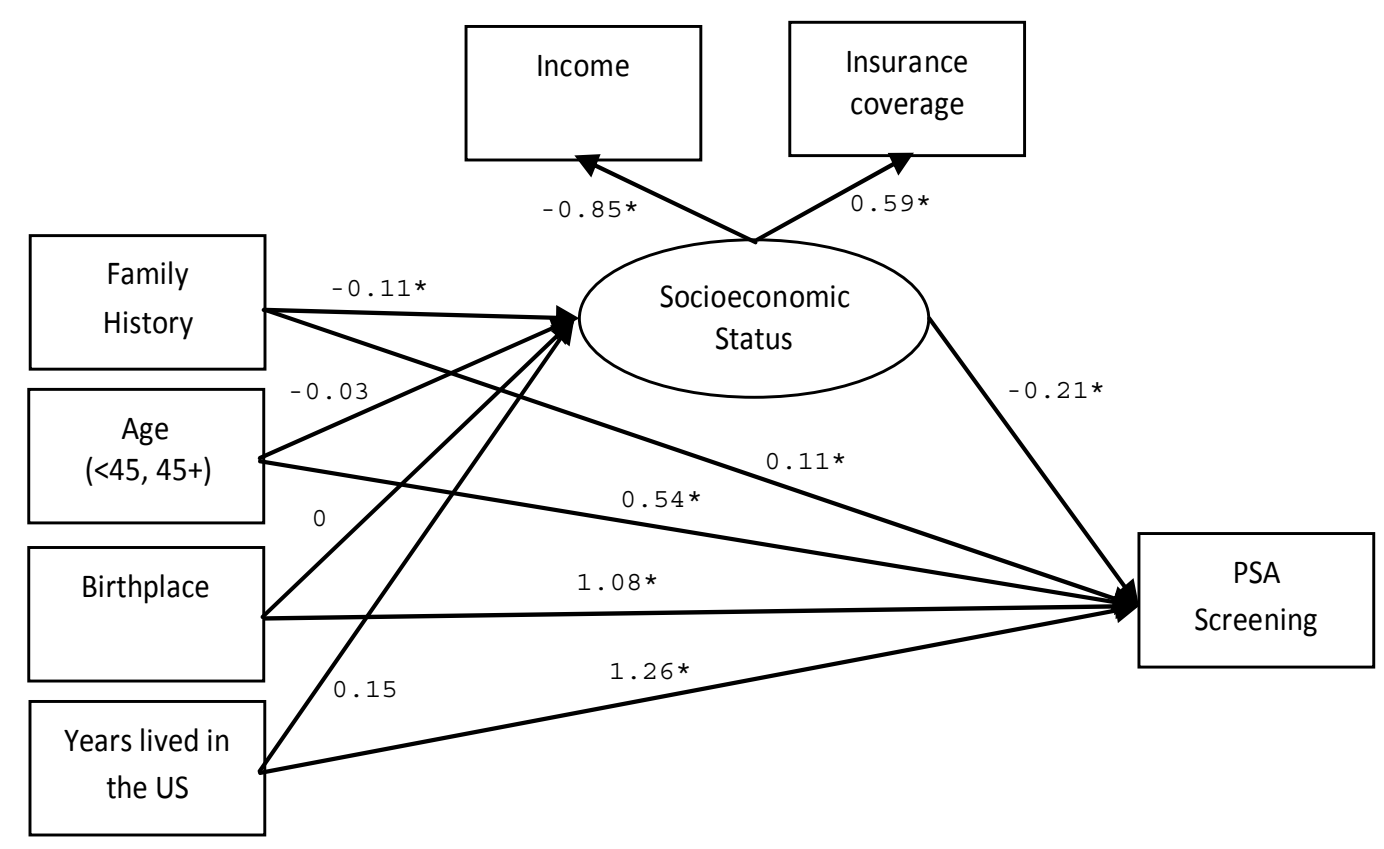

*Statistically significant path coefficient

Table 12. Structural equation model for assessing the effect of SES on prostate cancer screening behavior while adjusting for birthplace, age group, family history of cancer, and years lived in the US: Partial mediation

\begin{tabular}{lrrrr}
\hline Indicator Variables & Estimate & S.E. & t value & $\begin{array}{r}\text { Two Tailed } \\
\text { p-value }\end{array}$ \\
\hline SES - Insurance & 0.592 & 0.055 & 10.786 & 0.000 \\
SES - Income & -0.853 & 0.236 & -3.616 & 0.000 \\
SES - Family history & -0.111 & 0.049 & -2.272 & 0.023 \\
SES - Age $(<45,45+)$ & -0.033 & 0.049 & -0.676 & 0.499 \\
SES - Birthplace & 0.001 & 0.225 & 0.004 & 0.997 \\
SES - Years lived in US & 0.155 & 0.205 & 0.757 & 0.449 \\
PSA - SES & -0.207 & 0.068 & -3.035 & 0.002 \\
PSA - Family history & 0.109 & 0.027 & 3.970 & 0.000 \\
\hline
\end{tabular}




\begin{tabular}{lcccr}
\hline Indicator Variables & Estimate & S.E. & t value & $\begin{array}{r}\text { Two Tailed } \\
\text { p-value }\end{array}$ \\
& & & & 0.000 \\
PSA - Age group & 0.539 & 0.030 & 18.162 & 0.000 \\
PSA - Birthplace & $1.078 \dagger$ & 0.057 & 18.942 & 0.000 \\
PSA - Years lived in US & $1.255 \dagger$ & 0.064 & 19.648 & \\
& & & \\
Measurement Models: Evaluation of goodness of fit (Coefficients) & \\
Akaike (AIC) & 5230.676 \\
$\dagger$ Due to problems with convergence this value could not be accurately computed & \\
\hline
\end{tabular}

In this model (Figure 15) where each of the exogenous variables had a direct path to PSA, the AIC value decreased from 5354.772 (figure 13) to 5230.676 indicating a better fit [72]. Although this model was a better fit, the path between age group and SES $(\mathrm{p}=0.499, \mathrm{t}=-0.676)$, birthplace and SES $(\mathrm{p}=0.997, \mathrm{t}=-0.004)$, and years lived in the US and SES $(\mathrm{p}=0.449, \mathrm{t}=-0.757)$, were not significant in the model. The direct paths from each exogenous variable (family history, age group, birthplace, and years lived in the US) to PSA screening were all positive and statistically significant $(\mathrm{p}<.05)$. In this model, for the variables birthplace and years lived in the US, the direct path coefficients to PSA did not converge properly, leading to standardized coefficient values greater than 1 . As a result these paths did not produce interpretable standardized estimates. 
Figure 16. Prostate cancer screening as a function of the socioeconomic status latent variable, adjusting for the exogenous variables, except for the number of years lived in the US:

Partial mediation

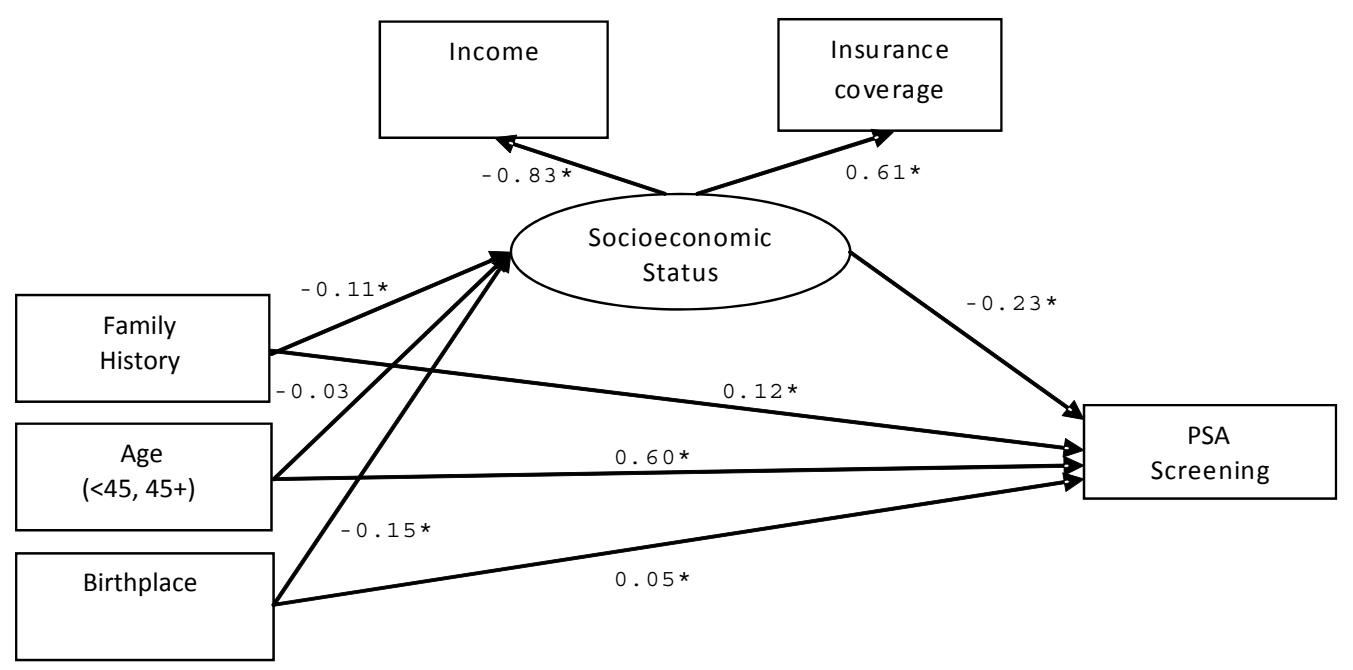

*Statistically significant path coefficient

Table 13. Structural equation model for assessing the effect of SES on prostate cancer screening behavior while adjusting for birthplace, age group, family history of cancer, and years lived in the US: Partial mediation

\begin{tabular}{lrrrr}
\hline Indicator Variables & Estimate & S.E. & t value & $\begin{array}{r}\text { Two Tailed } \\
\text { p-value }\end{array}$ \\
\hline SES - Insurance & 0.611 & 0.039 & 15.840 & 0.000 \\
SES - Income & -0.825 & 0.157 & -5.255 & 0.000 \\
SES - Family history & -0.110 & 0.047 & -2.351 & 0.019 \\
SES - Age (<45, 45+) & -0.031 & 0.049 & -0.626 & 0.532 \\
SES - Birthplace & -0.145 & 0.053 & -2.721 & 0.007 \\
PSA - SES & -0.227 & 0.069 & -3.288 & 0.001 \\
PSA - Family history & 0.118 & 0.030 & 3.934 & 0.000 \\
PSA - Age group & 0.596 & 0.029 & 20.263 & 0.000 \\
PSA - Birthplace & 0.053 & 0.023 & 2.298 & 0.022 \\
& & & \\
Measurement Models: Evaluation of goodness of fit (Coefficients) & & \\
Akaike (AIC) & 5234.029 & & \\
\hline
\end{tabular}


In an effort to improve the model, the variable years lived in the US was excluded. In this new model, (Figure 16), the paths from exogenous variables family history and birthplace to socioeconomic status are significant and negative indicating an inverse relationship with SES. The path from age to socioeconomic status is also negative but not significant. Of the paths to PSA, SES had an inverse significant relationship, as observed earlier. All other paths from family history, age group, and birthplace to PSA are significant and have a positive relationship. The AIC changed from 5230 to 5234 which is a small change. Figure 15 is a slightly better fitting model since it has a lower AIC of 5230. However, since some of the loadings did not converge properly in Figure 15, Figure 16 results indicate this is a better model.

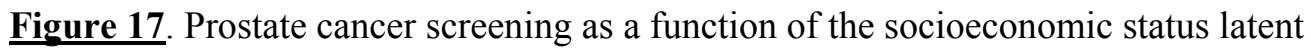
variable adjusting for the exogenous variables (family history of cancer, birthplace, years lived in the US: Partial mediation

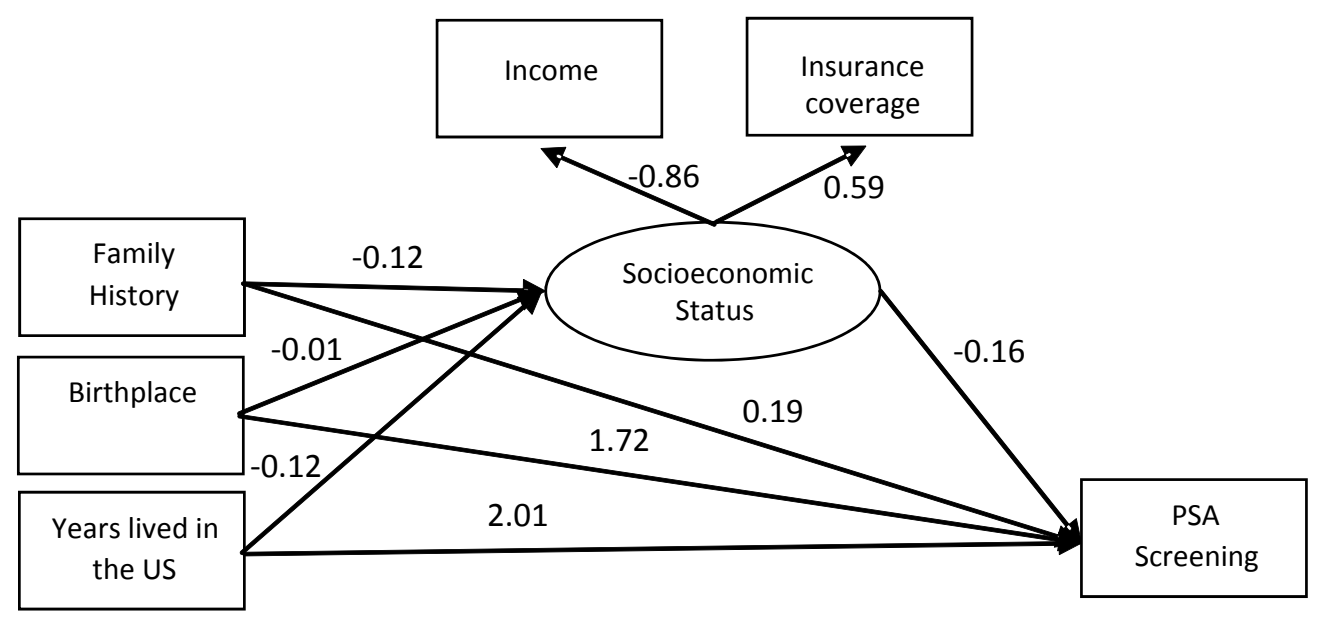

*Statistically significant path coefficient 
Table 14. Structural equation model of exogenous variables (family history of cancer, birthplace, and years lived in the United States), latent variable socioeconomic status, and PSA screening: Partial mediation

\begin{tabular}{|c|c|c|c|c|}
\hline Indicator Variables & Estimate & S.E. & t value & $\begin{array}{l}\text { Two Tailed } \\
\text { p-value }\end{array}$ \\
\hline SES - Income & -0.857 & 0.127 & -6.739 & 0.000 \\
\hline SES - Insurance & 0.587 & 0.036 & 16.494 & 0.000 \\
\hline SES - Family history & -0.119 & 0.046 & -2.581 & 0.010 \\
\hline SES - Birthplace & -0.007 & 0.154 & -0.047 & 0.962 \\
\hline SES - Years lived in US & 0.149 & 0.139 & 1.071 & 0.284 \\
\hline PSA - SES & -0.156 & 0.051 & -3.091 & 0.002 \\
\hline PSA - Family history & 0.187 & 0.024 & 7.663 & 0.000 \\
\hline PSA - Birthplace & $1.721 \dagger$ & 0.049 & 35.482 & 0.000 \\
\hline PSA - Years lived in US & $2.007 \dagger$ & 0.043 & 46.178 & 0.000 \\
\hline
\end{tabular}

Measurement Models: Evaluation of goodness of fit (Coefficients)

Akaike (AIC) 5631.415

$\dagger$ Due to problems with convergence this value could not be accurately computed

In an effort to improve model fit, age group (a non significant contributor to the model in Figure 15), was removed from the model. The majority of men who received a PSA exam were older than age 45 which would support little variation by age group. Removing age group however did not improve model fit (AIC:5631 versus AIC:5230). Since removing this variable from the model did not improve model fit it was later put back into the model.

\section{PSA screening and Socioeconomic Status within Caribbean-born}

\section{Complete mediation of the effects of exogenous variables}

In this section (Figure 18) we explored the relationship between prostate cancer screening behavior and socioeconomic status for the Caribbean-born group only while adjusting for age group, family history of cancer, and Caribbean birthplace. The effects of exogenous variables were mediated by SES latent variable, and the direct effect of SES latent variable on PSA screening was estimated. 
Figure 18. The prostate cancer screening for Caribbean-born group as a function of the socioeconomic status latent variable adjusting for the exogenous variables: Complete mediation

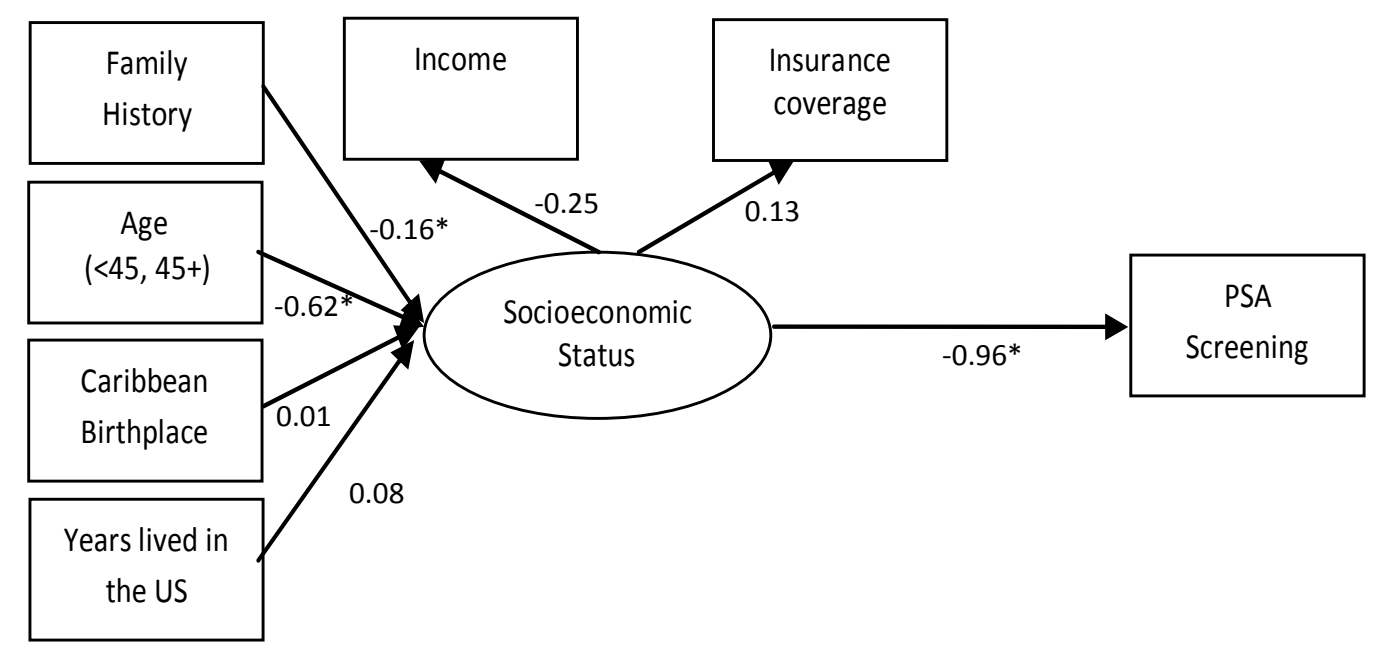

*Statistically significant path coefficient

Table 15. Structural equation model assessing the effect of SES on prostate cancer screening behavior for the Caribbean-born group while adjusting for family history of cancer, age group, Caribbean birthplace, and years lived in the US: Complete mediation

\begin{tabular}{lrrrr}
\hline Indicator Variables & Estimate & S.E. & t value & $\begin{array}{r}\text { Two Tailed } \\
\text { p-value }\end{array}$ \\
\hline SES - Income & -0.249 & 0.140 & -1.777 & 0.076 \\
SES - Insurance & 0.132 & 0.089 & 1.479 & 0.139 \\
SES - Family history & -0.163 & 0.062 & -2.605 & 0.009 \\
SES - Age $(<45,45+)$ & -0.619 & 0.226 & -2.739 & 0.006 \\
SES - Caribbean birthplace & 0.006 & 0.075 & 0.073 & 0.942 \\
SES - Years lived in the US & 0.076 & 0.066 & 1.151 & 0.250 \\
PSA - SES & -0.962 & 0.028 & -34.620 & 0.000
\end{tabular}

Measurement Models: Evaluation of goodness of fit (Coefficients)

Akaike (AIC) $\quad 5360.797$ 
This model (Figure 18) confirmed the relationship between exogenous variables (family history of cancer, age group, Caribbean birthplace, and years lived in the United States) and latent variable SES on PSA screening behavior. Interestingly, neither Caribbean birthplace nor years lived in the United States had a significant effect on socioeconomic status.

The model results are similar to those obtained previously for all men, except that birthplace and years lived in the US were negatively associated with the SES latent variable, contrary to the observed positive associations for Caribbean birthplace and years lived in the US in the present model. The latent variable SES was negatively associated with PSA as observed earlier (Figure $13 \&$ Table 10), and the magnitude of the effect is similar (no difference in effects of SES on PSA between all men group and the Caribbean-born group).

\section{Structural equation model for the Caribbean-born group: Partial mediation of the effects of exogenous variables}

Figure 19 illustrates both indirect effects (mediated by latent variable SES) and direct effects of exogenous variables on to PSA screening for the Caribbean-born group, along with the direct effect of the SES latent variable onto PSA screening. 
Figure 19. The prostate cancer screening as a function of the socioeconomic status latent variable for Caribbean-born group adjusting for the exogenous variables: Partial mediation

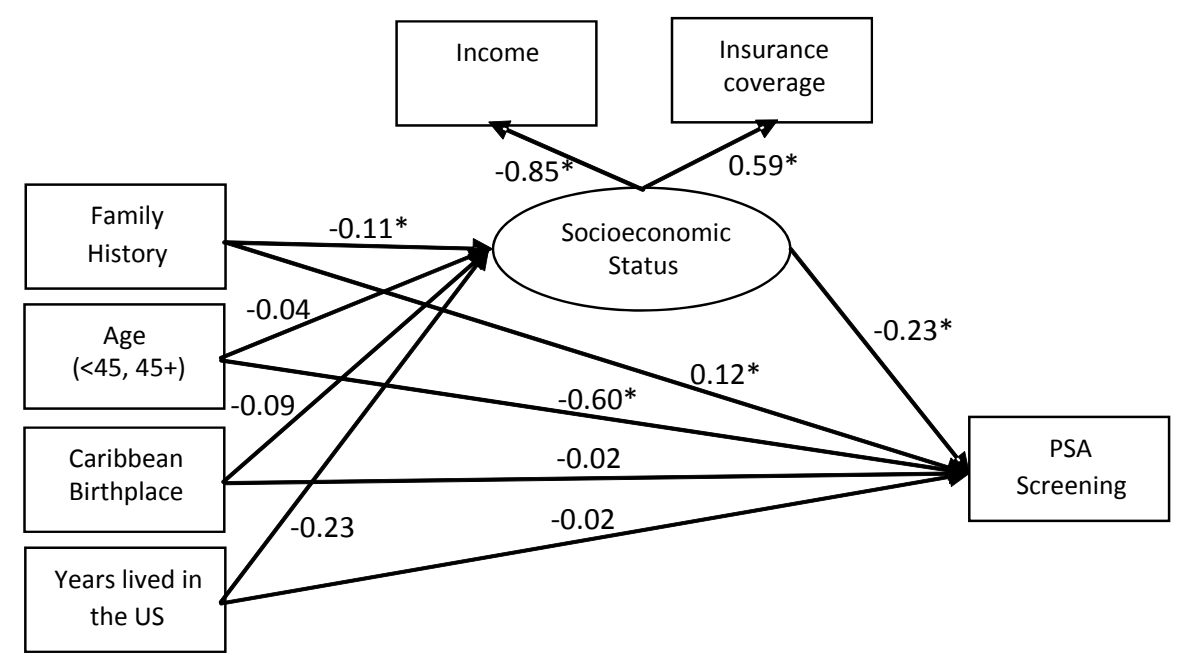

*Statistically significant path coefficient

Table 16. Structural equation model assessing the effect of SES on prostate cancer screening behavior for the Caribbean-born group while adjusting for family history of cancer, age group, Caribbean birthplace, and years lived in the US: Partial mediation

\begin{tabular}{lrrrr}
\hline Indicator Variables & Estimate & S.E. & t value & $\begin{array}{r}\text { Two Tailed } \\
\text { p-value }\end{array}$ \\
\hline SES - Insurance & & & & 0.000 \\
SES - Income & 0.594 & 0.047 & 12.616 & 0.000 \\
SES - Family history & -0.852 & 0.191 & -4.452 & 0.021 \\
SES - Age $(<45,45+)$ & -0.109 & 0.048 & -2.299 & 0.470 \\
SES - Caribbean birthplace & -0.036 & 0.049 & -0.723 & 0.533 \\
SES - Years lived in US & -0.086 & 0.139 & -0.623 & 0.062 \\
PSA - SES & 0.229 & 0.123 & 1.865 & 0.001 \\
PSA - Family history & -0.229 & 0.069 & -3.305 & 0.000 \\
PSA - Age (<45, 45+) & 0.118 & 0.030 & 3.886 & 0.000 \\
PSA - Caribbean birthplace & 0.598 & 0.030 & 20.089 & 0.573 \\
PSA - Years lived in US & -0.020 & 0.036 & -0.564 & 0.665
\end{tabular}

Measurement Models: Evaluation of goodness of fit (Coefficients) Akaike (AIC) 5237.468 
As observed previously, partial mediation improved the model as this model showed better model fit than the last (AIC: 5237 compared to 5360). The odds of being screened for prostate cancer among Caribbean men with a family history of cancer was two times greater which is similar to that seen in Figure 13 which included US-born men. Among the Caribbeanborn men, age group did not have a significant relationship with socioeconomic status but did however have a significant impact on PSA screening directly. Caribbean birthplace did not have a significant relationship with socioeconomic status nor with PSA screening directly.

In summary, between all the models relating SES to PSA while adjusting for the different exogenous variables, Figure 16 (and Table 13) and Figure 19 (Table 16) present the models that would be recommended (the best choices) for non-Hispanic Black men and for Caribbean-born men respectively. This recommendation is based on both the AIC criteria as well as the fact that the convergence was perfectly reached.

Objective 2c: Document the association between lifestyle indicators and prostate cancer screening behavior

\section{Structural Equation Modeling: Lifestyle}

A confirmatory factor analysis was performed to confirm the measurement model for the latent variable lifestyle prior to exploring the relationship between lifestyle and screening behavior. The lifestyle latent variable estimates as defined by smoking status, physical activity, and BMI as indicators did not terminate normally. The measurement model resulted in a nonpositive definite fisher information matrix (leading to standard errors not being computed), and as a result BMI was removed from the model due to its strong correlation with both physical activity and smoking. BMI was chosen to be excluded from the model as this variable was an indirect 
measure of diet which is a known factor in the risk of developing cancer. The other two indicator variables smoking status and physical activity are named risk factors in the development of cancer and these two variables were directly measured in the survey, unlike BMI which was a planned proxy for diet.

As a result, the model which left indicator variables smoking status and physical activity to define latent variable lifestyle led to a better fitting model which terminated normally. Both smoking status and physical activity were statistically significant $(\mathrm{p}<0.001)$, with smoking status being positively correlated with the latent SES variable and physical activity being negatively correlated with the latent SES variable (results not shown).

Figure 20. Structural equation model for predicting the probability of PSA screening as a function of the latent variable lifestyle

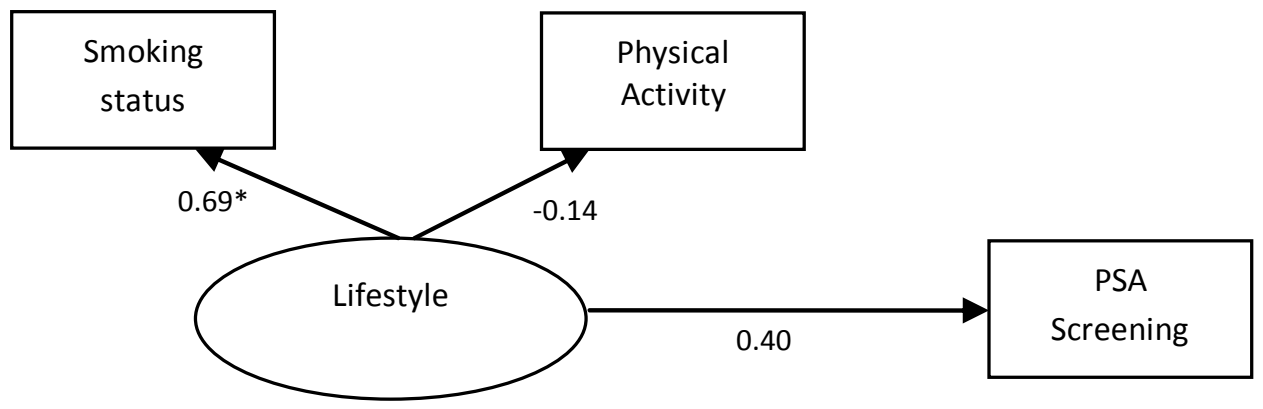

*Statistically significant path coefficient 
Table 17. Structural equation model for predicting the probability of PSA screening as a function of the latent variable lifestyle.

\begin{tabular}{|c|c|c|c|c|}
\hline Indicator Variables & Estimate & S.E. & $\mathrm{t}$ value & $\begin{array}{r}\text { Two Tailed } \\
\text { p-value }\end{array}$ \\
\hline LS - Smoking status & 0.691 & 0.060 & 11.469 & 0.000 \\
\hline LS - Physical activity & -0.135 & 0.082 & -1.641 & 0.101 \\
\hline PSA - Lifestyle & 0.401 & 0.250 & 1.602 & 0.109 \\
\hline \multicolumn{5}{|c|}{ Measurement Models: Evaluation of goodness of fit (Coefficients) } \\
\hline
\end{tabular}

As shown in Figure 20, the direct effect of the latent lifestyle variable on PSA screening was assessed. Lifestyle factors such as smoking and physical activity are well established as factors that impact the risk of cancer. Therefore one would assume that if you smoke and are not physically active you would be at higher risk for developing prostate cancer and should therefore be monitored more closely thereby more likely to be screened. Often times the opposite is true however, you find that people who engage in high-risk activity are generally less likely to have optimal health care seeking behaviors.

Results in Table 17 show that the latent variable lifestyle is not a statistically significant predictor of prostate cancer screening, although the odds of PSA screening increases with the increase in healthy lifestyle scores $(\mathrm{OR}=1.582)$.

Objective 2d: Explore the relationship between prostate cancer screening behavior and lifestyle indicators while adjusting for age group, family history of cancer, birthplace (US born and Caribbean-born), and years lived in the United States (among those foreign born) 


\section{Complete mediation of the effects of exogenous variables}

The first model examined was intended to assess the relationship between latent variable lifestyle, defined by indicator variables smoking status and physical activity, while adjusting for exogenous variables family history of cancer, age group $(<45,45+)$, birthplace, and years lived in the US and assuming complete mediation of the effects of exogenous variables on PSA screening. This model however, did not yield any results due to convergence problems. A decision was made to drop one of the exogenous variables, years lived in the US, and rerun the model (Figure 21).

Results (Table 18) clearly show an improvement in the model fit when comparing this model to the previous model with only the lifestyle latent variable (AIC: 5117.299 compared to 5805.011). Results also show a strong positive and statistically significant effect of lifestyle on PSA screening likelihood, with the odds of screening increasing with the lifestyle scores $(\mathrm{OR}=28.854)$. The removal of years lived in the United States provided results with variables family history of cancer, and age group associated significantly to lifestyle, but with the variable birthplace at the borderline of significance $(\mathrm{p}=0.07)$. 
Figure 21. Prostate cancer screening as a function of the latent variable lifestyle, adjusting for exogenous variables, except the number of years lived in the US: Complete mediation

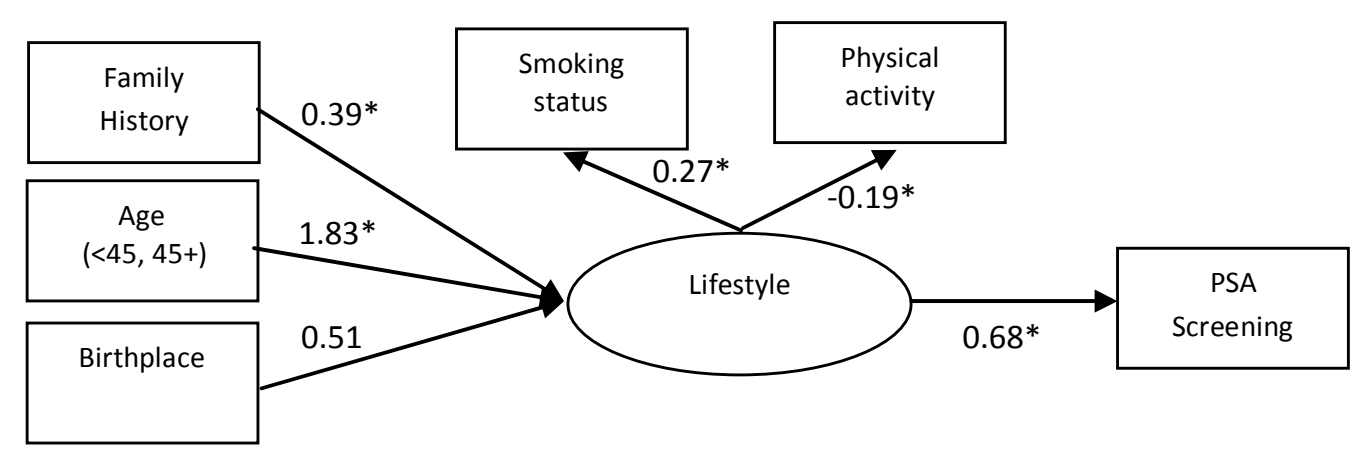

*Statistically significant path coefficient

Table 18. Structural equation model assessing the effect of the lifestyle latent variable on the prostate cancer screening while adjusting for family history of cancer, age group, and birthplace: Complete mediation

\begin{tabular}{lrrrr}
\hline Indicator Variables & Estimate & S.E. & t value & $\begin{array}{r}\text { Two Tailed } \\
\text { p-value }\end{array}$ \\
\hline LS - Smoking status & 0.267 & 0.101 & 2.641 & 0.008 \\
LS - Physical activity & -0.190 & 0.057 & -3.354 & 0.001 \\
LS - Family history & 0.394 & 0.159 & 2.477 & 0.013 \\
LS - Age (<45, 45+) & $1.829 \dagger$ & 0.618 & 2.960 & 0.003 \\
LS - Birthplace & 0.507 & 0.281 & 1.804 & 0.071 \\
PSA - LS & 0.681 & 0.150 & 4.540 & 0.000 \\
Measurement Models: Evaluation of goodness of fit (Coefficients) & & \\
Akaike (AIC) & 5117.299 \\
$\dagger$ & & \\
\end{tabular}




\section{Partial mediation of the effects of exogenous variables}

Pathways from exogenous variables to both latent variable lifestyle (indirect effects: latent variable lifestyle being the mediator) and PSA screening (direct effects) were allowed in the model, along with the pathway from the latent variable lifestyle to PSA screening (see Figure 22). As for the previous model, years lived in the US was removed from the exogenous variables as it caused model misspecification (leading to non convergence of the model estimation).

Figure 22. Prostate cancer screening as a function of the latent variable lifestyle, while adjusting for exogenous variables, except the number of years lived in the US: Partial mediation

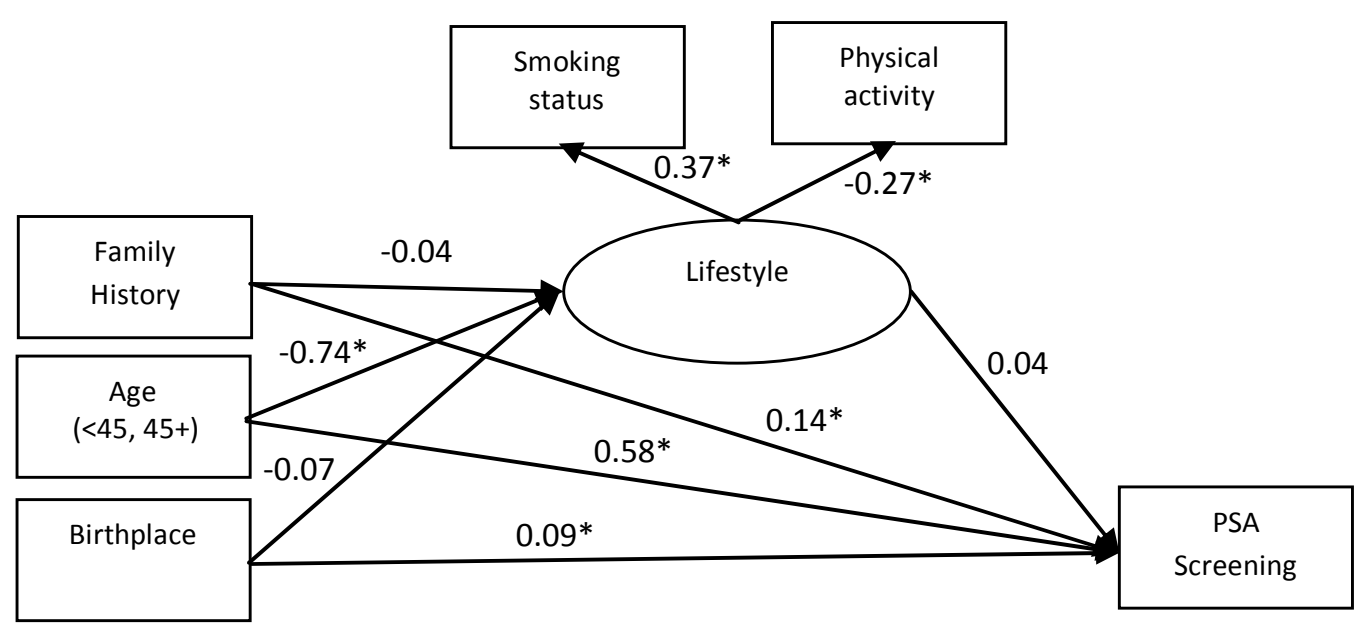

*Statistically significant path coefficient 
Table 19. Structural equation model assessing the effect of the lifestyle latent variable on the prostate cancer screening while adjusting for family history of cancer, age group, and birthplace:

Partial mediation

\begin{tabular}{lrrrr}
\hline Indicator Variables & Estimate & S.E. & t value & $\begin{array}{r}\text { Two Tailed } \\
\text { p-value }\end{array}$ \\
\hline LS - Smoking status & & & & 0.000 \\
LS - Physical activity & 0.370 & 0.093 & 3.956 & 0.000 \\
LS - Family history & -0.269 & 0.075 & -3.575 & 0.561 \\
LS - Age $(<45,45+)$ & -0.043 & 0.073 & -0.582 & 0.000 \\
LS - Birthplace & 0.737 & 0.167 & 4.404 & 0.343 \\
PSA - LS & -0.071 & 0.075 & -0.948 & 0.874 \\
PSA - Family history & 0.036 & 0.227 & 0.159 & 0.000 \\
PSA - Age $(<45,45+)$ & 0.139 & 0.031 & 4.521 & 0.001 \\
PSA - Birthplace & 0.576 & 0.171 & 3.373 & 0.003
\end{tabular}

Measurement Models: Evaluation of goodness of fit (Coefficients)

Akaike (AIC) $\quad 5109.526$

The addition of direct effects of the exogenous variables onto PSA screening produced better results indicating that in this sample the identified risk factors such as family history of cancer, age group, and birthplace were better predictors of prostate cancer screening activity than the latent variable lifestyle. This was first evidenced by the change in the AIC value from 5117.299 for the model where the exogenous variables were completely mediated by lifestyle to 5109.526 in this model with direct effects (Table 19). Results show strong positive and statistically significant effects of the exogenous variables on PSA screening, with corresponding odds ratio values of $2.236,16.432$, and 3.304 for family history of cancer, age group, and birthplace respectively.

Interestingly, family history of cancer no longer exhibited a significant effect on the latent variable lifestyle, nor did birthplace. As expected the variable "age group" remained significantly associated with lifestyle. Notice that the effect of the latent variable lifestyle on PSA 
screening changed drastically with the inclusion of direct effects to PSA screening, with corresponding odds ratio dropping from $\mathrm{OR}=28.854$ to 1.128 (Tables 18 and 19).

\section{PSA screening and Lifestyle within Caribbean-born}

\section{Complete mediation of the effects of exogenous variables}

As done for the larger group, the effects of exogenous variables (family history of cancer, years lived in the US and Caribbean birthplace) were mediated by the latent variable lifestyle, and the direct effect of the latent variable lifestyle on PSA screening was estimated.

Figure 23. Structural equation model for prostate cancer screening as a function of the latent variable lifestyle for the Caribbean-born group, adjusting for exogenous variables: Complete mediation

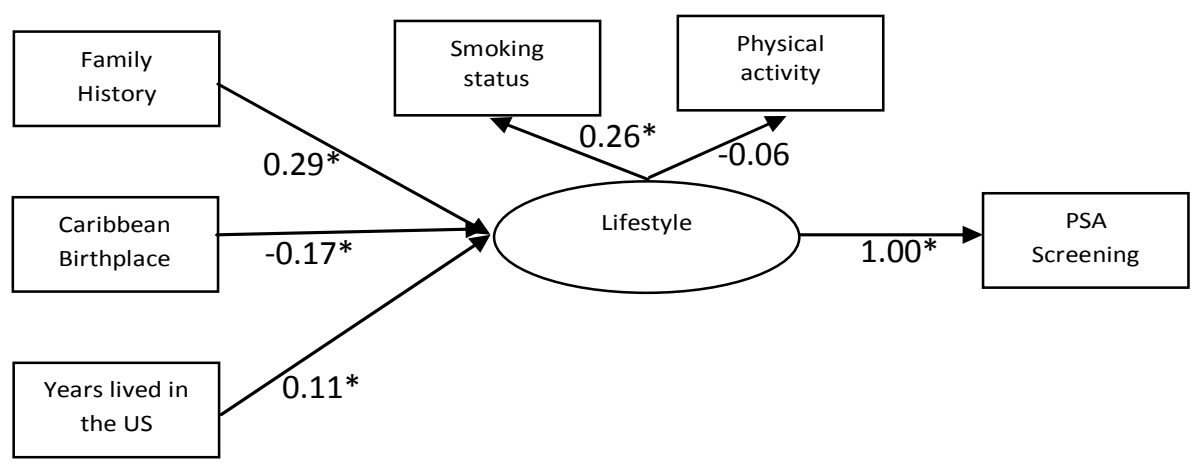

*Statistically significant path coefficient 
Table 20. Structural equation model assessing the effect of the latent variable lifestyle on prostate cancer screening while adjusting for family history of cancer, Caribbean birthplace, and years lived in the United States: Complete mediation

\begin{tabular}{|c|c|c|c|c|}
\hline Indicator Variables & Estimate & S.E. & t value & $\begin{array}{l}\text { Two Tailed } \\
\text { p-value }\end{array}$ \\
\hline LS - Smoking status & 0.263 & 0.042 & 6.183 & 0.000 \\
\hline LS - Physical activity & -0.059 & 0.049 & -1.184 & 0.236 \\
\hline LS - Family history & 0.285 & 0.006 & 48.256 & 0.000 \\
\hline LS - Caribbean birthplace & -0.174 & 0.016 & -10.829 & 0.000 \\
\hline LS - Years lived in US & 0.117 & 0.010 & 11.452 & 0.000 \\
\hline PSA - Lifestyle & $1.000 \dagger$ & 0.000 & 17482.426 & 0.000 \\
\hline \multicolumn{5}{|c|}{$\begin{array}{l}\text { Akaike (AIC) } \\
* * \text { LS = latent variable lifestyle } \\
+ \text { Due to problems with convergence this value could not be accurately computed }\end{array}$} \\
\hline
\end{tabular}

Among Caribbean-born men, the variable age group was removed from the model as an exogenous variable since within this group all men who were screened were older than age 45 . Since all the men screened were older than age 45 , this caused convergence problems in model fitting. Within the modified model, all exogenous variables (family history of cancer, Caribbean birthplace, and years lived in the US) were significantly associated with the latent variable lifestyle. Moreover, the observed effect of the latent variable lifestyle on PSA screening is stronger for the Caribbean-born men than for the larger group (all non-Hispanic Black men) (Tables $18 \& 20)$.

\section{Structural equation model for Caribbean-born group: Partial mediation of the effects of exogenous variables}

This model explored the relationship between exogenous variables family history of cancer, age group, Caribbean birthplace, and years lived in the United States with latent variable lifestyle and prostate cancer screening. In this model, indirect and direct effects of exogenous 
variables as well as direct effect of latent variable lifestyle on PSA screening were examined. All the exogenous variables were included in the model.

Figure 24. Structural equation model for prostate cancer screening as a function of the latent variable lifestyle for the Caribbean-born group, adjusting for the exogenous variables:

Partial mediation

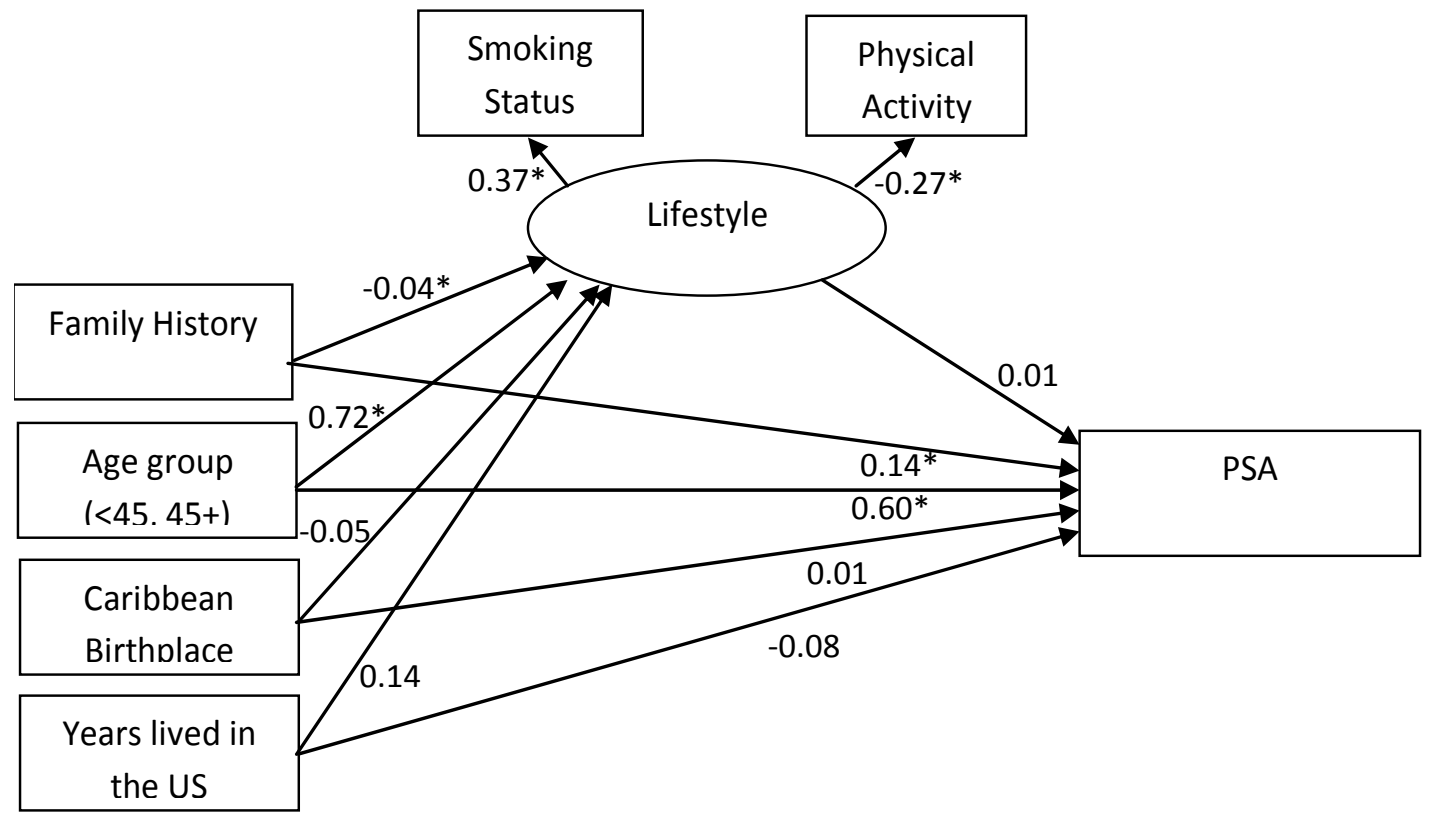

*Statistically significant path coefficient

Table 21. Structural equation model assessing the effect of the latent variable lifestyle on prostate cancer screening while adjusting for family history of cancer, age group, Caribbean birthplace, and years lived in the United States: Partial mediation

\begin{tabular}{lrrrr}
\hline Indicator Variables & Estimate & S.E. & t value & $\begin{array}{r}\text { Two Tailed } \\
\text { p-value }\end{array}$ \\
\hline LS - Smoking status & & & & 0.000 \\
LS - Physical activity & 0.374 & 0.094 & 3.969 & 0.000 \\
LS - Family history & -0.273 & 0.069 & -3.936 & 0.570 \\
\hline
\end{tabular}


Table 21. Structural equation model assessing the effect of the latent variable lifestyle on prostate cancer screening while adjusting for family history of cancer, age group, Caribbean birthplace, and years lived in the United States: Partial mediation (continued)

\begin{tabular}{lrrrr}
\hline Indicator Variables & Estimate & S.E. & t value & $\begin{array}{r}\text { Two Tailed } \\
\text { p-value }\end{array}$ \\
\hline LS - Age $(<45,45+)$ & 0.724 & 0.157 & 4.622 & 0.000 \\
LS - Caribbean birthplace & -0.045 & 0.127 & -0.350 & 0.726 \\
LS - Years lived in the US & 0.140 & 0.118 & 1.188 & 0.235 \\
PSA - Lifestyle & 0.012 & 0.223 & 0.055 & 0.956 \\
PSA - Family history & 0.137 & 0.030 & 4.510 & 0.000 \\
PSA - Age $(<45,45+)$ & 0.597 & 0.164 & 3.649 & 0.000 \\
PSA - Caribbean birthplace & 0.005 & 0.062 & 0.074 & 0.941 \\
PSA - Years lived in the US & -0.075 & 0.062 & -1.193 & 0.223
\end{tabular}

Measurement Models: Evaluation of goodness of fit (Coefficients) Akaike (AIC) 5112.891

In this model the indicator variables smoking status and physical activity produced significant results with a p-value less than 0.0001 for both variables. Among the exogenous variables with paths to the latent variable lifestyle, age group was the only variable significantly associated with the latent variable lifestyle with a positive strong correlation (p-value $<0.001$ ). Caribbean birthplace and family history of cancer showed negative but non-significant associations with lifestyle, while years lived in the United States, although non-significant, was positively associated with lifestyle. As for paths to PSA screening, only the paths from family history and age group were significant $(p$-value $<0.001)$ with strong positive effects. Family history and cancer and age $45+$ increased the odds of PSA screening $(\mathrm{OR}=2.208, \& \mathrm{OR}=$ 18.125), respectively. Although non-significant, lifestyle and Caribbean birthplace were positively associated with PSA screening, while years lived in the United States was inversely associated with PSA screening behavior. 
Objective 2e: Explore the relationship between prostate cancer screening behavior, latent variables socioeconomic status and lifestyle, exogenous variables age group, family history of cancer, birthplace, and years lived in the United States

The following models explored the effects of both latent variables SES and lifestyle while controlling for the effects of exogenous variables (see Figure 7). These full models assumed that effects of the exogenous variables were partially mediated by both SES and lifestyle (pathways from these variables to both SES and lifestyle), and also allowed direct pathways to PSA screening. We considered two options: (a) models without covariation between both latent variables, and (b) models with covariation between latent variables SES and lifestyle.

\section{Partial mediation of the effects of exogenous variables: Full Model}

The full model assesses the big picture and includes both measurement models for

lifestyle and socioeconomic status. All prostate cancer risk factors (family history of cancer, age group, birthplace, and years lived in the United States) are included. 


\section{Partial mediation of the effects of exogenous variables without covariation between latent}

variables SES and lifestyle

Figure 25a. Prostate cancer screening as a function of SES and lifestyle while adjusting for all exogenous variables and assuming no covariation between SES and lifestyle

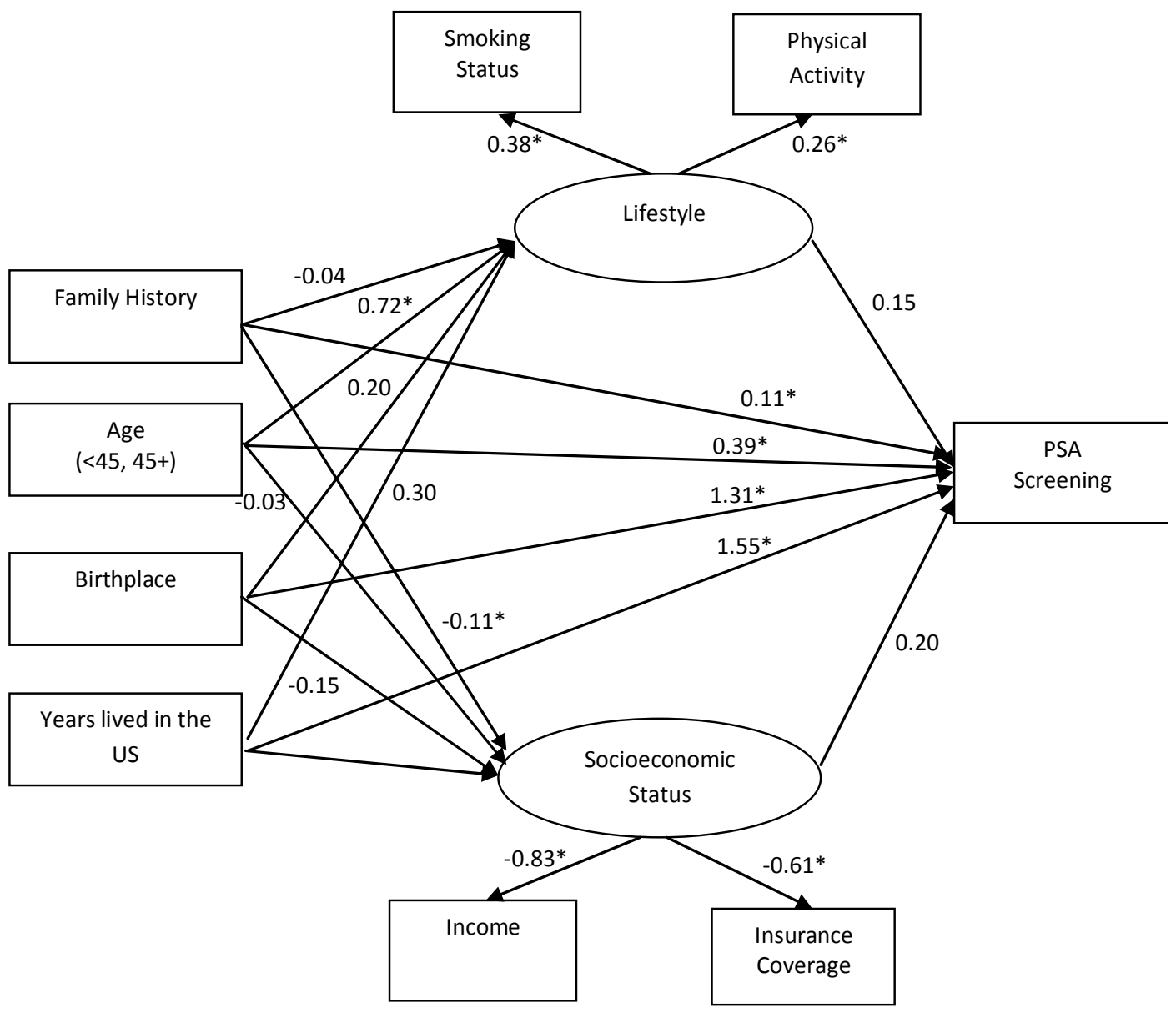

*Statistically significant path coefficient 
Table 22a. Structural equation model assessing the effect of both latent variables lifestyle and SES on prostate cancer screening while adjusting for family history of cancer, age group, birthplace, and years lived in the United States

\begin{tabular}{lrrrr}
\hline Indicator Variables & Estimate & S.E. & t value & $\begin{array}{r}\text { Two Tailed } \\
\text { p-value }\end{array}$ \\
\hline LS - Smoking status & & & & 0.000 \\
LS - Physical activity & 0.380 & 0.093 & 4.080 & 0.000 \\
LS - Family history & -0.258 & 0.071 & -3.659 & 0.589 \\
LS - Age $(<45,45+)$ & -0.039 & 0.072 & -0.541 & 0.000 \\
LS - Birthplace & 0.722 & 0.158 & 4.570 & 0.338 \\
LS - Years lived in the US & 0.201 & 0.210 & 0.958 & 0.116 \\
SES - Insurance & 0.300 & 0.191 & 1.570 & 0.000 \\
SES - Income & 0.608 & 0.041 & 14.938 & 0.000 \\
SES - Family history & -0.830 & 0.165 & -5.025 & 0.019 \\
SES - Age (<45, 45+) & -0.110 & 0.047 & -2.342 & 0.506 \\
SES - Birthplace & -0.033 & 0.049 & -0.665 & 0.997 \\
SES - Years lived in the US & -0.001 & 0.149 & -0.004 & 0.272 \\
PSA - Lifestyle & 0.151 & 0.137 & 1.099 & 0.475 \\
PSA - SES & 0.151 & 0.212 & 0.714 & 0.001 \\
PSA - Family history & -0.203 & 0.063 & -3.229 & 0.000 \\
PSA - Age (<45, 45+) & 0.107 & 0.028 & 3.820 & 0.014 \\
PSA - Birthplace & 0.392 & 0.159 & 2.459 & 0.000 \\
PSA - Years lived in the US & $1.314 \dagger$ & 0.107 & 12.311 & 0.000 \\
& $1.546 \dagger$ & 0.137 & 11.309 & \\
Measurement Models: Evaluation of goodness of fit (Coefficients) & & \\
Akaike (AIC) & & & & \\
$* *$ LS - Lifestyle & 9100.517 & & & \\
+ + Due to problems with convergence this value could not be accurately computed & \\
\hline
\end{tabular}

In this model, the path from age to lifestyle is significant with a positive relationship. For the latent variable SES, the path from family history of cancer is significant. Neither birthplace nor number of years lived in US have significant effects on both SES and lifestyle. Moreover, family history of cancer is statistically significant for explaining SES but non-significant for lifestyle.

Of all the direct effects to PSA screening, the path from lifestyle is the only one that is not significant. The path from SES to PSA has a negative coefficient indicating that as SES 
score, PSA score decreases, as observed earlier. The other significant paths (family history of cancer, age, birthplace and number of years lived in US) have positive correlations meaning as each indicator score increases the likelihood of PSA screening increases as well. The variables birthplace and number of years lived in the US however had problems converging and therefore did not produce reliable estimates. As a result Figure $25 \mathrm{~b}$ was assessed excluding years lived in the US. 
Figure 25b. Prostate cancer screening as a function of SES and lifestyle while adjusting for exogenous variables, excluding years in the US, and assuming no covariation between SES and lifestyle

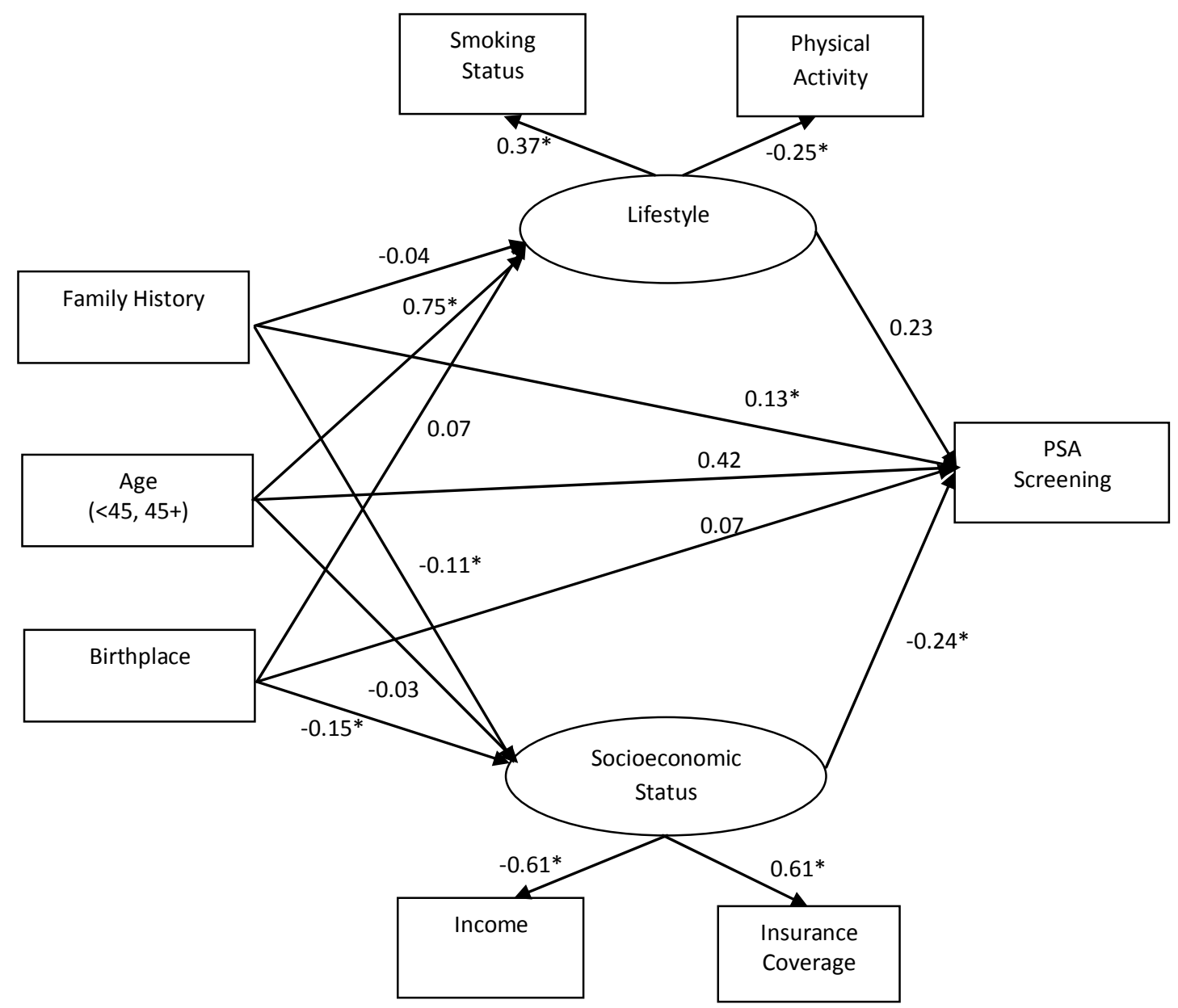

*Statistically significant path coefficient 
Table 22b. Structural equation model assessing the effect of both latent variables lifestyle and SES on prostate cancer screening while adjusting for family history, age group, and birthplace

\begin{tabular}{lrrrr}
\hline Indicator Variables & Estimate & S.E. & t value & $\begin{array}{r}\text { Two Tailed } \\
\text { p-value }\end{array}$ \\
\hline LS - Smoking status & & & & 0.003 \\
LS - Physical activity & 0.368 & 0.125 & 2.937 & 0.008 \\
LS - Family history & -0.251 & 0.094 & -2.657 & 0.578 \\
LS - Age (<45, 45+) & -0.042 & 0.075 & -0.557 & 0.002 \\
LS - Birthplace & 0.752 & 0.241 & 3.116 & 0.349 \\
SES - Insurance & -0.074 & 0.079 & -0.936 & 0.000 \\
SES - Income & 0.613 & 0.043 & 14.381 & 0.000 \\
SES - Family history & -0.826 & 0.178 & -4.633 & 0.019 \\
SES - Age (<45, 45+) & -0.110 & 0.047 & -2.338 & 0.536 \\
SES - Birthplace & -0.030 & 0.049 & -0.619 & 0.007 \\
PSA - Lifestyle & -0.145 & 0.054 & -2.696 & 0.532 \\
PSA - SES & 0.230 & 0.367 & 0.625 & 0.001 \\
PSA - Family history & -0.241 & 0.073 & -3.301 & 0.001 \\
PSA - Age (<45, 45+) & 0.128 & 0.038 & 3.380 & 0.188 \\
PSA - Birthplace & 0.422 & 0.321 & 1.316 & 0.080 \\
Measurement Models: Evaluation of goodness of fit (Coefficients) & & \\
Akaike (AIC) & & & & \\
$* *$ LS = Lifestyle & 9105.547 & & & \\
\hline
\end{tabular}

In this model, the path from family history of cancer and birthplace to latent variable lifestyle are not significant. Of the paths from exogenous variables to SES age group is not significant. The paths from family history and birthplace to SES are negative and significant. Of the direct paths to PSA, SES has an inverse significant relationship, birthplace and family history of cancer have a positive and significant relationship. Although the modification made for Figure 25b only resulted in an increase from 9100 (Figure 25a) to 9105 (Figure 25b) of the AIC, Figure $25 \mathrm{~b}$ is a better model because the problem of convergence has been corrected. 


\section{Partial mediation of the effects of exogenous variables with covariation between latent variables SES and lifestyle}

This model (Figure 26) was intended to assess the big picture putting together both measurement models for lifestyle and socioeconomic status to provide insight into how identified prostate cancer risk factors (family history of cancer, age group, birthplace, and years lived in the United States) as well as lifestyle and socioeconomic status impact prostate cancer screening behavior. Contrary to the previous models (Figure 25a, Figure 25b), covariation between both latent variables lifestyle and SES was allowed, along with the direct pathways of the exogenous variables to PSA. 
Figure 26. Prostate cancer screening as a function of SES and lifestyle with correlation between lifestyle and SES while adjusting for exogenous variables, excluding years lived in the US

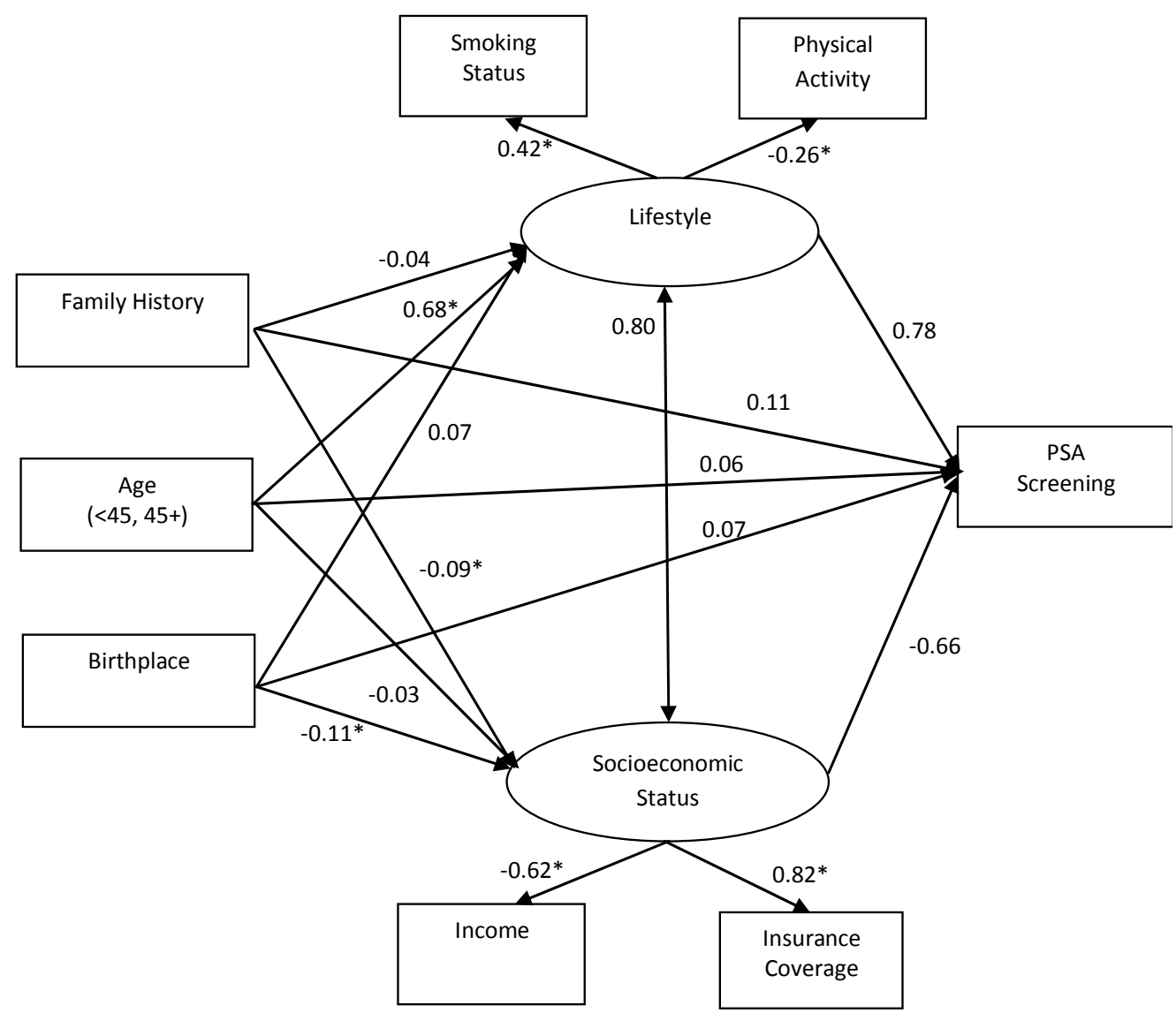

*Statistically significant path coefficient 
Table 23. Structural equation model assessing the effect of both latent variables lifestyle and SES on prostate cancer screening with covariation between latent variables SES and lifestyle while adjusting for family history, age group, birthplace, and years lived in the United States

\begin{tabular}{lrrrr}
\hline Indicator Variables & Estimate & S.E. & t value & $\begin{array}{r}\text { Two Tailed } \\
\text { p-value }\end{array}$ \\
\hline LS - Smoking status & & & & 0.000 \\
LS - Physical activity & 0.415 & 0.058 & 7.137 & 0.000 \\
LS - Family history & -0.260 & 0.051 & -5.126 & 0.591 \\
LS - Age (<45, 45+) & -0.037 & 0.068 & -0.538 & 0.000 \\
LS - Birthplace & 0.683 & 0.088 & 7.782 & 0.326 \\
SES - Insurance & -0.069 & 0.071 & -0.983 & 0.000 \\
SES - Income & 0.816 & 0.022 & 36.938 & 0.006 \\
SES - Family history & -0.621 & 0.225 & -2.761 & 0.037 \\
SES - Age (<45, 45+) & -0.090 & 0.043 & -2.087 & 0.546 \\
SES - Birthplace & -0.026 & 0.043 & -0.603 & 0.023 \\
PSA - Lifestyle & -0.112 & 0.049 & -2.277 & 0.474 \\
PSA - SES & 0.777 & 1.084 & 0.717 & 0.370 \\
PSA - Family history & -0.663 & 0.739 & -0.897 & 0.089 \\
PSA - Age (<45, 45+) & 0.116 & 0.068 & 1.703 & 0.314 \\
PSA - Birthplace & 0.056 & 0.750 & 0.074 & 0.264 \\
Covariation SES - Lifestyle & 0.066 & 0.065 & 1.008 & \\
Measurement Models: Evaluation of goodness of fit (Coefficients) & & \\
Akaike (AIC) & 0.795 & 0.712 & 1.117 & \\
** LS - Lifestyle & 9057.098 & & & \\
\hline
\end{tabular}

The results from this model show that the addition of the correlation between latent variables SES and lifestyle improved the model fit $(\mathrm{AIC}=9057.098$ versus 9105.547). This improvement in model fit came along with changes in the magnitude of the different pathways and the associated significance levels as shown in the tables above (Tables 22a-b \& 23).

In this full model the pathways to latent variable lifestyle were not significant for family history (negative effect) and birthplace (negative effect). The path between age group and lifestyle was significant and had a positive association, meaning that the $45+$ group of men is more likely to increase their scores of lifestyle compared to the men younger than 45 years of age. 
For the latent variable SES, all the pathways were negative, and only the relationship with exogenous variable age group was not statistically significant.

The correlation between latent variables lifestyle and SES is not significant. Lifestyle was positively associated with the PSA screening, although not significant. On the other hand, SES was negatively associated with PSA and not significant as well. Among the exogenous variables, family history, birthplace, and age group were positively associated with PSA screening although not significant.

\section{Structural equation model for the Caribbean-born group: Partial mediation of the effects of exogenous variables without covariation between latent variables SES and lifestyle}

The following model (Figure 27) focused on the effects of the latent variables SES and lifestyle on PSA screening among the Caribbean-born subgroups, while adjusting for exogenous variables (family history of cancer, age groups, Caribbean birthplace, as well as the number of years lived in the US). 
Figure 27. Prostate cancer screening as a function of latent variables SES and lifestyle while adjusting for exogenous variables among Caribbean-born men without covariation between latent variables SES and lifestyle

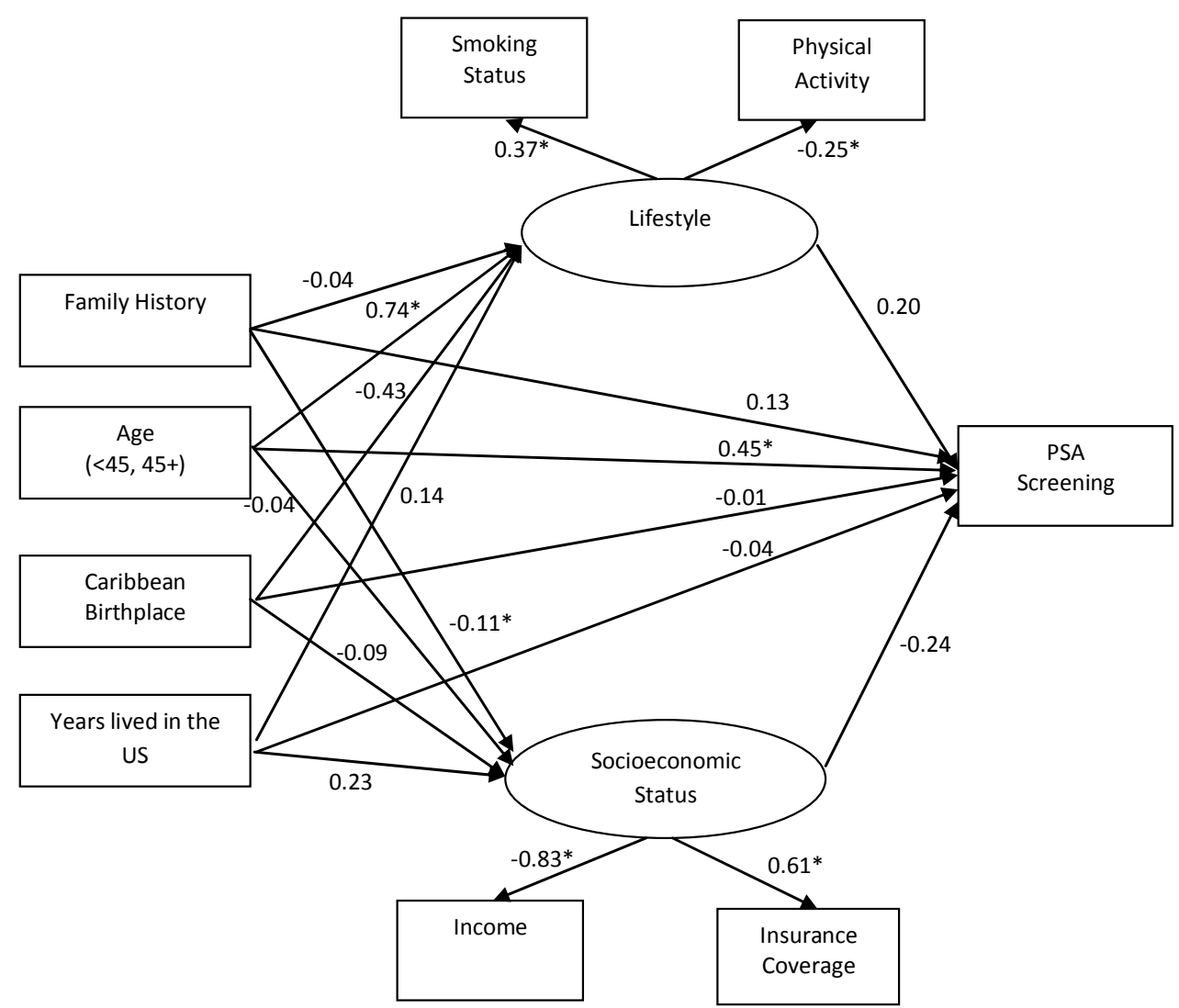

*Statistically significant path coefficient 
Table 24. Structural equation model of exogenous variables (family history of cancer, age group, Caribbean birthplace, and years lived in the United States), latent variables lifestyle and socioeconomic status, and PSA screening

\begin{tabular}{lrrrr}
\hline Indicator Variables & Estimate & S.E. & t value & $\begin{array}{r}\text { Two Tailed } \\
\text { p-value }\end{array}$ \\
\hline LS - Smoking status & & & & 0.000 \\
LS - Physical activity & 0.370 & 0.101 & 3.650 & 0.001 \\
LS - Family history & -0.254 & 0.077 & -3.294 & 0.584 \\
LS - Age $(<45,45+)$ & -0.041 & 0.074 & -0.548 & 0.000 \\
LS - Caribbean birthplace & 0.743 & 0.187 & 3.983 & 0.742 \\
LS - Years lived in the US & -0.043 & 0.132 & -0.329 & 0.248 \\
SES - Insurance & 0.143 & 0.124 & 1.156 & 0.000 \\
SES - Income & 0.608 & 0.042 & 14.455 & 0.000 \\
SES - Family history & -0.833 & 0.172 & -4.829 & 0.020 \\
SES - Age (<45, 45+) & -0.109 & 0.047 & -2.318 & 0.470 \\
SES - Caribbean birthplace & -0.035 & 0.049 & -0.722 & 0.537 \\
SES - Years lived in the US & -0.085 & 0.137 & -0.618 & 0.064 \\
PSA - Lifestyle & 0.225 & 0.122 & 1.850 & 0.468 \\
PSA - SES & 0.201 & 0.277 & 0.726 & 0.001 \\
PSA - Family history & -0.240 & 0.071 & -3.371 & 0.160 \\
PSA - Age (<45, 45+) & 0.126 & 0.034 & 3.662 & 0.050 \\
PSA - Caribbean birthplace & 0.448 & 0.228 & 1.960 & 0.621 \\
PSA - Years lived in the US & -0.013 & 0.027 & -0.494 & 0.412 \\
Measurement Models: Evaluation of goodness of fit (Coefficients) & & \\
Akaike (AIC) & -0.043 & 0.052 & -0.821 & \\
** LS = Lifestyle & & & & \\
\hline
\end{tabular}

As in previous models, exogenous variables were allowed to have both direct pathways to PSA as well as indirect pathways to PSA through both latent variables SES and lifestyle.

Examining direct paths to PSA screening, results (Table 24) show negative effects of the latent variable SES, Caribbean birthplace and the number of years lived in the US as well as positive effect of age group. However, only the effects of SES and age group (marginally) were statistically significant. On the other hand, effects of latent variable lifestyle, and family history of cancer, were positive but non-significant. Although the path from family history to PSA screening was not significant, the odds of PSA screening for men with a family history of cancer were 2.2 times compared to men with no family history of cancer. As expected, men in the 45+ 
age group were 10.3 times more likely to undergo PSA screening compared to the younger group (age $<45)$.

Examining all pathways from exogenous variables to both latent variables SES and lifestyle, results show that age group effects on lifestyle were positive and significant, while effects of family history and Caribbean birthplace were negative and non-significant (Table 24). As for the associations between the latent variable SES and exogenous variables, family history, age group, and Caribbean birthplace were negatively associated with the latent SES variable, and the association was statistically significant only for family history of cancer. The number of years lived in the US was the only exogenous variable positively affecting latent variable SES scores but its significance was borderline (p-value 0.06) (Table 24).

Compared to the whole group (all non-Hispanic Black men), the Caribbean-born group does not differ much in the comparison with US-born Black men in some regards. Indeed, for the path from SES to PSA associations are quite similar, parameter estimates: $-0.203,-0.240$; pvalue: $0.001,0.001$, for the whole group and Caribbean-born men respectively (Tables $22 \& 24$ ). The path comparisons for the whole group (US-born versus Caribbean-born) variable birthplace and Caribbean-born (all Caribbean islanders) variable Caribbean birthplace effects on SES and lifestyle latent variables were not statistically significant.

\section{Structural equation model for the Caribbean-born group: Partial mediation of the effects of exogenous variables with covariation between latent variables SES and lifestyle}

In this model (Figure 28), the covariation between both latent variables lifestyle and SES was added). Improvement of the model fit was assessed using the Akaike Information Criteria 
(AIC), smaller values of AIC leading to a better fitting model, when comparing different models. The model results are presented in Table 28 below.

Results show a statistically non-significant covariation between both latent variables SES and lifestyle $(\mathrm{p}=0.263)$. Although non-significant, the covariation between both latent variables SES and lifestyle improved the model fit as shown by their respective AIC values (AIC $=$ 9059.932 versus 9107.906). Covariation between the latent variables also changed the relationships between PSA screening and some of the other variables in the model. The direct pathways to PSA from latent variables SES and lifestyle, although non-significant, increased in absolute value (lifestyle: stronger positive association, SES: stronger negative association), while the direct pathway from age group decreased drastically, odds ratio dropping from 10.34 (statistically significant) to 1.13 (statistically non-significant). The effects of family history of cancer as well as Caribbean birthplace remained almost the same $(\mathrm{OR}=2.209$ compared to $\mathrm{OR}=2.179$, and $\mathrm{OR}=0.948$ compared to $\mathrm{OR}=0.970$, respectively for family history of cancer and Caribbean birthplace).

Among the paths from exogenous variables to the latent variable lifestyle, both age group and years lived in the US showed positive coefficients but age group was the only one to have a significant path. Family history of cancer and Caribbean birthplace, on the other hand, showed negative effects on lifestyle but those effects were not statistically significant. All the relationships between latent variable lifestyle and exogenous variables decreased slightly in absolute value with the inclusion of the covariation between the latent variables.

For latent variable SES, there were negative effects of family history of cancer, age group and Caribbean birthplace but only the path from family history was significant. Among the 
effects from each of the exogenous variables and the latent variable SES, years lived in the US was the only variable positively associated with SES, the path was not statistically significant however. As for latent variable lifestyle, all the relationships between SES and exogenous variables decreased slightly in absolute value with the inclusion of the covariation. 
Figure 28. Prostate cancer screening as a function of SES and lifestyle while adjusting for exogenous variables within the Caribbean-born group with covariation between latent variables

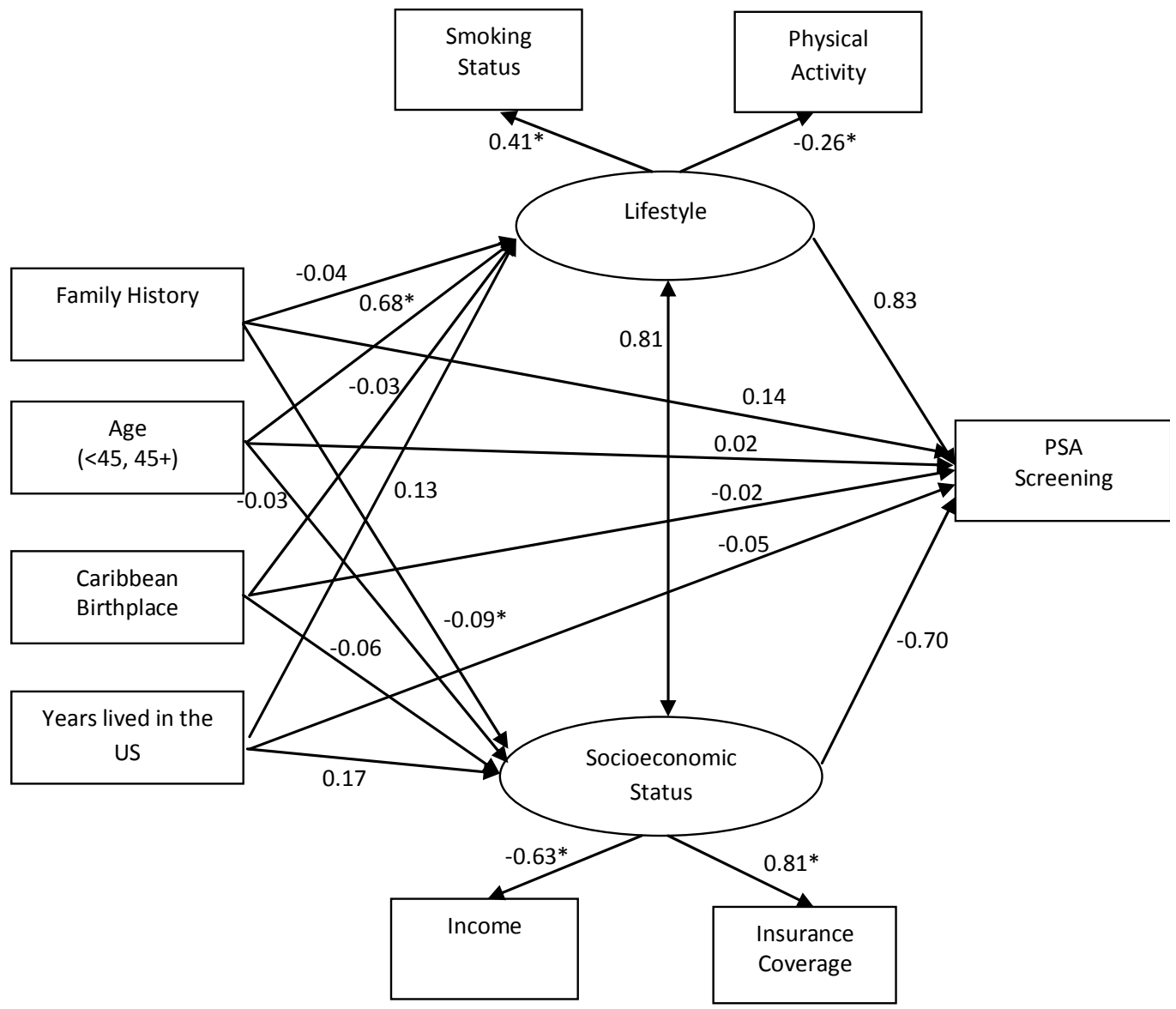

*Statistically significant path coefficient 
Table 25. Structural equation model assessing the effect of both latent variables lifestyle and SES on prostate cancer screening with a correlation between SES and lifestyle while adjusting for family history of cancer, age group, Caribbean birthplace, and years lived in the United States

\begin{tabular}{lrrrr}
\hline Indicator Variables & Estimate & S.E. & t value & $\begin{array}{r}\text { Two Tailed } \\
\text { p-value }\end{array}$ \\
\hline LS - Smoking status & & & & 0.000 \\
LS - Physical activity & 0.414 & 0.058 & 7.145 & 0.000 \\
LS - Family history & -0.258 & 0.050 & -5.136 & 0.600 \\
LS - Age $(<45,45+)$ & -0.036 & 0.068 & -0.525 & 0.000 \\
LS - Caribbean birthplace & 0.681 & 0.088 & 7.763 & 0.787 \\
LS - Years lived in the US & -0.034 & 0.124 & -0.271 & 0.258 \\
SES - Insurance & 0.129 & 0.144 & 1.131 & 0.000 \\
SES - Income & 0.810 & 0.022 & 37.036 & 0.003 \\
SES - Family history & -0.626 & 0.213 & -2.938 & 0.036 \\
SES - Age (<45, 45+) & -0.089 & 0.043 & -2.095 & 0.482 \\
SES - Caribbean birthplace & -0.030 & 0.043 & 0.703 & 0.577 \\
SES - Years lived in the US & -0.063 & 0.114 & 0.558 & 0.102 \\
PSA - Lifestyle & 0.172 & 0.105 & 1.637 & 0.491 \\
PSA - SES & 0.830 & 1.204 & 0.689 & 0.383 \\
PSA - Family history & -0.700 & 0.803 & -0.872 & 0.109 \\
PSA - Age (<45, 45+) & 0.144 & 0.071 & 1.602 & 0.980 \\
PSA - Caribbean birthplace & 0.020 & 0.820 & 0.025 & 0.855 \\
PSA - Years lived in the US & -0.021 & 0.115 & -0.183 & 0.652 \\
Covariation SES - Lifestyle & -0.052 & 0.115 & -0.452 & 0.263 \\
& 0.805 & 0.720 & 1.119 & \\
Measurement Models: Evaluation of goodness of fit (Coefficients) & & \\
Akaike (AIC) & 9059.932 & & & \\
$* *$ LS = Lifestyle & & & & \\
\hline
\end{tabular}

The results for Figure 28 and Table 25 show that among the paths from the exogenous variables to latent variable lifestyle, age group is the only variable with a significant path to lifestyle. For latent variable SES however, the only significant path to SES from the exogenous variables is from family history of cancer which has a negative association. Among the direct effects from each of the exogenous variables and each latent variable to PSA screening none of the paths were significant. 
The models including the larger group of men, all non-Hispanic Black men, as well as the models for the Caribbean-born men only, the inclusion of the covariation between the latent variables SES and lifestyle in the models improved the model fit as measured by the AIC, although the improvement in both cases is as low as $0.5 \%$ change in AIC. Moreover, the inclusion of the covariation increased, in absolute value, some of the direct pathways to PSA (especially from latent variables SES and lifestyle). However, the estimates of the standard errors (SE) increased as well leading to low and non-significant t values. Given these facts, the models without covariation for both the larger group (non-Hispanic Black men) (Figure $25 \&$ Table 22) and the Caribbean-born group (Figure $27 \&$ Table24) are recommended. 


\section{CHAPTER V. \\ DISCUSSION}

\section{Overview}

Prostate cancer is the most common non-skin cancer among men in the United States. It is well established that African-American men are diagnosed more often than any other race and they often present at later stages $[3,10]$. Delays in diagnoses lead to poor outcomes (morbidity and mortality) that early diagnosis could avoid. Reported rates of prostate cancer incidence among Black men living in the Caribbean raised concerns this study aimed to address $[15,28$, 32]. The present study aimed to explore possible differences in prostate cancer prevalence and screening behavior by race/ethnicity and by country of birth among non-Hispanic Black men.

Immigrants in the United States represent a significantly large group that continues to grow. Foreign-born individuals accounted for $12.4 \%$ of the population in the US between 2005 2009 and Caribbean-born individuals accounted for $9 \%$ of the foreign-born group [73]. Immigrant groups tend to cluster in certain geographies and without a good understanding of their health needs, this group may not only suffer poorer health outcomes than their US-born counterparts but could possibly contribute to the burden on the health care system because of their unmet health care needs [74-76]. According to US census data from 2005-2009, Caribbean-born individuals tended to live in either the Northeast (47\%) or in the Southern region of the US (48\%), more specifically New York (29\%) and Florida (38\%) [73].

Generally, risk factors for cancer and more specifically prostate cancer include family history of cancer, age, and Black race $[44,54,77,78]$. The literature suggests that although the rate of prostate cancer in the US is highest among African-American men, published rates among 
men living in the Caribbean are even higher $[14,32,79]$. This raised the question of whether or not men born in the Caribbean and living in the United States, have a higher risk of prostate cancer than US-born Black men. If so, they should be screened as aggressively as, or more aggressively than, US-born Black men. Other factors such as increased age and a family history of cancer should increase the probability of being screened for cancer since these are wellestablished risk factors for prostate cancer and should raise awareness of the risk of disease [44, 80]. The results of this study however, did not provide a clear answer to this question of variation of prostate cancer prevalence and receipt of a PSA exam by birthplace.

\section{Prostate cancer prevalence trends}

Most studies that describe prostate cancer prevalence tend to stratify by race/ ethnicity. Studies such as Haas et al. (1997), Andriole et al. (2005), Abe et al. (2006) highlight important differences in prostate cancer incidence, prevalence, and survival by traditional race/ethnicity categories [81-83]. Not much information is in the literature on within group variance, especially

among non-Hispanic Black men. Research is more recently beginning to highlight the value of assessing within group variance among non-Hispanic Black men [19].

\section{Prevalence of prostate cancer}

Over half (55\%) of the non-Hispanic Black men who had been diagnosed with cancer reported prostate cancer as at least one of their cancer diagnoses while only $24 \%$ of non-Hispanic White men who had been diagnosed with cancer reported prostate cancer as at least one of their prostate cancer diagnoses. This data illustrates the differences in cancer burden by race. This shows that the probability of a prostate cancer diagnosis among non-Hispanic Black men is twice that of non-Hispanic White men when there is a cancer diagnosis. The overall prevalence of prostate cancer by race in this 10-year sample shows a higher trend among non-Hispanic Black 
men than non-Hispanic White men, which is consistent with the literature $[3,15,16]$. Although this trend was consistent with the literature, the magnitude of the difference in prevalence by race seen in the literature was not evident in this pooled sample. The differences between the groups, adjusted for survey year, were not statistically significant overall nor were they significant when comparing the trends over time.

The results of the pooled study sample show that Caribbean-born Black men reported a higher proportion of prostate cancer among cancer diagnoses than non-Hispanic Black men overall. When Caribbean-born men were stratified by survey year however, the sample sizes were too small to provide stable estimates. As a result it was not possible to draw solid conclusions based on the analysis to address Aims $1 \mathrm{~b}$ and 1c. For the entire 10-year sample overall, similar trends to what is suggested in the literature were observed in that Caribbean-born men tend to experience higher rates of prostate cancer [28, 84]. Although the sample size among the Caribbean-born participants is small and the data are to be interpreted with caution these results can be an indication that the magnitude of the problem is in fact greater among Caribbeanborn men than among US-born Black men.

Smith et al., summarized the American Cancer Society, cancer (all types) screening guidelines, which focused on variations in cancer risk and screening recommendations including differences by race/ethnicity [43]. A study by Gwede et al. (2010), stratified the study population into three ethnic groups (African-American, English-speaking Caribbean-born, and Haitian) which allowed for the exploration of within group variance in cancer screening behavior. The authors of this study found that there were in fact differences between the groups in terms of colorectal cancer screening knowledge and screening behavior [19]. 


\section{Risk factors associated with prostate cancer screening behavior}

To assess the effects of risk factors associated with prostate cancer screening behavior among non-Hispanic Black men, various structural equations models (SEM) were fitted to the data and compared. Two SEM models relating SES to PSA while adjusting for risk factors (all men: Figure 16 \& Table 13; Caribbean-born men: Figure $19 \&$ Table 16), two models relating lifestyle to PSA while adjusting for risk factors (all men: Figure 22 \& Table 19; Caribbean-born men: Figure $24 \&$ Table 21), and two models relating both SES and lifestyle to PSA while adjusting for risk factors (all men: Figure 25a \& Table 22a; Caribbean-born group: Figure 27 \& Table 24), represented the best choices of models, among all the SEM models considered for all the men in the sample and for Caribbean-born men respectively. These models were chosen based on both the AIC criteria and the fact that the convergence was perfectly reached. These models allowed both direct and indirect pathways of exogenous variables to PSA (the indirect pathways going through either SES or lifestyle or both).

\section{Socioeconomic status}

Socioeconomic status is thought to be a good determinant of health behavior and health care utilization $[53,85]$. Those who are of a higher socioeconomic status are more likely to be insured and therefore have more health care provider encounters than someone of a lower socioeconomic status [75]. This was an important factor to explore among this group of men because prostate cancer screening is an elective procedure and without health insurance this can be costly with no directly perceived benefit (cancer free test result). Women of a higher socioeconomic status are diagnosed with breast cancer more often, but these women, however, are diagnosed at earlier stages and have better survival rates than women of lower SES [53]. This trend can be attributed to breast cancer screening activity as more health care provider encounters and a higher rate of participation in screening increases the probability of early detection[86]. 
This relationship, however, was not as clear among men in the general population who have been diagnosed with prostate cancer. Our study results show an inverse relationship between SES and prostate cancer screening (receipt of the PSA exam). The results from the men in this NHIS survey sample show that men with higher SES are less likely to have had a PSA examination. This relationship was statistically significant in every model (with and without exogenous variables) when looking at all non-Hispanic Black men and among Caribbean-born men. These results indicate that unlike women, as SES increases among men, they are less likely to have PSA exam. This relationship is very clear in the models of complete mediation which is intended to illustrate the impact of SES on access to care, taking into consideration the selected risk factors. This finding is important when considering programs to increase screening among men. Here it shows that men of higher SES with the risk factors for prostate cancer should be targeted for health promotion activity related to receipt of a PSA exam. In the case of partial mediation the models allowed for the assessment of each individual risk factor on PSA screening while controlling for all other factors in the model. Again, the results of partial mediation show the same relationship of SES to PSA screening but the magnitude decreased as other factors contributed to the outcome of interest. Insurance status was broken up into two groups: a) uninsured, self pay; b) insured, which included private insurance, Medicare, and Medicaid. Although Medicaid and Medicare generally include persons with lower or fixed income this group made up a small portion of this study sample which may have an impact on the results; however, it is not likely given the small number. Both payer types were grouped in order to assess payment for preventive care as a barrier.

\section{Lifestyle}

Lifestyle in this analysis was different than SES in that the relationship between lifestyle and PSA screening wasn't as stable as it was for SES. Lifestyle as a complete mediator for risk 
factors (family history of cancer, age, years lived in US and birthplace) showed a stronger relationship with PSA screening than lifestyle alone, although the one risk factor, years lived in the US, was eventually excluded from the model due to unstable estimates. This is an interesting finding in that the literature suggests that years lived in the US is a strong predictor of health seeking behavior and should therefore impact preventive care, including PSA screening [40]. It is possible that there wasn't enough variation in the years lived in the United States among the immigrants included in this sample. Partial mediation dramatically reduced the magnitude of the relationship between lifestyle and PSA screening and indicated that age is a strong determinant of PSA screening, which is consistent with what is expected.

\section{Smoking as a risk factor}

The smoking variable highlighted interesting facts about this population. Non-Hispanic White men were the most likely to report ever being smokers, while Caribbean-born men were the least likely to report ever being smokers (Table 1). Taking into consideration the global trends of smoking reported by the World Health Organization (WHO) one would expect for Caribbean-born men and US-born men to report similar rates of smoking [87]. Instead, among all men in this study sample, non-Hispanic White men reported a rate of ever smoking that was twice that of the 2006 WHO prevalence (53\% vs. 25\%). Caribbean-born men reported a rate of $25 \%$, which was more in line with the average prevalence reported by the WHO [87]. The WHO estimates that the prevalence of smoking in countries like St. Lucia, Jamaica, and Barbados are $28 \%, 21 \%$, and $18 \%$ respectively [87]. Studies often report that immigrant groups are less likely to smoke than non-Hispanic Whites, which is consistent with the results of this NHIS study population [88]. One factor to consider in the prevalence of men who reported ever smoking is that there is a different age distribution between groups with non-Hispanic White men being slightly older. This difference in the age distribution may be a contributing factor to the number 
of men reporting ever having smoked or there could be differences in cultural norms. Reportedly, in developing countries like the Caribbean, more men die from tobacco related diseases than in developed countries like the US [3]. The lower rates of smoking could however be due to underreporting by immigrant groups. This idea is supported by a study done in Italy which found that although reported smoking rates have been declining over time, cigarette consumption measured by sales did not reflect the same decline [89]. Among men diagnosed with cancer however, a larger proportion (64\%) of these men reported ever smoking. Over 70\% of non-Hispanic Black men were ever smokers compared to over $40 \%$ of Caribbean-born black men in this NHIS pooled study sample.

\section{Age group as a risk factor}

The age distribution among survey participants is similar to what is seen in the general population. In this study sample both the non-Hispanic Black men and the Hispanic men were younger than non-Hispanic White men. Caribbean born Black men were younger than US born black men which was expected as immigrants tend to be younger [76]. Okie (2007) suggests that immigrants tend to be younger and healthier because younger people tend to migrate to the US for employment opportunities [76]. In each of the models considered in this study, age group $(<45,45+)$ showed a strong impact on the receipt of the PSA exam. In the case of complete mediation models, the paths from age to latent variables SES and lifestyle were generally significant. For partial mediation models, however, the indirect paths to PSA exam (through SES or lifestyle) were reduced while the direct paths to PSA exam would generally be increased and statistically significant. This is interesting as it is expected that age should impact the probability of being screened since prostate cancer is a disease of the ageing and screening for prostate cancer is therefore recommended for older men. It was expected however that age would also 
have a strong and positive relationship with both SES and lifestyle which would in turn positively impact PSA screening.

\section{Immigrant characteristics}

The literature also suggests that the majority of immigrants tend to be laborers and tend to work for employers that do not provide insurance for their employees [74-76, 90, 91]. The majority $(>75 \%)$ of the men in this study sample were insured however, and almost $100 \%$ of the men diagnosed with prostate cancer in this study sample were insured. It is expected that those who are insured have better access to care and are more likely to engage in primary preventive care. The insured have more interactions with their physician, are more likely to have a regular doctor and are therefore more likely to be screened which increases the probability of being diagnosed [74]. So many of the men in this study sample are insured, which is different than what was expected. This could be an indication that Caribbean-born men are different from other immigrant groups in that the majority of Caribbean-born men are in fact insured. Another possible explanation for the difference seen among the Caribbean-born survey participants could also be that the type of person who would agree to participate in the National Health Interview Survey is different than others with similar demographic characteristics. It is possible that those who are likely to be uninsured or have questionable immigration status may be less likely to participate in this type of survey and therefore capturing their health behavior and/or their health status would not be possible.

\section{Caribbean birthplace}

\section{Overall characteristics}

Among Caribbean born men, like other groups, health seeking behavior is not attributed to one factor in isolation. The structural equation models sought to explore the complete 
environment taking into account cancer risk factors and their relationship with having a PSA exam. In the SEM models we found that SES had a significant and inverse relationship with the receipt of a PSA exam which is opposite of what was expected. This could be an indication that as SES increases among men, the probability of the receipt of a PSA exam as recommended actually decreases. This can be attributed to many factors, such as busy schedule for high income individuals, resistance to pay for a service whose benefits are not clearly seen, low levels of perceived benefits of PSA screening, and other male-driven perceptions of the screening in general. Another probable explanation is that SES as defined by insurance and income may not have reflected SES as expected.

The SEM models showed that lifestyle had a positive but non-significant effect on the likelihood of PSA screening. Moreover, there were non-significant effects of the Caribbeanspecific birthplace on PSA screening.

Years lived in the US

Years lived in the United States is often used as a proxy for acculturation, which has been shown to impact health seeking behavior [19]. Studies show that the longer immigrants live in their host county, the more likely they are to engage in primary prevention activities such as cancer screening $[19,92]$. The men in this NHIS sample however, did not seem to follow this trend. In fact, the number of years lived in the US did not overall consistently have a positive, nor significant impact on the receipt of a PSA exam. The effects were rather weak, nonsignificant and opposite to what were expected.

\section{Study limitations}

There are several limitations to take into consideration when using a national health database. Primarily one must consider that all data collected is self reported data. Self reported 
survey data are subject to recall bias, selection bias, and differential reporting depending on the sensitivity of the subject matter.

The National Health Interview Survey is a voluntary survey. Not all people are comfortable with providing personal information for this type of survey, although anonymous. The type of person who chooses to participate may differ from those who refuse to participate lending toward inherent selection bias. This is especially important among immigrants (Caribbean-born) who could potentially have questionable immigration status. These people may be less likely to participate as they may fear negative impacts on their lives.

The sample design used by NHIS is an additional limitation when trying to evaluate the Caribbean-born immigrant population. Immigrants are known to cluster in certain geographic areas and although the NHIS sample design oversamples Blacks they are not able to capture a large number of Caribbean born individuals if they are not oversampling areas with a heavy concentration of Caribbean-born individuals. This is apparent in small sample size of Caribbeanborn individuals this 10-year pooled sample of Black men. The current sampling plan did not capture enough Caribbean-born individuals for meaningful stratified analysis over time. This led to the problem of unreliable estimates for these small groups.

An important factor to consider is the potential for recall bias as the analysis in this study focused on questions requiring the survey participant to recall ever having a PSA exam, ever being diagnosed with cancer or prostate cancer. Without confirmation by review of the medical records of the respondent there is the potential for misinformation. A potential problem with accurately capturing a history of a PSA exam is that it is a blood test. They may understand it to be a part of a battery of standard blood tests and may not be familiar with the terminology 
associated with the blood test for prostate cancer. This may impact accurate recall as men may not remember that they had this specific test done. The use of the PSA test has allowed for detection of prostate cancer earlier than would be detected with other methods such as the DRE. This method however is not perfect in that there's no definitive marker for what is considered elevated which highlights the importance of baseline numbers to be used as a comparator [3]. The nature of prostate cancer can also complicate the use of the PSA exam. In some instances prostate cancer is a very slow growing disease which may never impact the man's health and diagnosis of this form of prostate cancer may lead to undue worry and unnecessary treatment.

Self reported data is vulnerable to missing data that is not at random. Some questions may be disproportionately impacted depending on the sensitivity of the information. Questions of a sensitive nature may be less likely to be answered at all or answered truthfully. This includes questions about lifestyle factors, like ever smoked. There is the potential for answering these types of questions in a way to seem favorable rather than reflecting the actual scenario.

A potential limitation includes the coding of insurance status. All survey participants reporting a payer (private, Medicaid, Medicare) for their health care were coded as insured. There is a potential impact on the interpretation of this data as the inclusion of men covered by Medicare and Medicaid may impact study results. The population covered by government sponsored programs may differ from those with private insurance coverage in terms of socioeconomics, health status, and age. The thought behind coding this way was that since the goal was to look at screening behavior it was more important to look at self pay versus other rather than to stratify by the type of payer in terms of payment for preventive care being a potential barrier. 
National survey data provide information cross sectional data which potentially capture incidence and prevalence data simultaneously. This is important as this study classified all reported prostate cancer diagnoses as prevalent and did not capture date of diagnosis. There are potential impacts to the interpretation of health seeking behavior when considering incident versus prevalent cases of prostate cancer. Men who were diagnosed with prostate cancer via DRE and were treated prior to the uptake of the PSA exam would be categorized as unscreened. 


\section{CHAPTER VI.}

\section{CONCLUSIONS}

The results of this study show that when non-Hispanic Black men are stratified by birthplace, Caribbean-born Black men had a higher overall prevalence of prostate cancer diagnoses than US-born Black men 40/1000 vs. 37/1000 respectively. This information is of value in that the high prevalence reported in the Caribbean persists among Caribbean-born men living in the United States. Additionally, among the men in this NHIS sample, there was not statistically significant difference in the prevalence of prostate cancer between non-Hispanic Black men and non-Hispanic White men over time (1999-2008). This finding is very different from existing trends in the literature.

To assess differences in prostate cancer screening behavior among non-Hispanic Black men, various structural equations models were fitted to the data. Factors impacting cancer screening behavior are numerous, however the key factors (lifestyle, SES, family history of cancer, age group, birthplace) selected for the SEM modeling in this study allowed for an indepth assessment of the differences in the screening experience of the men in the study sample. The results of this study found that there are differences in the screening behavior of US-born Black men versus Caribbean-born Black men as observed in the various SEM models (especially in partial mediation models SES-PSA, Lifestyle-PSA, and the complete model SES-lifestylePSA). Indeed, from the best models retained and considering only the direct effects of birthplace to PSA, US-born men were 2 to 3 times more likely to undergo PSA screening test compared to Caribbean-born men. However, among the Caribbean islanders, there were no significant differences in their screening behaviors. 
Differences were also found in the screening experience among non-Hispanic Black men, with respect to their SES and lifestyle, when taking into consideration all risk factors specified in the models. As SES increased, non-Hispanic Black men were 1.5 to 1.6 times more likely to not undergo PSA screening. On the other hand, as their lifestyle score increased, they were 1.1 to 2.3 times more likely to undergo PSA screening, although these differences were not statistically significant. Among the other risk factors, significant differences were observed with respect to family history of cancer. Men with a positive family history of cancer were 2.0 to 2.3 times more likely to undergo PSA screening exams compared to those without a family history of cancer.

Although limitations in the sample size of the Caribbean-born group did not allow for conclusive analysis of trends over time and limited the amount of stratified analyses performed, the results are important in that they show evidence of a difference in the reported prevalence of prostate cancer and also shows that there are differences in screening behavior between US-born and Caribbean-born men. This information is useful in showing a gap in research in the need for further exploration of differences that exist among immigrant groups. Further research is warranted into the health care needs, more specifically as they pertain to prostate cancer, of Caribbean-born Black men. The potential impact of different social and cultural norms should be further explored to reduce the probability of mortality related to prostate cancer, which when caught early has a favorable survival rate.

This study only scratches the surface in providing information on prostate cancer among Caribbean-born men. One potential improvement in the ability to monitor and positively impact this population would be for national health surveys such as NHIS, to take steps to obtain large enough samples annually in order to provide researchers with enough data for complex in-depth analysis. Another potential activity that could positively impact future research, allowing more 
comprehensive assessment of this population, would be to put greater emphasis on capturing country of birth, in medical records and in cancer registries, so that people can be correctly classified when reporting health outcomes such as cancer diagnoses. These steps would be a great contribution in allowing for more informed decision making when attempting to address the health care needs of Caribbean-born immigrants through program planning and/or resource allocation. 


\section{REFERENCES}

1. NCI, US. National Institutes of Health. Prostate Cancer: Prevention. 2007.

2. Weinrich, S.P., Prostate cancer screening in high-risk men: African American Hereditary Prostate Cancer Study Network. Cancer, 2006. 106(4): p. 796-803.

3. ACS, Cancer Facts \& Figures 2010. 2010, American Cancer Society: Atlanta.

4. ACS, Cancer Facts \& Figures 2011. 2011, American Cancer Society: Atlanta.

5. ACS, Cancer Facts \& Figures 2009. 2009, American Cancer Society: Atlanta.

6. ACS, Cancer Facts \& Figures 2008. 2008, American Cancer Society: Atlanta.

7. Kushi, L.H., et al., American Cancer Society Guidelines on Nutrition and Physical Activity for cancer prevention: reducing the risk of cancer with healthy food choices and physical activity. CA Cancer J Clin, 2006. 56(5): p. 254-81; quiz 313-4.

8. Blocker, D.E., et al., Knowledge, beliefs and barriers associated with prostate cancer prevention and screening behaviors among African-American men. J Natl Med Assoc, 2006. 98(8): p. 1286-95.

9. Williams, H. and I.J. Powell, Epidemiology, pathology, and genetics of prostate cancer among African Americans compared with other ethnicities. Methods Mol Biol, 2009. 472: p. 439-53.

10. Jones, R.A., R. Steeves, and I. Williams, How African American men decide whether or not to get prostate cancer screening. Cancer Nurs, 2009. 32(2): p. 166-72.

11. Joseph, H.J., Determinants of prostate cancer screening in a sample of African American military servicemen. Mil Med, 2006. 171(5): p. 430-5.

12. Peek, M.E. and J.H. Han, Disparities in screening mammography. Current status, interventions and implications. J Gen Intern Med, 2004. 19(2): p. 184-94.

13. Oliver, S.E., M.T. May, and D. Gunnell, International trends in prostate-cancer mortality in the "PSA ERA". Int J Cancer, 2001. 92(6): p. 893-8.

14. Gonzalez, J.R., et al., Barriers to the initiation and maintenance of prostate specific antigen screening in Black American and Afro-Caribbean men. J Urol, 2008. 180(6): p. 2403-8; discussion 2408.

15. Consedine, N.S., et al., Fear and loathing in the Caribbean: three studies of fear and cancer screening in Brooklyn's immigrant Caribbean subpopulations. Infect Agent Cancer, 2009. 4 Suppl 1: p. S14.

16. Tewari, A.K., et al., Effect of socioeconomic factors on long-term mortality in men with clinically localized prostate cancer. Urology, 2009. 73(3): p. 624-30. 
17. Sloane, D., Cancer epidemiology in the United States: racial, social, and economic factors. Methods Mol Biol, 2009. 471: p. 65-83.

18. Greenlee, R.T. and H.L. Howe, County-level poverty and distant stage cancer in the United States. Cancer Causes Control, 2009.

19. Gwede, C.K., et al., Exploring disparities and variability in perceptions and self-reported colorectal cancer screening among three ethnic subgroups of U. S. Blacks. Oncol Nurs Forum, 2010. 37(5): p. 581-91.

20. Ben-Shlomo, Y., et al., The risk of prostate cancer amongst black men in the United Kingdom: the PROCESS cohort study. Eur Urol, 2008. 53(1): p. 99-105.

21. Kagotho, N. and J. Tan, Predictors of prostate cancer screening among older immigrant men. J Natl Med Assoc, 2008. 100(10): p. 1168-74.

22. Best Plummer, W.S., P. Persaud, and P.J. Layne, Ethnicity and cancer in Guyana, South America. Infect Agent Cancer, 2009. 4 Suppl 1: p. S7.

23. Wolf, M.S., et al., Literacy, race, and PSA level among low-income men newly diagnosed with prostate cancer. Urology, 2006. 68(1): p. 89-93.

24. Hoffman, R.M., et al., Racial and ethnic differences in advanced-stage prostate cancer: the Prostate Cancer Outcomes Study. J Natl Cancer Inst, 2001. 93(5): p. 388-95.

25. Adlercreutz, H., et al., Phytoestrogens and prostate disease. J Nutr, 2000. 130(3): p. 658S-9S.

26. ACS, Cancer Facts \& Figures 2007. 2007, American Cancer Society: Atlanta.

27. Kramer, B.S., et al., Prostate cancer screening: what we know and what we need to know. Ann Intern Med, 1993. 119(9): p. 914-23.

28. Bunker, C.H., et al., Prostate cancer screening parameters in a high-risk AfricanCaribbean population. Urology, 2004. 63(4): p. 737-41.

29. Chen, A.C., et al., Prostate-specific antigen, sex steroid hormones, and the insulin-like growth factor axis in U.S.-born, Jamaican, and Haitian black men: a pilot study. Urology, 2004. 64(3): p. 522-7.

30. Freeman, V.L., J. Leszczak, and R.S. Cooper, Race and the histologic grade of prostate cancer. Prostate, 1997. 30(2): p. 79-84.

31. Fowler, J.E., Jr. and S.A. Bigler, A prospective study of the serum prostate specific antigen concentrations and Gleason histologic scores of black and white men with prostate carcinoma. Cancer, 1999. 86(5): p. 836-41.

32. Glover, F.E., Jr., et al., The epidemiology of prostate cancer in Jamaica. J Urol, 1998. 159(6): p. 1984-6; discussion 1986-7. 
33. Helzlsouer, K.J., et al., Association between alpha-tocopherol, gamma-tocopherol, selenium, and subsequent prostate cancer. J Natl Cancer Inst, 2000. 92(24): p. 2018-23.

34. Papadopoulos, R., Lees, S. Cancer and culture: Investigating meaning and experiences of cancer of men from different ethnic groups: A pilot study. 2002 [cited 2004 November 7]; Available from: http://www.mdx.ac.uk/www/rctsh/cancer\&culture.pdf.

35. Austin, L.T., et al., Breast and cervical cancer screening in Hispanic women: a literature review using the health belief model. Womens Health Issues, 2002. 12(3): p. 122-8.

36. Coughlin, S.S. and R.J. Uhler, Breast and cervical cancer screening practices among Hispanic women in the United States and Puerto Rico, 1998-1999. Prev Med, 2002. 34(2): p. 242-51.

37. Krupski, T.L., et al., Patterns of care for men with prostate cancer after failure of primary treatment. Cancer, 2006. 107(2): p. 258-65.

38. Hall, S.E., et al., Prostate cancer: socio-economic, geographical and private-health insurance effects on care and survival. BJU Int, 2005. 95(1): p. 51-8.

39. Consedine, N.S., et al., Prostate cancer screening behavior in men from seven ethnic groups: the fear factor. Cancer Epidemiol Biomarkers Prev, 2006. 15(2): p. 228-37.

40. Gany, F.M., et al., Attitudes, knowledge, and health-seeking behaviors of five immigrant minority communities in the prevention and screening of cancer: a focus group approach. Ethn Health, 2006. 11(1): p. 19-39.

41. Goel, M.S., et al., Racial and ethnic disparities in cancer screening: the importance of foreign birth as a barrier to care. J Gen Intern Med, 2003. 18(12): p. 1028-35.

42. Kobetz, E., et al., Community-based participatory research in Little Haiti: challenges and lessons learned. Prog Community Health Partnersh, 2009. 3(2): p. 133-7.

43. Smith, R.A., et al., Cancer screening in the United States, 2011: A review of current American Cancer Society guidelines and issues in cancer screening. CA Cancer J Clin, 2011. 61(1): p. 8-30.

44. Mastalski, K., et al., Substantial family history of prostate cancer in black men recruited for prostate cancer screening: results from the Prostate Cancer Risk Assessment Program. Cancer, 2008. 113(9): p. 2559-64.

45. Cotter, M.P., et al., Role of family history and ethnicity on the mode and age of prostate cancer presentation. Prostate, 2002. 50(4): p. 216-21.

46. Sharma, S., et al., Assessing dietary patterns in Barbados highlights the need for nutritional intervention to reduce risk of chronic disease. J Hum Nutr Diet, 2008. 21(2): p. $150-8$. 
47. Watters, J.L., et al., Cigarette smoking and prostate cancer in a prospective US cohort study. Cancer Epidemiol Biomarkers Prev, 2009. 18(9): p. 2427-35.

48. de Kok, I.M., et al., Childhood social class and cancer incidence: results of the globe study. Soc Sci Med, 2008. 66(5): p. 1131-9.

49. Franceschi, S., et al., Differences in the risk of cervical cancer and human papillomavirus infection by education level. Br J Cancer, 2009. 101(5): p. 865-70.

50. Brown, N., et al., Assessment of preventive health knowledge and behaviors of AfricanAmerican and Afro-Caribbean women in urban settings. J Natl Med Assoc, 2006. 98(10): p. 1644-51.

51. Tsu, V.D. and C.E. Levin, Making the case for cervical cancer prevention: what about equity? Reprod Health Matters, 2008. 16(32): p. 104-12.

52. DeChello, L.M., D.I. Gregorio, and H. Samociuk, Race-specific geography of prostate cancer incidence. Int J Health Geogr, 2006. 5: p. 59.

53. MacKinnon, J.A., et al., Detecting an association between socioeconomic status and late stage breast cancer using spatial analysis and area-based measures. Cancer Epidemiol Biomarkers Prev, 2007. 16(4): p. 756-62.

54. NCI, What You Need to Know About Prostate Cancer, D.o.H.a.H. Services, Editor. 2005, National Cancer Institute.

55. Chinegwundoh, F., et al., Risk and presenting features of prostate cancer amongst African-Caribbean, South Asian and European men in North-east London. BJU Int, 2006. 98(6): p. 1216-20.

56. Centers for Disease Control, National Health Interview Survey, U.S.D.o.H.a.H. Services, Editor. 2007: Hyattsville.

57. Botman, S.L., et al., Design and Estimation for the Natinal Health Interview Survey, 1995 - 2004. National Center for Health Statistics, 2000. Vital Health Stat 2(130).

58. United States. Central Intelligence Agency. The world factbook.

59. National Center for Health Statistics, Variance Estimation and Other Analytic Issues, NHIS 2006-2009. 2010, Centers for Disease Control and Prevention.

60. Muthen, L.K. and B.O. Muthen, Mplus user's guide: Statistical analysis with latent variables. 5th ed. 1998-2007, Los Angeles: Muthen \& Muthen.

61. Hair, J.F., Multivariate data analysis. 6th ed. 2006, Upper Saddle River, N.J.: Pearson Prentice Hall. xxiv, 899 p.

62. Kline, R., Principles and practice of structural equation modeling. 2nd ed. 2005, New York, NY: Guilford Press. 
63. Day JC, Population Projections of the United States vy Age, Sex, Race, and Hispanic Origin: 1995 - 2050, in Current Population Reports, G.P. Office, Editor. 1996, U.S. Bureau of the Census: Washington DC.

64. Kahn, H.A. and C.T. Sempos, Statistical methods in epidemiology. Monographs in epidemiology and biostatistics. 1989, New York: Oxford University Press. xiii, 292 p.

65. Gordis, L., Epidemiology. 2nd ed. 2000, Philadelphia: W.B. Saunders. xii, 308 p.

66. Kleinbaum, D.G., Applied regression analysis and other multivariable methods. 4th ed. 2007, Australia ; Belmont, CA: Brooks/Cole. xxi, 906 p.

67. Kline, R.B., Principles annd practices of structural equation modeling. 2nd ed. 2005, New York: The guilford Press.

68. Beran, T.N. and C. Violato, Structural equation modeling in medical research: a primer. BMC Res Notes. 3: p. 267.

69. Allison, P., Estimation of Linear Models with Incomplete Data. Sociological Methodology, 1987. 17: p. 71-103.

70. Liu, X., M.M. Wall, and J.S. Hodges, Generalized spatial structural equation models. Biostatistics, 2005. 6(4): p. 539-57.

71. Klein RJ, et al., Healthy People 2010 Criteria for Data Suppression, in Statistical Notes. June 2002, National Center for Health Statistics: Hyatsville, MD.

72. Schermelleh-Engel K and M. H, Evaluating the Fit of Structural Equation Models: Tests of Significance and Descriptive Goodness-of-Fit Measures. Methods of Psychological Research Online, 2003. 8(2): p. 23-74.

73. U.S. Census Bureau. American Community Survey. 2010 March 2, 2011; 8/22/2009:[Available from: http://factfinder.census.gov/servlet/DatasetMainPageServlet? program=DEC\& submenu $\underline{\mathrm{Id}=\& \text { lang }=\text { en \& } \mathrm{ts}=.}$

74. Mohanty, S.A., et al., Health care expenditures of immigrants in the United States: a nationally representative analysis. Am J Public Health, 2005. 95(8): p. 1431-8.

75. Derose, K.P., J.J. Escarce, and N. Lurie, Immigrants and health care: sources of vulnerability. Health Aff (Millwood), 2007. 26(5): p. 1258-68.

76. Okie, S., Immigrants and health care--at the intersection of two broken systems. N Engl J Med, 2007. 357(6): p. 525-9.

77. Drake, B.F., et al., Racial differences in prostate cancer screening by family history. Ann Epidemiol, 2008. 18(7): p. 579-83. 
78. Meiser, B., et al., Prostate cancer screening in men with a family history of prostate cancer: the role of partners in influencing men's screening uptake. Urology, 2007. 70(4): p. $738-42$.

79. Magnus, M., Prostate cancer knowledge among multiethnic black men. J Natl Med Assoc, 2004. 96(5): p. 650-6.

80. Wallner, L.P., et al., Psychosocial factors associated with an increased frequency of prostate cancer screening in men ages 40 to 79 years: the Olmsted County study. Cancer Epidemiol Biomarkers Prev, 2008. 17(12): p. 3588-92.

81. Andriole, G.L., et al., Prostate Cancer Screening in the Prostate, Lung, Colorectal and Ovarian (PLCO) Cancer Screening Trial: findings from the initial screening round of a randomized trial. J Natl Cancer Inst, 2005. 97(6): p. 433-8.

82. Haas, G.P., Sakr, W.A., Epidemiology of Prostate Cancer. CA Cancer J Clin, 1997. 47(5): p. 273-287.

83. Abe, T., I.B. Martin, and L.M. Roche, Clusters of census tracts with high proportions of men with distant-stage prostate cancer incidence in New Jersey, 1995 to 1999. Am J Prev Med, 2006. 30(2 Suppl): p. S60-6.

84. Coard, K.C. and D.H. Skeete, A 6-year analysis of the clinicopathological profile of patients with prostate cancer at the University Hospital of the West Indies, Jamaica. BJU Int, 2009. 103(11): p. 1482-6.

85. Gilligan, T., et al., Racial differences in screening for prostate cancer in the elderly. Arch Intern Med, 2004. 164(17): p. 1858-64.

86. Garbers, S. and M.A. Chiasson, Breast cancer screening and health behaviors among African American and Caribbean Women in New York City. J Health Care Poor Underserved, 2006. 17(1): p. 37-46.

87. World Health Organization. Prevalence of tobacco use among adults and adolescents. Prevalence of smoking any tobacco product among adults aged 15+years, 2006: Male 2006 [cited 2011 April 3, 2011]; Available from:

http://gamapserver.who.int/gho/interactive_charts/tobacco/use/atlas.html.

88. Osypuk, T.L. and D. Acevedo-Garcia, Support for smoke-free policies: a nationwide analysis of immigrants, US-born, and other demographic groups, 1995-2002. Am J Public Health, 2009. 100(1): p. 171-81.

89. Gallus, S., et al., Temporal changes of under-reporting of cigarette consumption in population-based studies. Tob Control, 2011. 20(1): p. 34-9.

90. Chen, J. and A. Vargas-Bustamante, Estimating the Effects of Immigration Status on Mental Health Care Utilizations in the United States. J Immigr Minor Health, 2011. 
91. Carrasquillo, O., A.I. Carrasquillo, and S. Shea, Health insurance coverage of immigrants living in the United States: differences by citizenship status and country of origin. Am J Public Health, 2000. 90(6): p. 917-23.

92. Arthur, C.M. and E.S. Katkin, Making a case for the examination of ethnicity of Blacks in United States Health Research. J Health Care Poor Underserved, 2006. 17(1): p. 25-36. 


\section{APPENDICES}

Table 1a. Summary of STATA commands

\begin{tabular}{|l|l|}
\hline set mem 200m & $\begin{array}{l}\text { set memory allows you to increase or decrease the amount of memory } \\
\text { allocated to Stata by the operating system while Stata is running. }\end{array}$ \\
\hline svyset psu_p & $\begin{array}{l}\text { svyset declares the data to be complex survey data, designates variables } \\
\text { that contain information about the survey design, and specifies the } \\
\text { default method for variance estimation. } \\
\text { pweights, or sampling weights, are weights that denote the inverse of the } \\
\text { probability that the observation is included because of the sampling } \\
\text { design. }\end{array}$ \\
strata(strat_p) & $\begin{array}{l}\text { strata }] \\
\text { strata specifies the name of a variable (numeric or string) that contains } \\
\text { stratum identifiers. }\end{array}$ \\
\hline svy:tab & $\begin{array}{l}\text { Produces two-way tables of frequency counts, and proportions, along } \\
\text { with various measures of association, including the common Pearson's } \\
\text { chi-squared and the likelihood-ratio chi-squared. }\end{array}$ \\
\hline svy:logistic & $\begin{array}{l}\text { Fits a logistic regression model of the dependent variable, where the } \\
\text { dependent variable is a 0/1 variable. Provides odds ratios }\end{array}$ \\
\hline
\end{tabular}


Statistical analysis for Aim 2 was conducted using a combination of STATA 10 software and Mplus software. STATA was used to prepare the data and Mplus was used to conduct the analysis.

Table 2a. Mplus data analysis codes.

\begin{tabular}{cl}
\hline TITLE & \\
\hline VARIABLE & $\begin{array}{l}\text { The variable command provides information about the variables in } \\
\text { the data set to be analyzed }\end{array}$ \\
\hline NAMES ARE & The NAMES option assigns names to the variables in the data set \\
\hline MISSING ARE ALL & $\begin{array}{l}\text { Identifies the values in the analysis that are to be treated as missing } \\
\text { or invalid. }\end{array}$ \\
\hline USEVARIABLES & This option is used to select a subset of variables for analysis \\
\hline CATEGORICAL ARE & $\begin{array}{l}\text { Specifies which dependent variables are treated as binary or } \\
\text { ordered categorical variables in the model and its estimation }\end{array}$
\end{tabular}

\section{ANALYSIS:}

ESTIMATOR=MLR; $\quad$ Maximum likelihood estimation with robust standard errors

\begin{tabular}{cl}
\hline TYPE & Describes the type of analysis to be performed \\
\hline GENERAL & $\begin{array}{l}\text { Default option that allows analysis of relationships among } \\
\text { observed and continuous latent variables, observed variables, and } \\
\text { among continuous latent variables. }\end{array}$ \\
\hline MODEL & This command describes the model to be estimated. \\
\hline WITH & $\begin{array}{l}\text { Describes the linear regression of the dependent variable on the } \\
\text { covariate independent variables. }\end{array}$ \\
\hline & $\begin{array}{l}\text { Residual covariance between covariates is fixed at zero and this } \\
\text { statement frees this parameter and allows for assessment of } \\
\text { covariance. }\end{array}$ \\
\hline
\end{tabular}

Used to request additional output not included as the default.

\section{OUTPUT:}

Standardized PATTERNS; This option provides a summary of missing data patterns 
VITA

NICOLE A. KELLIER

Born, Miami, Florida

1999

B.A., Interdisciplinary Natural Sciences

University of South Florida

Tampa, Florida

$2000-2001$

Quality Assurance Associate

Specialized Nursing Services

Miami, Florida

$2002-2003$

Research Assistant

George Washington University

Washington, District of Columbia

MPH, Epidemiology

George Washington University

Washington, District of Columbia

$2003-2005$

Research Associate II/III

University of Miami

Miami, Florida

$2005-2006$

Assistant Administrator

Karing Nursing Registry

Boca Raton, Florida

$2006-2010$

Teaching Assistant/Research Assistant

Florida International University

Miami, Florida

2010

Research Scientist

Eli Lilly and Company

Indianapolis, Indiana

\section{PUBLICATIONS AND PRESENTATIONS}

Brinks A, Kellier N. Stress, social support and health locus of control as predictors of psychological distress in HIV-infected women. 2007. Presented at The Annual Public Health Association (APHA) Conference. Washington DC. November 2-7, 2007.

Kellier N, et al. Baseline Assessment of Cardiovascular Risk among Typical and Atypical Antipsychotic Drug Users. Presented at Lilly Expo February 28, 2010. 
Kellier N, Niyonsenga T. Low Birth Weight Rates in two Counties of South Florida: Small-Area Variation and Temporal Trends. 2007 American College of Epidemiology (ACE) Meeting, Presented in Ft. Lauderdale, Florida. September 15-18, 2007 